\title{
General $U(1) \times U(1)$ F-theory compactifications and beyond: geometry of unHiggsings and novel matter structure
}

\author{
Mirjam Cvetič, ${ }^{a, b}$ Denis Klevers, ${ }^{c}$ Hernan Piragua ${ }^{a}$ and Washington Taylor ${ }^{d}$ \\ ${ }^{a}$ Department of Physics and Astronomy, University of Pennsylvania, \\ 209 S. 33rd Street, Philadelphia, PA 19104-6396, U.S.A. \\ ${ }^{b}$ Center for Applied Mathematics and Theoretical Physics, University of Maribor, \\ Krekova Ulica 2, 2000 Maribor, Slovenia \\ ${ }^{c}$ Theory Group, Physics Department, CERN, \\ CH-1211, Geneva 23, Switzerland \\ ${ }^{d}$ Center for Theoretical Physics, Department of Physics, Massachusetts Institute of Technology, \\ 77 Massachusetts Avenue Cambridge, MA 02139, U.S.A. \\ E-mail: cvetic@cvetic.hep.upenn.edu, denis.klevers@cern.ch, \\ hpiragua@sas.upenn.edu, wati@mit.edu
}

ABSTRACT: We construct the general form of an F-theory compactification with two U(1) factors based on a general elliptically fibered Calabi-Yau manifold with Mordell-Weil group of rank two. This construction produces broad classes of models with diverse matter spectra, including many that are not realized in earlier F-theory constructions with $\mathrm{U}(1) \times \mathrm{U}(1)$ gauge symmetry. Generic $\mathrm{U}(1) \times \mathrm{U}(1)$ models can be related to a Higgsed non-Abelian model with gauge group $\mathrm{SU}(2) \times \mathrm{SU}(2) \times \mathrm{SU}(3), \mathrm{SU}(2)^{3} \times \mathrm{SU}(3)$, or a subgroup thereof. The nonlocal horizontal divisors of the Mordell-Weil group are replaced with local vertical divisors associated with the Cartan generators of non-Abelian gauge groups from Kodaira singularities. We give a global resolution of codimension two singularities of the Abelian model; we identify the full anomaly free matter content, and match it to the unHiggsed non-Abelian model. The non-Abelian Weierstrass model exhibits a new algebraic description of the singularities in the fibration that results in the first explicit construction of matter in the symmetric representation of $\mathrm{SU}(3)$. This matter is realized on double point singularities of the discriminant locus. The construction suggests a generalization to $\mathrm{U}(1)^{k}$ factors with $k>2$, which can be studied by Higgsing theories with larger non-Abelian gauge groups.

Keywords: F-Theory, Differential and Algebraic Geometry, Gauge Symmetry

ArXiv EPRINT: 1507.05954 


\section{Contents}

1 Introduction 1

$2 \quad \mathrm{U}(1)$ 's and unHiggsing $\quad 4$

2.1 Higgsing and a single U(1) 4

2.1.1 Higgsing an $\mathrm{SU}(2) \quad 4$

2.1.2 UnHiggsing a U(1) 5

2.2 Higgsing and two U(1)'s: simple constructions 6

2.2.1 Higgsing $\mathrm{SU}(2) \times \mathrm{SU}(2) \quad 7$

2.2.2 Higgsing $\mathrm{SU}(3) \quad 7$

2.2.3 Hybrid models from $\mathrm{SU}(2) \times \mathrm{SU}(2) \times \mathrm{SU}(3) \quad 9$

$\begin{array}{ll}2.2 .4 \text { Singular divisors and higher charges } & 10\end{array}$

$\begin{array}{lll}2.3 & \text { Examples } & 12\end{array}$

3 General elliptic fibrations with three sections $\quad 13$

$\begin{array}{ll}3.1 \text { A new elliptic curve } & 13\end{array}$

$\begin{array}{lll}3.1 .1 & \text { Specialized models } & 15\end{array}$

$\begin{array}{lll}3.2 & \text { The Weierstrass form } & 16\end{array}$

$\begin{array}{lll}3.3 & \text { Constructing elliptic fibrations } & 17\end{array}$

$\begin{array}{ll}3.4 & \text { Comparison with } d P_{2} \text {-elliptic fibrations } \\ \end{array}$

4 Singularities, global resolution and the matter spectrum 20

$\begin{array}{ll}4.1 \text { Singularities of } X_{n+1} \text { and their global resolution } & 21\end{array}$

4.1.1 Singularities in the cubic fibration $X_{n+1} \quad 22$

4.1.2 Global resolution of the cubic fibration $X_{n+1} \quad 23$

$\begin{array}{lll}4.2 & \text { The matter spectrum } & 25\end{array}$

$\begin{array}{lll}4.2 .1 & \text { General model } & 25\end{array}$

4.2.2 Specialized model 28

$\begin{array}{lll}4.3 & \text { Comparison with matter in } d P_{2} \text {-elliptic fibrations } & 29\end{array}$

5 UnHigssing two U(1)'s in F-theory $\quad 30$

5.1 UnHiggsing in the complex structure moduli space 31

5.2 Geometrical unHiggsing of one of the U(1)'s 32

5.3 Unhiggsing both U(1)'s 34

5.4 Weierstrass model from unHiggsing the general $\mathrm{U}(1)^{2}$ model 37

$\begin{array}{ll}5.5 & \text { The matter spectrum } \\ 5.6 & 38\end{array}$

5.6 Special unHiggsings: $b_{i} \rightarrow 0 \quad 41$

5.7 Novel Weierstrass models with $I_{3}^{\text {s }}$ singularities 43 
6 UnHiggsing of two U(1)'s: concrete examples 44

6.1 UnHiggsing of two U(1)'s to $\mathrm{SU}(2) \times \mathrm{SU}(2) \quad 45$

6.1.1 Geometric discussion $\quad 46$

6.1.2 UnHiggsing from matching of spectra 47

6.1.3 Examples: models on $\mathbb{P}^{2} \quad 47$

6.2 UnHiggsings of two U(1)'s to SU(3) 48

6.2.1 Geometric discussion 48

6.2.2 UnHiggsing from matching of spectra $\quad 50$

6.2.3 Examples: models on $\mathbb{P}^{2} \quad 50$

6.3 Unhiggsing of two $\mathrm{U}(1)$ 's to $\mathrm{SU}(2) \times \mathrm{SU}(2) \times \mathrm{SU}(3)$

6.3.1 Geometric discussion \& comparison to field theory 52

6.3.2 Examples: models on $\mathbb{P}^{2} \quad 56$

$\begin{array}{lll}7 & \text { Further research directions } & \mathbf{5 8}\end{array}$

$\begin{array}{lll}7.1 & \text { More U(1) factors } & 58\end{array}$

$\begin{array}{lll}7.2 & \text { Exotic matter representations } & 60\end{array}$

$\begin{array}{lll}7.3 & \text { Matching 6D supergravity } & 61\end{array}$

8 Conclusions $\quad 62$

A The Weierstrass form of $X_{n+1} \quad 64$

B Weierstrass models with $I_{2}$ and $I_{3}$ singularities $\quad 66$

B.1 The $\mathrm{SU}(2) \times \mathrm{SU}(2)$ Weierstrass model $\quad 67$

B.2 The $I_{3}$ Weierstrass model 68

$\begin{array}{lll}\text { B.3 The } \mathrm{SU}(2) \times \mathrm{SU}(2) \times \mathrm{SU}(3) \text { Weierstrass model } & 70\end{array}$

C Map of $X_{n+1}$ to the quartic in $\mathrm{Bl}_{1} \mathbb{P}^{2}(1,1,2) \quad 71$

\section{Introduction}

F-theory [1-3] provides a powerful nonperturbative approach to understanding large classes of string vacua in four and six space-time dimensions. While non-Abelian gauge factors in F-theory models are classified by the local Kodaira-Tate classification of singular fibers in elliptic fibrations, Abelian factors are represented by elements of the Mordell-Weil group, which are intrinsically global and more difficult to describe analytically. In recent years, progress has been made on constructing general classes of Weierstrass models that describe F-theory compactifications with one or more Abelian U(1) factors. The general form of an F-theory Weierstrass model with a single U(1) factor was described by Morrison and Park in [4]. ${ }^{1}$ A general class of models with two U(1) factors was constructed in [8-12], and models with three $\mathrm{U}(1)$ factors were studied in [13].

\footnotetext{
${ }^{1}$ Certain F-theory compactifications with U(1) factors have appeared earlier in $[5,6]$ in the context of heterotic/F-theory duality, see also [7].
} 
Explicit constructions of general F-theory models with multiple U(1) factors are algebraically quite complex. A great deal of physical insight can be gained into systems with Abelian factors by considering how the Abelian factors can arise from Higgsing of non-Abelian gauge factors. For example, a model with a single U(1) can be constructed by starting with an $\mathrm{SU}(2)$ non-Abelian factor tuned on a divisor that supports an adjoint representation, and then Higgsing the $\mathrm{SU}(2)$ by giving a vacuum expectation value to the adjoint matter, giving a breaking $\mathrm{SU}(2) \rightarrow \mathrm{U}(1)$. A similar consideration allows us to construct a fairly broad class of models with gauge group $\mathrm{U}(1) \times \mathrm{U}(1)$ by Higgsing a rank two gauge group, such as $\mathrm{SU}(2) \times \mathrm{SU}(2)$ or $\mathrm{SU}(3)$. Consideration of the resulting spectra, however, shows that the $\mathrm{U}(1) \times \mathrm{U}(1)$ models constructed in this way cannot in general be described through the construction of [8-12]..$^{2}$ This observation motivates us to find a more general approach to constructing the F-theory models with two U(1) factors.

The main result of this paper is the construction of a very general Weierstrass form for F-theory models with $\mathrm{U}(1) \times \mathrm{U}(1)$ gauge factors. This class of models includes all those that can be realized by Higgsing the rank two groups $\mathrm{SU}(2) \times \mathrm{SU}(2)$ and $\mathrm{SU}(3)$ on adjoint matter. The most general model also contains more complex spectra, which can arise from Higgsing more complicated non-Abelian structures. In fact, one of the principal results of this paper is that a large class of generic models with two U(1) factors can be "unHiggsed" to models with the non-Abelian gauge group $G_{\text {general }}=\mathrm{SU}(2) \times \mathrm{SU}(2) \times \mathrm{SU}(3)$. In various special cases, the model is unHiggsed to a subgroup of this group, $G \subset G_{\text {general }}$. In this unHiggsing process, the "horizontal" divisors associated with sections in the Mordell-Weil group become vertical divisors associated with Kodaira singularity types in the elliptic fibration over the base. This generalizes the result found in $[4,14]$ that a single $\mathrm{U}(1)$ factor can generally be unHiggsed to an $\mathrm{SU}(2)$ non-Abelian factor (or an increase in rank on an existing non-Abelian factor), though in some cases the resulting non-Abelian model may have certain types of singularities. The form of the non-Abelian group $G_{\text {general }}$ can be understood geometrically by identifying the general form of the vertical divisors associated with unHiggsing the two $\mathrm{U}(1)$ factors to be $A C, B C$, where $C$ is a common factor. The unHiggsing process leads to $\mathrm{SU}(2)$ factors on $A$ and $B$, while on $C$ the two elements of the Cartan generators combine in a nontrivial way to produce the group $\mathrm{SU}(3)$. In some cases the divisors $A, B$ and $C$ can or must be reducible, thus leading to larger non-Abelian groups such as $\mathrm{SU}(2)^{3} \times \mathrm{SU}(3)$. This general algebraic framework suggests an approach to describing Weierstrass models with more Abelian factors in terms of analogous, but more complicated, algebraic structures.

A systematic understanding and classification of F-theory models with multiple Abelian factors is an important challenge for F-theory, both for theoretical and phenomenological reasons. Much recent work has focused on various aspects of this problem, largely motivated by efforts to construct models with Abelian factors related to F-theory GUT phenomenology (for a representative list of works, see $[10,11,15-30]$ ) as well as other particle physics models with Abelian gauge symmetry [31-33]. On the more theoretical

\footnotetext{
${ }^{2}$ On $\mathbb{P}^{2}$, for example, only an $\mathrm{SU}(3)$ model with one adjoint and 54 fundamentals Higgses to such a $\mathrm{U}(1) \times \mathrm{U}(1)$ model.
} 
side, a major challenge in constructing a completely general F-theory model with Abelian factors is the wide range of possible spectra that may arise in such a theory. While for $6 \mathrm{D}$ models anomaly constraints in the low-energy supergravity theory provide some limits on the set of possibilities [34-37], as one considers matter with increasingly large charges under the $\mathrm{U}(1)$ gauge factors, the complexity of the corresponding F-theory models grows accordingly. Through the unHiggsing process, these matter fields with higher charges are related to matter fields with increasingly complicated transformation properties under the corresponding non-Abelian gauge factors.

One of the important new results of this paper that signifies the complexity of the representations with multiple $\mathrm{U}(1)$ factors is a construction of a generic class of $\mathrm{U}(1) \times \mathrm{U}(1)$ models in which the resolution of the Abelian theory gives matter fields with specific higher charges that are related to matter transforming under the symmetric representation of $\mathrm{SU}(3)$ in the associated unHiggsed theory. As a by-product this is the first explicit realization of the the symmetric matter representation of the non-Abelian gauge symmetry in F-theory. The associated Weierstrass models have an intricate and nontrivial algebraic structure that realizes the non-Abelian gauge theory in a novel way that cannot be understood directly from the Tate description.

While our construction and the geometric results are applicable for Calabi-Yau nfolds, in this paper we primarily focus on Calabi-Yau threefolds relevant for 6D F-theory. However, most of the geometric analyses are also relevant to 4D F-theory.

The outline of this paper is as follows: in section 2, we discuss the ways in which models with one or two U(1) factors can be realized by Higgsing non-Abelian gauge groups with a variety of structures. This field-theoretic analysis and discussion provides a framework for interpreting the rather complicated algebraic structures that arise in the following sections. In section 3, we construct the most general elliptic fibration with three independent sections, corresponding to a Weierstrass model with Mordell-Weil group of rank two, and an F-theory model having two Abelian gauge factors $\mathrm{U}(1) \times \mathrm{U}(1)$ in the corresponding low-energy supergravity theory. In section 4 , we give a global resolution of the singularities in the general $\mathrm{U}(1) \times \mathrm{U}(1)$ Weierstrass model, and analyze the resulting matter spectrum. In section 5, we describe in general terms the unHiggsing of the two $\mathrm{U}(1)$ model to a non-Abelian theory with vanishing rank of Mordell-Weil group. Concrete examples of the unHiggsing process and the detailed form of the Weierstrass model in different cases are explored in section 6 . In section 7 , we discuss a natural generalization of our work to more than two U(1) factors and the construction of F-theory models with exotic matter representations as well as the problem of proving the global equivalence of the space of consistent $6 \mathrm{D}$ supergravity theories and the set of F-theory compactifications. We summarize key results of the paper in our conclusions in section 8 .

Note added. Our general $\mathrm{U}(1) \times \mathrm{U}(1)$ F-theory construction, presented in this paper, is already employed in the simultaneous work [38] for the study of flavor textures in SU(5) GUT's with two U(1)'s. 


\section{$2 \quad \mathrm{U}(1)$ 's and unHiggsing}

In this section we describe various ways that two U(1)'s can arise from the Higgsing of a non-Abelian theory. This provides a field theory framework for understanding the wide range of models that can be produced using the general $\mathrm{U}(1) \times \mathrm{U}(1)$ Weierstrass model constructed in the following section. We begin with a review of the story for a single $\mathrm{U}(1)$, and then consider unHiggsing of two $\mathrm{U}(1)$ factors.

The general classes of models considered in this section can be constructed in the context of F-theory in six or four space-time dimensions. For specific examples, we focus here on $6 \mathrm{D}$ constructions, since anomaly cancellation conditions for $6 \mathrm{D}$ supergravity theories provide strong constraints on the allowed spectra of the low-energy theories [35, 39, 40], providing in many cases a simple check on the consistency of the constructions we describe here. The same classes of theories can also be realized in 4D F-theory constructions, however, with a richer range of specific models and applications.

\subsection{Higgsing and a single $\mathrm{U}(1)$}

\subsubsection{Higgsing an $\mathrm{SU}(2)$}

In general, an $\mathrm{SU}(2)$ gauge factor with matter in the adjoint representation can be constructed in F-theory by tuning a Kodaira type $I_{2}$ singularity on a divisor $D$ of the form $-K_{B}+X$, where $K_{B}$ is the canonical class of the F-theory base manifold $B_{n}$, and $X$ is effective; for such $D$, we write $D \geq-K_{B}$. For a smooth divisor $D$, this condition is necessary and sufficient for the existence of an adjoint matter representation at the level of geometry. For $6 \mathrm{D}$ theories, this can be seen directly from the fact that the genus $g$ of the divisor $D$ is in general given by

$$
g=1+\frac{1}{2} D \cdot\left(D+K_{B}\right),
$$

which is positive precisely for $D \geq-K_{B}$. As a simple example, if the base is $B_{2}=\mathbb{P}^{2}$, then $-K_{B}=3 H_{B}$ where $H_{B}$ is the hyperplane class in $\mathbb{P}^{2}$. If we tune an $\mathrm{SU}(2)$ over a smooth curve of degree $d$ and genus $g=(d-1)(d-2) / 2$, in the resulting low-energy theory there are $g$ matter fields that transform in the adjoint of $\mathrm{SU}(2)$. For example, a cubic curve $C$ has genus 1, a quartic has genus 3, etc. . From anomaly cancellation or inspection of the explicit Weierstrass model, cf. appendix B.1, the number of fundamental matter fields is $x_{2}=6 d^{2}+16(1-g)$. For the cubic curve $C$, this gives $x_{2}=54$.

An SU(2) with adjoint matter can be Higgsed by giving a VEV to an adjoint matter field proportional to the Cartan generator $\sigma_{3}$, the third Pauli matrix. This leaves a residual $\mathrm{U}(1)$ gauge symmetry. Each matter field that transforms in the fundamental of the original $\mathrm{SU}(2)$ gives rise to a pair of matter fields with charges \pm 1 under the resulting $\mathrm{U}(1)$. For example, if we start with a cubic on $\mathbb{P}^{2}$, after Higgsing we get a $U(1)$ theory with 108 charged matter fields - the minimum number of charged matter fields compatible with anomaly cancellation in a $6 \mathrm{D}$ supergravity theory with a single $\mathrm{U}(1)$ factor [37]. More generally, if we Higgs the $\mathrm{SU}(2)$ using one adjoint (whose charged components are eaten up) and there are also $g-1$ other adjoint fields available, each of the $g-1$ other adjoints 
produces a multiplet of charges $(-2,0,+2)$ under the resulting $\mathrm{U}(1)$. For example, tuning an $\mathrm{SU}(2)$ on a quartic on $\mathbb{P}^{2}$ and then Higgsing gives 128 matter fields with charges \pm 1 and 4 matter fields with charges \pm 2 .

\subsubsection{UnHiggsing a $\mathrm{U}(1)$}

An F-theory compactification on a base manifold $B$ is defined through a Weierstrass model $y^{2}=x^{3}+f x z^{4}+g z^{6}$, where $f$ and $g$ are sections $f \in \Gamma\left(\mathcal{O}\left(-4 K_{B}\right)\right), g \in \Gamma\left(\mathcal{O}\left(-6 K_{B}\right)\right)$. Such an elliptic fibration has a global section $z=0$. We often work in the coordinate patch of $\mathbb{P}(2,3,1)$ where $z=1$, giving the common form

$$
y^{2}=x^{3}+f x+g
$$

It was shown in [4], using the elliptic curve in $\mathrm{Bl}_{1} \mathbb{P}^{2}(1,1,2)$, that the general form of a Weierstrass model with a $\mathrm{U}(1)$ factor takes the form

$$
y^{2}=x^{3}+\left(e_{1} e_{3}-\frac{1}{3} e_{2}^{2}-b^{2} e_{0}\right) x+\left(-e_{0} e_{3}^{2}+\frac{1}{3} e_{1} e_{2} e_{3}-\frac{2}{27} e_{2}^{3}+\frac{2}{3} b^{2} e_{0} e_{2}-\frac{1}{4} b^{2} e_{1}^{2}\right) .
$$

Here, $b$ is a section of a line bundle $\mathcal{O}(L)$, where $L$ is effective, and $e_{i}$ are sections of line bundles $\mathcal{O}\left((i-4) K_{B}+(i-2) L\right)$. The Weierstrass model (2.3) has a nontrivial rational section, so that the Mordell-Weil group of rational sections has rank (at least) 1. As described in $[4,14]$, when the parameter $b$ is taken to vanish, the divisor associated to this rational section is transformed to a vertical divisor. In general, assuming that in the original $\mathrm{U}(1)$ model there is no Kodaira singularity on the divisor ${ }^{3} e_{3}$, this leads to a non-Abelian $\mathrm{SU}(2)$ factor on $e_{3}$, giving an unHiggsing corresponding to the reverse of the process described above. Note that ${ }^{4}\left[e_{3}\right]=-K_{B}+L$ always has a form that allows for an adjoint representation of the resulting $\mathrm{SU}(2)$.

In situations where the original model (2.3) already has some non-Abelian gauge group components, the story can be slightly more complicated. In some cases, tuning the $\mathrm{SU}(2)$ can lead to $(4,6)$ singularities at codimension one or two. If the divisor $\left[e_{3}\right]$ already itself supports a non-Abelian gauge factor, then tuning $b \rightarrow 0$ leads to an enhancement of the original gauge factor with an increase in rank, as the horizontal divisor of the rational section is transformed into vertical form. The form of (2.3) shows that any $\mathrm{U}(1)$ can thus be "unHiggsed" corresponding to an enhancement of the non-Abelian sector of the theory, albeit in some cases with resulting singularities.

Another relevant situation can occur when $e_{3}=\alpha \beta$, i.e. $e_{3}$ is reducible. In this case, performing the unHiggsing by taking $b \rightarrow 0$ leads to an $\mathrm{SU}(2)$ factor on each component of $e_{3}$. For example, consider the case where the base is $B=\mathbb{P}^{2}$ and $\left[e_{3}\right]=3 H,[\alpha]=H,[\beta]=$ $2 H$. In this case, the unHiggsing gives a theory with $\mathrm{SU}(2)$ factors on a pair of intersecting curves $\alpha, \beta$ of degrees one and two. In this case there is no adjoint matter field to Higgs. The spectrum of the resulting theory in this example consists of 22 fundamentals on $\alpha$ and 40 fundamentals on $\beta$, including two bifundamental hypermultiplets $(\mathbf{2}, \mathbf{2})$ associated with

\footnotetext{
${ }^{3}$ As a short-hand notation, we denote here and in the following e.g. the divisor $e_{3}=0$ simply by $e_{3}$.

${ }^{4}$ We will denote the divisor class of a section on $B_{n}$ with brackets, e.g. the class of $e_{3}$ by $\left[e_{3}\right]$.
} 
the two intersection points, cf. the general multiplicity formulae (B.9). The bifundamental fields can be Higgsed by turning on VEV's of the form $\operatorname{diag}\left(v_{1}, v_{2}\right)$ for two independent VEV's $v_{1}, v_{2}$. Note that two fields must be used for this Higgsing to satisfy the D-flatness conditions when fields in the fundamental representation are used, unlike in the case of Higgsing on an adjoint field where only one field is needed since the D-term constraints are automatically satisfied. Each bifundamental contributes two fundamentals to the spectrum on each curve, so after the Higgsing there are $18+36=54 \mathrm{SU}(2)$ fundamentals that are broken to pairs of \pm 1 charges under the resulting $\mathrm{U}(1)$, reproducing the expected number 108 of charged hypers. This kind of model can be understood as simply a degeneration of a single divisor $e_{3}$ to a combination of divisors $\alpha \beta$. The Higgsing can be thought of as occurring in two stages: first a Higgsing by a VEV proportional to e.g. $\mathbb{1}$ that re-combines the two components, and then a Higgsing by the resulting adjoint. After the complete Higgsing, the remaining $\mathrm{U}(1)$ can be seen as the difference $\sigma_{3}^{(1)}-\sigma_{3}^{(2)}$ of Cartan generators of the original $\mathrm{SU}(2)$ factors.

Note that in the case where $e_{3}$ is reducible, if either factor $\alpha, \beta$ can by itself support an adjoint, i.e., if $\alpha \geq-K_{B}$ or $\beta \geq-K_{B}$, then the $\mathrm{U}(1)$ model can be seen as coming from an $\mathrm{SU}(2)$ on the divisor $\alpha \beta$ in several different ways. First, in the fashion just described where both $\mathrm{SU}(2)$ 's are Higgsed simultaneously using a pair of bifundamental matter fields, and second by first Higgsing an $\mathrm{SU}(2)$ adjoint to get $\mathrm{U}(1) \times \mathrm{SU}(2)$ and then Higgsing a bifundamental with both $\mathrm{U}(1)$ and $\mathrm{SU}(2)$ charges. For example, if $\alpha \geq-K_{B}$, then the $\mathrm{SU}(2)$ on $\alpha$ already contains an adjoint, and Higgsing that adjoint will produce a theory with gauge group $\mathrm{U}(1) \times \mathrm{SU}(2)$ with the $\mathrm{SU}(2)$ supported on $\beta$. This represents a sort of intermediate stage of the Higgsing process, where the $\mathrm{U}(1)$ in the fully Higgsed model is related to a combination of the $\mathrm{U}(1)$ field associated with $\alpha$ and the Cartan of the $\mathrm{SU}(2)$ on $\beta$. This can be seen algebraically in the F-theory context through the Abelian model (2.3). Here, the residual SU(2) on $\beta$ is introduced by taking $e_{3}=\alpha \beta$ and having a factor of $\beta$ in $b$ as $b=b^{\prime} \beta$. Then, the rational section lives only on $\alpha$ as its coordinates in $\mathrm{Bl}_{1} \mathbb{P}^{2}(1,1,2)$ are $\left[-b^{\prime} \beta: 1: \alpha \beta: 0\right]=\left[-b^{\prime}: 1: \alpha: 0\right]$ employing the $\mathbb{C}^{*}$-action. Note also that in the fully unHiggsed model with $b^{\prime}=0$ the extra factor of $\beta$ can then be moved out of $e_{3}$ by redefining $\tilde{e}_{3}=e_{3} / \beta, \tilde{e}_{1}=\beta e_{1}, \tilde{e}_{0}=\beta^{2} e_{0}$.

We encounter a number of related situations in the later sections of the paper, where various unHiggsed models can be partially Higgsed in various sequences to go between a non-Abelian model and an Abelian model. For the most part, the focus however is on the completely Higgsed and completely unHiggsed endpoints of these processes.

\subsection{Higgsing and two U(1)'s: simple constructions}

Now let us consider some simple ways in which a pair of U(1)'s can be produced from the Higgsing of a non-Abelian model. The simplest way in which this can be done is to start with a theory with a rank two non-Abelian gauge group $\mathrm{SU}(2) \times \mathrm{SU}(2)$ or $\mathrm{SU}(3)$, where each factor has an adjoint representation that can be Higgsed. One might also consider a theory with group $G_{2}$, but a Higgsing of $G_{2}$ on the adjoint can give $\mathrm{SU}(3)$, so the resulting $\mathrm{U}(1) \times \mathrm{U}(1)$ models and spectra are identical to those that could come from an original $\mathrm{SU}(3)$ model. 


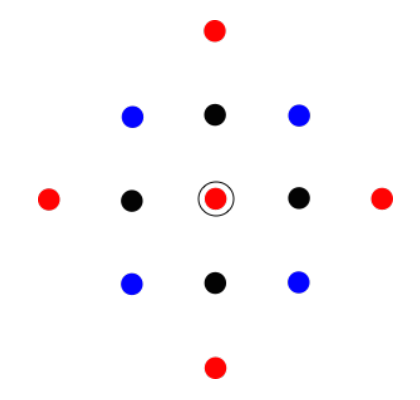

Figure 1. Weight lattice of charges in a theory with a $\mathrm{U}(1) \times \mathrm{U}(1)$ gauge group realized by Higgsing a theory with gauge group $\mathrm{SU}(2) \times \mathrm{SU}(2)$. Dots in red indicate the adjoint, dots in blue the bifundamental and black dots are the fundamentals. The origin is indicated by an extra circle.

\subsubsection{Higgsing $\mathrm{SU}(2) \times \mathrm{SU}(2)$}

If we have two $\mathrm{SU}(2)$ factors that are tuned on two independent smooth divisors $A, B \geq$ $-K_{B}$, then each of the factors carries an adjoint representation, and each can be independently Higgsed just as in the case of a single $\mathrm{SU}(2)$. The resulting model has a gauge group $\mathrm{U}(1) \times \mathrm{U}(1)$. In general, the original model will have some number of fundamentals transforming under each of the $\mathrm{SU}(2)$ factors, and some number of bifundamental fields that transform as a fundamental under each of the factors, in addition to a number of adjoint representations for each group corresponding to the genera of the two curves. After the two factors are Higgsed, the fundamentals will become scalar fields that carry charges $( \pm 1,0)$ and $(0, \pm 1)$ under the two $\mathrm{U}(1)$ factors. The bifundamental fields will carry charges $( \pm 1, \pm 1)$, and the extra adjoint fields will carry nonzero charges $( \pm 2,0),(0, \pm 2)$. This gives a characteristic set of spectra that can arise from this Higgsing structure, depicted in figure 1.

As an example, consider a $6 \mathrm{D}$ model on $\mathbb{P}^{2}$. If we tune an $\mathrm{SU}(2)$ on each of two divisors $A, B$ of degrees $a, b \geq 3$, then we will have $g_{a}=(a-1)(a-2) / 2$ adjoints and $6 a^{2}+16\left(1-g_{a}\right)$ fundamental matter representations charged under the first $\mathrm{SU}(2)$, and similarly for the second $\mathrm{SU}(2)$ with $a \rightarrow b$, see (B.9). The number of bifundamental hypermultiplets will be $a b$. When both $\mathrm{SU}(2)$ groups are broken by Higgsing an adjoint representation, the remaining adjoints become charge 2 fields, and fundamentals become charge 1 fields under one of the two $\mathrm{U}(1)$ factors (and neutral under the other $\mathrm{U}(1)$ ) in the resulting $\mathrm{U}(1) \times \mathrm{U}(1)$ gauge theory. In the simplest case, $a=b=3$, there are 9 bifundamental fields that carry charges $( \pm 1, \pm 1)$ after Higgsing, and another 36 fundamental fields for each factor that carry charges $( \pm 1,0)$ and $(0, \pm 1)$ each. For each $\mathrm{U}(1)$ the total number of matter fields of charge \pm 1 is 108 , satisfying the anomaly cancellation conditions. Note that the counting of matter multiplets here is in terms of full $6 \mathrm{D}$ hypermultiplets, each of which contains two "half hypermultiplets" with opposite charges.

Note that this distribution of charges cannot be realized using the class of $U(1) \times U(1)$ models constructed in [8-12]. Thus, a more general model is needed.

\subsubsection{Higgsing $\mathrm{SU}(3)$}

In a similar vein, we can tune an $\mathrm{SU}(3)$ gauge group on a single smooth divisor $A \geq$ $-K_{B}$. The resulting $\mathrm{SU}(3)$ theory will have at least one adjoint matter representation, 


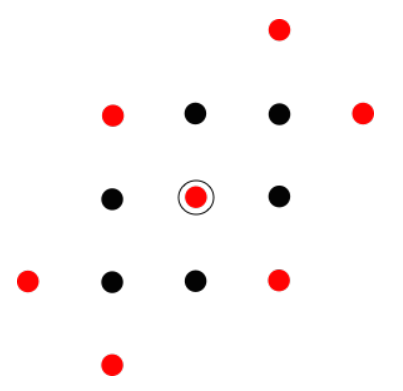

Figure 2. Weight lattice of charges in a theory with a $\mathrm{U}(1) \times \mathrm{U}(1)$ gauge group realized by Higgsing a theory with gauge group $\mathrm{SU}(3)$. Dots in red indicate the adjoint and black dots are the fundamental and antifundamental. The origin is indicated by an extra circle.

and some number of fundamental matter representations. Note that in the six-dimensional theory, with vector-like matter, the "fundamental" matter hypermultiplets contain both the fundamental and conjugate anti-fundamental representations. On $\mathbb{P}^{2}$, for example, we again have $g=(a-1)(a-2) / 2$ adjoint representations when the $\mathrm{SU}(3)$ is tuned on a degree $a$ curve, and the corresponding number of fundamental matter fields is $x_{3}=$ $6 a^{2}+18(1-g)$, cf. the general multiplicity formula (B.18). The $\mathrm{SU}(3)$ can be broken by Higgsing along two Cartan generators, e.g. $\lambda=\operatorname{diag}(1,-1,0), \mu=\operatorname{diag}(1,0,-1)$. The fundamental representation of $\mathrm{SU}(3)$ carries charges $(1,1),(-1,0)$, and $(0,-1)$ under the resulting $\mathrm{U}(1) \times \mathrm{U}(1)$, and the anti-fundamental representation in the same multiplet carries charges $(-1,-1),(1,0)$, and $(0,1)$. Additional adjoint representations of SU(3) acquire charges of $\pm(2,1), \pm(1,2)$, and $\pm(1,-1)$ (and two singlets) under the breaking to $\mathrm{U}(1) \times \mathrm{U}(1)$. This spectrum is shown graphically in figure 2 .

Again, this type of spectrum cannot be produced by the $\mathrm{U}(1) \times \mathrm{U}(1)$ models in [8-12].

As a simple example, consider tuning an $\mathrm{SU}(3)$ on a cubic on $\mathbb{P}^{2}$. The matter content consists of one adjoint and 54 fundamental matter fields. Breaking the $\mathrm{SU}(3)$ to $\mathrm{U}(1) \times \mathrm{U}(1)$ by Higgsing the adjoint gives 54 matter fields in each of the representations $(1,1),(1,0)$, $(0,1)$, which appear along with their complex conjugates $(-1,-1),(-1,0),(0,-1)$ in each full hypermultiplet. Note that again we have 108 charged matter fields under each U(1) factor, so anomaly cancellation is satisfied. There is, however, with this spectrum no way to redefine the basis of $U(1)$ fields to match the spectrum found in the class of theories realized by Higgsing an $\mathrm{SU}(2) \times \mathrm{SU}(2)$ model.

It is worth mentioning also here the spectrum that results if an $\mathrm{SU}(3)$ is partially Higgsed on a single Cartan generator to give a theory with $\mathrm{U}(1) \times \mathrm{SU}(2)$ gauge group. In this case, Higgsing for example on $\lambda_{8}=\operatorname{diag}(1,1,-2)$, the fundamental representation of $\mathrm{SU}(3)$ breaks to the representation content $(+1, \mathbf{2})+(-2, \mathbf{1})$ of $\mathrm{U}(1) \times \mathrm{SU}(2)$, with the anti-fundamental in the same hypermultiplet carrying the conjugate to this representation content, and the adjoint breaks to $(+3, \mathbf{2})+(0, \mathbf{3}),(-3, \mathbf{2})$. Note that all fields are invariant under the diagonal $\mathbb{Z}_{2}$, so that the precise gauge group in this case is $(\mathrm{U}(1) \times \mathrm{SU}(2)) / \mathbb{Z}_{2}$. The presence of this discrete $\mathbb{Z}_{2}$ quotient and the difference in spectrum distinguishes this representation content from that associated with the Higgsing of a single $\mathrm{SU}(2)$ in an $\mathrm{SU}(2) \times \mathrm{SU}(2)$ model such as discussed in the previous subsection. Breaking down to 
$\mathrm{U}(1) \times \mathrm{U}(1)$, it is straightforward to check that the representation content reproduces that described above, under an appropriate linear recombination of the generators.

\subsubsection{Hybrid models from $\mathrm{SU}(2) \times \mathrm{SU}(2) \times \mathrm{SU}(3)$}

While the preceding models are the simplest ways of getting two U(1) factors from the Higgsing of a non-Abelian theory, by considering the possibility of reducible divisors supporting the $\mathrm{SU}(2)$ factors as in the single $\mathrm{U}(1)$ theories described above, some more interesting structures can emerge. While for a single $\mathrm{U}(1)$ factor, the possible spectra are essentially the same whether the $\mathrm{U}(1)$ comes from Higgsing an $\mathrm{SU}(2)$ on an irreducible divisor $A$ or a reducible divisor $X=A B$, when there are two $\mathrm{U}(1)$ factors, we can consider a situation where one $\mathrm{U}(1)$ comes from Higgsing a non-Abelian factor on $X=A C$, and the other $\mathrm{U}(1)$ comes from Higgsing a non-Abelian factor on $Y=B C$. Compared to models with a single $\mathrm{U}(1)$ factor, much more interesting and subtle structure is possible when the reduction occurs in this way for the $\mathrm{U}(1) \times \mathrm{U}(1)$ model. In particular, in this situation the divisor $C$ generally will support a gauge factor $\mathrm{SU}(3)$, and we can get a variety of different charges in the $\mathrm{U}(1) \times \mathrm{U}(1)$ theory by starting with different matter content in the unHiggsed theory.

In fact, the general Abelian $\mathrm{U}(1)^{2}$ theory considered in this work is precisely of this type. It arises from Higgsing a non-Abelian theory with gauge group

$$
G=\mathrm{SU}(2) \times \mathrm{SU}(2) \times \mathrm{SU}(3),
$$

where the three factors are tuned on divisors $A, B, C$. Most notably, the gauge group on $C$ is only an $\mathrm{SU}(3)$ associated to an $I_{3}$ singularity, in contrast to the common expectation of an $I_{4}$ singularity at the collision of two $I_{2}$ singularities. In general, each gauge group factor will have some associated number of fundamental representations and some number of adjoint representations, and there can be bifundamental representations between each pair of gauge group factors. The multiplicities of these matter fields are given by the general formulae (B.22). In this general context, the bifundamental fields are primarily used to Higgs the product group $\mathrm{SU}(2) \times \mathrm{SU}(2) \times \mathrm{SU}(3)$ (which requires at least two bifundamentals for D-flatness), the spectrum of the resulting $\mathrm{U}(1) \times \mathrm{U}(1)$ model will be somewhat different from either of the rank two Higgsings described above.

As in the case of unHiggsing a single $\mathrm{U}(1)$ factor, any of the divisors $A, B, C$ can be reducible, leading to a larger gauge group after unHiggsing. We find one broad class of models where $A$ is generically reducible, so that the complete gauge group after unHiggsing is $\mathrm{SU}(2) \times \mathrm{SU}(2) \times \mathrm{SU}(2) \times \mathrm{SU}(3)$, and the Higgsing is again carried out through bifundamental fields.

To see how the matter spectrum of the theory follows from Higgsing the general SU(2) $\times \mathrm{SU}(2) \times \mathrm{SU}(3)$ model on bifundamental fields, consider first Higgsing on a bifundamental $(\mathbf{1}, \mathbf{2}, \mathbf{3})$ between the $\mathrm{SU}(2) \times \mathrm{SU}(3)$ on $B \cap C$, via a vacuum expectation value proportional to

$$
\left(\begin{array}{lll}
1 & 0 & 0 \\
0 & 0 & 0
\end{array}\right)
$$

This leaves a gauge group $\mathrm{SU}(2) \times \mathrm{U}(1) \times \mathrm{SU}(2)$. We can then Higgs a bifundamental $(\mathbf{2}, \mathbf{1}, \mathbf{2})$ on $A \cap C$, leaving a gauge group $\mathrm{U}(1) \times \mathrm{U}(1)$. Carrying this out explicitly, we can 
Figure 3. Weight lattice of charges in a theory with a $\mathrm{U}(1) \times \mathrm{U}(1)$ gauge group realized by Higgsing a theory with gauge group $\mathrm{SU}(2) \times \mathrm{SU}(2) \times \mathrm{SU}(3)$. The origin is indicated by an extra circle.

write the residual $\mathrm{U}(1)$ generators $\xi$ and $\zeta$ in terms of the Cartan generators of the factors $\mathrm{SU}(2), \mathrm{SU}(2), \mathrm{SU}(3)$ as

$$
\xi=\mu-\lambda-\sigma_{3}^{(1)}, \quad \zeta=\sigma_{3}^{(2)}-\lambda .
$$

A short calculation then shows that the spectra of the different bifundamentals decompose under $\mathrm{U}(1) \times \mathrm{U}(1)$ as

$$
\begin{aligned}
& (\mathbf{2}, \mathbf{2}, \mathbf{1}) \rightarrow( \pm 1, \pm 1) \\
& (\mathbf{1}, \mathbf{2}, \mathbf{3}) \rightarrow(-1,1)+(-1,-1)+(0,-2)+(1,0)+(1,2)+(0,0) \\
& (\mathbf{2}, \mathbf{1}, \mathbf{3}) \rightarrow(-1,-1)+(1,-1)+(-2,0)+(0,1)+(2,1)+(0,0) .
\end{aligned}
$$

Similarly, we obtain the following decomposition under $\mathrm{U}(1) \times \mathrm{U}(1)$ of the fundamentals

$$
\begin{aligned}
& (\mathbf{2}, \mathbf{1}, \mathbf{1}) \rightarrow( \pm 1,0) \\
& (\mathbf{1}, \mathbf{2}, \mathbf{1}) \rightarrow(0, \pm 1) \\
& (\mathbf{1}, \mathbf{1}, \mathbf{3}) \rightarrow(0,-1)+(1,1)+(1,0) .
\end{aligned}
$$

This spectrum is shown in figure 3, where we emphasize that a hypermultiplet in complex representation always contains states of a given charge and their conjugates. If there are adjoints on any of the divisors $A, B, C$ they will decompose as

$$
\begin{aligned}
& (\mathbf{3}, \mathbf{1}, \mathbf{1}) \rightarrow( \pm 2,0)+(0,0) \\
& (\mathbf{1}, \mathbf{3}, \mathbf{1}) \rightarrow(0, \pm 2)+(0,0) \\
& (\mathbf{1}, \mathbf{1}, \mathbf{8}) \rightarrow \pm(2,1)+ \pm(1,2)+ \pm(1,-1)+2(0,0)
\end{aligned}
$$

Thus, the Higgsing on bifundamentals of a theory with $\mathrm{SU}(2) \times \mathrm{SU}(2) \times \mathrm{SU}(3)$ gauge group gives a different, and more general, spectrum than the rank two Higgsings on $\mathrm{SU}(2) \times \mathrm{SU}(2)$ or $\mathrm{SU}(3)$ above.

\subsubsection{Singular divisors and higher charges}

In the general $\mathrm{U}(1) \times \mathrm{U}(1)$ Weierstrass model that we derive in the following section, it turns out that there are even more exotic classes of spectra, corresponding to Higgsing of 
models where a gauge group has been tuned on a singular divisor, associated with a more complicated matter representation.

While generic F-theory models in which gauge groups are tuned on smooth divisors give only simple matter representations for the low-energy gauge group, such as fundamental and antisymmetric representations for $\mathrm{SU}(N)$ gauge groups, more exotic representations can be realized in F-theory when the coefficients in the Weierstrass model are tuned to realize more complicated codimension two loci in the elliptic fibration. While there is not yet a complete map between codimension two singularities structures and the representation theory of matter and the corresponding low-energy supergravity theory, anomaly cancellation in $6 \mathrm{D}$ theories gives some insight into this correspondence, which has partially been explored in [41, 42]. One of the simplest examples of this kind of situation arises for representations of $\mathrm{SU}(N)$. As shown in [41], every irreducible representation $R$ of a simple gauge group factor $G$ has associated with it a genus contribution $g_{R}$. The value of $g_{R}$ should indicate the contribution to the arithmetic genus of a singular curve in the base surface of an F-theory compactification that carries that gauge group, where the matter representation $R$ is located at the singularity in question. All purely antisymmetric tensor representations of $\mathrm{SU}(N)$ (i.e., those whose Young diagram has a single column) have a genus contribution of $g=0$, and can be realized by codimension two singularities of the Weierstrass model over a smooth curve. The adjoint and symmetric tensor representations both carry genus contributions of $g=1$ for all $\mathrm{SU}(N)$. The adjoint representation is the matter representation carried generically by a smooth divisor of genus $g>0$. As described in $[42,43]$, the symmetric tensor representation arises when the gauge group is supported on a curve $C$ that has an ordinary double point or cusp singularity. For SU(2), the adjoint and symmetric tensor representations are equivalent, so this distinction does not lead to different spectra in low-energy theories either of an SU(2) or U(1) theory. For $\mathrm{SU}(3)$, on the other hand, the adjoint and symmetric tensor representations are distinct. In six-dimensional theories, anomaly cancellation conditions treat an adjoint matter representation as equivalent to the combination of a symmetric and an anti-symmetric tensor representation of $\mathrm{SU}(3)$. Thus, there are low energy theories of $6 \mathrm{D}$ supergravity with an $\mathrm{SU}(3)$ gauge group in which some $g^{\prime}$ of the total possible $g$ adjoint representations are replaced by symmetric + antisymmetric representations. When such an $\mathrm{SU}(3)$ is Higgsed, it gives rise to a distinctive pattern of charges for the resulting $\mathrm{U}(1) \times \mathrm{U}(1)$ theory. In the general model that we describe in the following section, we find that there is a parameter that determines the number of such singularities that must be present on the curve carrying an $\mathrm{SU}(3)$ gauge group. Here we summarize the consequences for the spectrum after Higgsing.

We consider the most general model, where the unHiggsed gauge group is $\mathrm{SU}(2) \times$ $\mathrm{SU}(2) \times \mathrm{SU}(3)$. We now assume, however, that the $\mathrm{SU}(3)$ carries in addition to $g-g^{\prime}$ adjoint fields, $g^{\prime}$ symmetric and $g^{\prime}$ antisymmetric representations. The antisymmetric representations are just the anti-fundamental, so give rise to the same $\mathrm{U}(1)$ charges as the fundamental representation described in the previous subsection. The symmetric representation 6 of SU(3), however, transforms differently and gives rise to charges

$$
(\mathbf{1}, \mathbf{1}, \mathbf{6}) \rightarrow(2,2)+(0,-2)+(-2,0)+(0,1)+(1,0)+(-1,-1)
$$




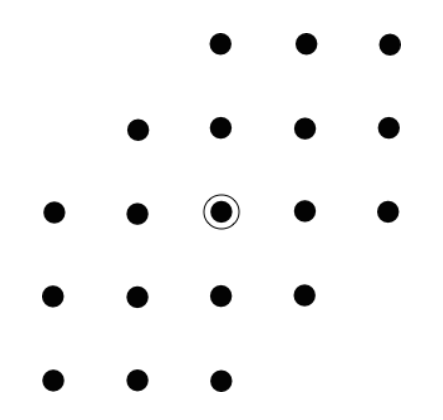

Figure 4. Weight lattice of charges in a theory with a $\mathrm{U}(1) \times \mathrm{U}(1)$ gauge group realized by Higgsing a theory with gauge group $\mathrm{SU}(2) \times \mathrm{SU}(2) \times \mathrm{SU}(3)$, when the $\mathrm{SU}(3)$ carries matter in the symmetric representation, associated in F-theory with singular points on the curve $C$ carrying the SU(3) factor.

Thus, in the most general situation we expect that we may have a spectrum of $\mathrm{U}(1) \times \mathrm{U}(1)$ charges such as those shown in figure 4.

\subsection{Examples}

To illustrate some of the different structures just described, we give some simple examples. These examples also show how the different branches of the set of $\mathrm{U}(1) \times \mathrm{U}(1)$ models have different dimensionalities.

We consider the simplest $6 \mathrm{D}$ models on the base $\mathbb{P}^{2}$ of each of the types just described.

For the $\mathrm{SU}(2) \times \mathrm{SU}(2)$ model, we have two $\mathrm{SU}(2)$ factors tuned on two cubic curves $A, B$. The spectrum of this model was described in section 2.2.1. After Higgsing both $\mathrm{SU}(2)$ factors as described there, using two adjoint fields, there are a total of $36+72+$ $72=180$ charged matter fields. Gravitational anomaly cancellation imposes the condition $H-V=273$, so the number of neutral scalar fields parameterizing this class of models is $H_{\text {uncharged }}=273+2-180=95$.

For the SU(3) model, we have a single SU(3) factor on a cubic curve $A$. We Higgs using the adjoint field to a $\mathrm{U}(1) \times \mathrm{U}(1)$ model. The spectrum as discussed above in section 2.2.2 now contains $54+54+54=162$ charged matter fields, so the number of neutral scalars is $H_{\text {uncharged }}=275-162=113$. Thus, this branch of the $\mathrm{U}(1) \times \mathrm{U}(1)$ moduli space has many more degrees of freedom than the branch arising from Higgsing the simplest $\mathrm{SU}(2) \times \mathrm{SU}(2)$ model.

Now we consider a hybrid model, where $\mathrm{SU}(2) \times \mathrm{SU}(2) \times \mathrm{SU}(3)$ is tuned on $A, B, C$, where $A, B$ are degree one (lines) and $C$ is degree two (conic). In this case in the nonAbelian theory there are no adjoint matter fields, and we Higgs the theory by turning on expectation values for bifundamentals as described in section 2.2.3. The numbers of charged and neutral hypermultiplets lie in-between those in the two models above: we have 172 charged fields and 103 neutral scalars. Because there are no adjoints of the $\mathrm{SU}(2)$ factors, and all the $\mathrm{SU}(2) \times \mathrm{SU}(3)$ bifundamentals are Higgsed, the set of charges is captured by the subset of charges that appear in both figure 1 and figure 2 ; if $A, B$ were higher degree the charge spectrum would be that of figure 3 . Note however that the detailed multiplicities in the spectrum resulting from this construction is different from any that could be produced simply by Higgsing an $\mathrm{SU}(3)$. In particular, in this model the 
spectrum contains 40 fields with charge $(1,1), 4$ fields with charge $(1,-1)$, and 64 fields each with charges $(1,0)$ and $(0,1)$ (in each case the hypermultiplet for the field also contains the conjugate charge). Here we used the branching rules (2.7)-(2.9) taking into account that two states each with charges $(-2,0),(0,-2),(1,2),(2,1)$ and $(-1,1)$ as well as two neutral singlets are eaten up, respectively. This resulting spectrum cannot be realized from the other constructions available.

Finally, we consider the simplest apparently consistent supergravity theory that would correspond to the spectrum depicted in figure 4, as described in section 2.2.4. In principle this could arise from an $\mathrm{SU}(3)$ with two adjoint matter representations, one symmetric representation, and 61 fundamental representations (of which one comes from the antisymmetric representation that comes along with the symmetric when replacing an adjoint). This model seems to give a sensible low-energy spectrum, and one might imagine that this spectrum could arise from an F-theory model where an $\mathrm{SU}(3)$ is tuned on a quartic curve $C$ with an appropriate double point singularity. Strangely, however, as we see later in this paper, the general construction we present here only gives models of this type associated with curves of degree 5 and higher. This puzzle is discussed further throughout the paper.

\section{General elliptic fibrations with three sections}

In this section we construct a general class of elliptically fibered Calabi-Yau manifolds $\pi: X_{n+1} \rightarrow B_{n}$ over a base $B_{n}$ so that the general elliptic fiber $\mathcal{E}=\pi^{-1}(p)$ with $p$ a point in $B_{n}$ has three rational points, i.e., there is a rank two Mordell-Weil group. F-theory compactified on $X_{n+1}$ then yields a low-energy effective theory with a $\mathrm{U}(1) \times \mathrm{U}(1)$ gauge symmetry. First, in section 3.1 we construct the elliptic curve $\mathcal{E}$ as a specialized cubic in $\mathbb{P}^{2}$. Then, we find its Weierstrass form as well as the Weierstrass coordinates of its rational points in section 3.2. Finally, in section 3.3, we construct all Calabi-Yau elliptic fibrations $X_{n+1}$ of $\mathcal{E}$ over $B_{n}$. The construction we describe here is completely generic and is valid for an arbitrary F-theory base manifold $B_{n}$.

The crucial difference between the construction here and the one in $[8-12,32]$ is that the rational points in $\mathcal{E}$ are kept at general positions and are not assumed to be toric points in $\mathbb{P}^{2}$ (i.e. the simultaneous vanishings of two homogeneous coordinates). This will be the key to constructing a more general model that can, for example, be unHiggsed to $\mathrm{SU}(2) \times \mathrm{SU}(2)$ and $\mathrm{SU}(3)$

\subsection{A new elliptic curve}

We consider an elliptic curve $\mathcal{E}$ with three rational points $P, Q$ and $R$. It is well-known due to Deligne [44], that these rational points define a line bundle $\mathcal{M}=\mathcal{O}(P+Q+R)$ that embeds $\mathcal{E}$ into $\mathbb{P}^{2}$ with $\mathcal{M}=\left.\mathcal{O}_{\mathbb{P}^{2}}(1)\right|_{\mathcal{E}}$, cf. [8-12]. In terms of the projective coordinates $[u: v: w]$ on $\mathbb{P}^{2}$, this embedding is given by the cubic

$$
p:=s_{1} u^{3}+s_{2} u^{2} v+s_{3} u v^{2}+s_{4} v^{3}+\left(s_{5} u^{2}+s_{6} u v+s_{7} v^{2}\right) w+\left(s_{8} u+s_{9} v\right) w^{2}+s_{10} w^{3}=0,
$$

where the coefficients $s_{i}$ take values in a given field $K$. In an elliptic fibration $\pi: X_{n+1} \rightarrow$ $B_{n}$ of $\mathcal{E}$, we identify $K$ with the field of rational functions on $B_{n}$. 


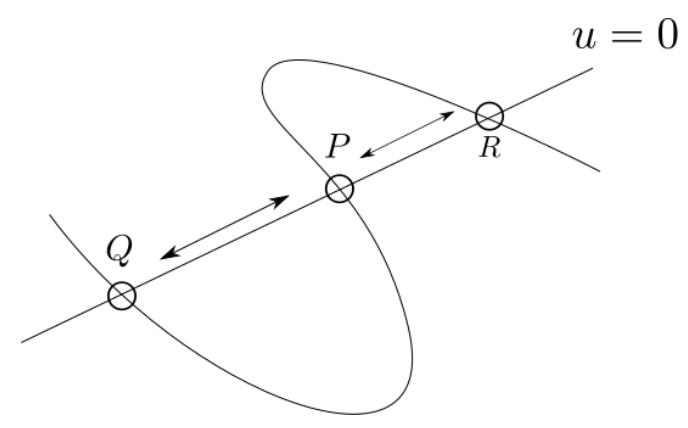

Figure 5. Cubic $\mathcal{E}$ with three rational points $P, Q$ and $R$ contained in the line $u=0$.

The identification $\mathcal{M}=\left.\mathcal{O}_{\mathbb{P}^{2}}(1)\right|_{\mathcal{E}}$ implies that there exists a section $U$ of $\mathcal{O}_{\mathbb{P}^{2}}(1)$ on the ambient space $\mathbb{P}^{2}$, so that $\left.U\right|_{\mathcal{E}}$ vanishes precisely at the three points $P, Q$ and $R .{ }^{5}$ By a rotation in the $\mathbb{P}^{2}$, we choose $U=u$, see figure $5 .{ }^{6}$ By setting $u=0$ in (3.1), we obtain a cubic in $v, w$,

$$
q_{3}=s_{4} v^{3}+s_{7} v^{2} w+s_{9} v w^{2}+s_{10} w^{3},
$$

which has to have three distinct roots in $K$ that are the rational points $P, Q$ and $R$. For elliptic fibrations over $B_{n}$, the field $K$ is in general not algebraically closed. Thus, the existence of these roots constrains the coefficients in $q_{3}$ by imposing it to factorize as

$$
q_{3}=\prod_{i=1}^{3}\left(a_{i} v+b_{i} w\right)
$$

where $a_{i}, b_{i}$ take values in $K$. Thus, the coefficients in $q_{3}$ have to be given by the elementary symmetric polynomials in the $a_{i}, b_{j}$. In summary, the elliptic fibration (3.1) meeting the requirement of admitting three rational points takes the form

$$
p=u\left(s_{1} u^{2}+s_{2} u v+s_{3} v^{2}+s_{5} u w+s_{6} v w+s_{8} w^{2}\right)+\prod_{i=1}^{3}\left(a_{i} v+b_{i} w\right)=0 .
$$

It is important to note that in an elliptic fibration it is not generally possible globally to move two of the roots of (3.3) to $v=0$ and $w=0$, as the necessary variable transformation will generically involve denominators that are ill-defined at certain loci on $B_{n}$. In contrast, for a single elliptic curve this is always possible and leads to the elliptic curve in $d P_{2}$ studied in an F-theory context in [8-12].

\footnotetext{
${ }^{5}$ Indeed the given points $P$ and $Q$ on $\mathcal{E}$ uniquely define a hyperplane in $\mathbb{P}^{2}$. This hyperplane intersects the cubic (3.1) in a third point, which automatically is rational. Thus, this point has to be $R$, because otherwise $\mathcal{E}$ would have a fourth rational point, which is excluded by construction.

${ }^{6}$ A general $U$ takes the form $U=A u+B v+C w$ with $A, B, C$ coefficients in appropriate line bundles on $B_{n}$. For a single elliptic curve, these are constants in $K$ and we can perform a Gl(3)-transformation so that $U=u$. In an elliptic fibration, this is also possible in a general class of models. For a fibration over a three-dimensional base $B_{3}$, one might worry about the codimension three locus $A=B=C$ in the base where $U \equiv 0$ which would be a point of $I_{3}$ fiber. However, we can use the $\mathbb{C}^{*}$-action on $\mathbb{P}^{2}$ to set e.g. $A \equiv 1$ globally. Since $u$ is then a coordinate on a $\mathbb{P}^{2}$-fiber independent of the base $B_{n}$, it follows that $U$ is non-vanishing on $B_{n}$, too. Thus, we can globally in the $\mathbb{P}^{2}$-fibration over $B_{n}$ perform the rotation to $U=u$.
} 
The three rational points $P, Q$ and $R$ of (3.4) have the general coordinates

$$
P=\left[0:-b_{1}: a_{1}\right], \quad Q=\left[0:-b_{2}: a_{2}\right], \quad R=\left[0:-b_{3}: a_{3}\right] .
$$

We note that these rational points coincide when the two lines $a_{i} v+b_{i} w=0$ and $a_{j} v+b_{j} w=$ 0 align. This happens when the $2 \times 2$-matrix of coefficients of these two equations has rank one, which is the case if $a_{i} b_{j}-a_{j} b_{i}=0$, for $i \neq j$. More explicitly, we obtain

$$
\begin{aligned}
& P=Q: a_{1} b_{2}-a_{2} b_{1}=0, \quad P=R: a_{1} b_{3}-a_{3} b_{1}=0, \quad Q=R: a_{2} b_{3}-a_{3} b_{2}=0 \\
& P=Q=R: a_{1} b_{2}-a_{2} b_{1}=a_{1} b_{3}-a_{3} b_{1}=a_{2} b_{3}-a_{3} b_{2}=0 .
\end{aligned}
$$

Note that we have to have $\left(a_{i}, b_{i}\right) \neq(0,0)$ because otherwise the elliptic curve (3.4) would not be smooth and the rational points not well-defined.

\subsubsection{Specialized models}

There are certain elliptic fibrations, for which the model (3.4) of the elliptic curve $\mathcal{E}$ can be further simplified. Indeed, if for a particular $i$ we have that $a_{i}$ is a constant (this is equivalent to the case of constant $b_{i}$ by exchanging $v$ and $w$ ), we can perform the global variable transformation

$$
v \mapsto v-\frac{b_{i}}{a_{i}} w,
$$

which effectively removes $b_{i}$ so that one root of $q_{3}$ in (3.3) is at $v=0$. Without loss of generality we can assume that $i=1$ and $a_{1}=1$ so that the hypersurface equation (3.4) takes the form

$$
u\left(s_{1} u^{2}+s_{2} u v+s_{3} v^{2}+s_{5} u w+s_{6} v w+s_{8} w^{2}\right)+v\left(a_{2} v+b_{2} w\right)\left(a_{3} v+b_{3} w\right)=0 .
$$

The coordinate (3.5) of the rational points reduce to

$$
P=[0: 0: 1], \quad Q=\left[0:-b_{2}: a_{2}\right], \quad R=\left[0:-b_{3}: a_{3}\right] .
$$

Note that in elliptic fibrations of this form the point $P$ is well defined everywhere on $B_{n}$. For this specialized model, we have also worked out the presentation as a non-generic quartic in $\mathbb{P}^{2}(1,1,2)$ in appendix $C$, which is a specialization of the $U(1)$ model of [4].

We emphasize two main differences between this specialized model and the general model (3.4). First of all, the coefficient $b_{1}$ is absent from the cubic (3.8). As we demonstrate in section 3.3, this implies that there is no corresponding effective divisor class associated to $b_{1}$, which gives us more freedom for the degrees of other divisor classes defining the elliptic fibration. Second, and more physically, F-theory compactifcations on elliptic fibrations based on (3.8) do not have matter fields at $a_{1}=b_{1}=0$, while the spectrum is otherwise identical, as we will work out in section 4.2.

Finally, we note that we can have a further specialization if in addition to a constant $a_{1}$, also $b_{2}$ is a constant. In this case, we can redefine $w$ globally to obtain the cubic

$$
u\left(s_{1} u^{2}+s_{2} u v+s_{3} v^{2}+s_{5} u w+s_{6} v w+s_{8} w^{2}\right)+v w\left(a_{3} v+b_{3} w\right)=0 .
$$


This is precisely the elliptic curve in $d P_{2}$ studied in [8-12]. $d P_{2}$-elliptic fibrations in general do not admit rank-preserving unHiggsings as the points at $u=v=0$ and $u=w=0$ generally do not collide in the fibration and, consequently, can not be merged by a tuning of the complex structure. As shown in [32], the theory can, however, be unHiggsed to $\mathrm{SU}(3)^{3} / \mathbb{Z}_{3}$ and $\left(\mathrm{SU}(2)^{2} \times \mathrm{SU}(4)\right) / \mathbb{Z}_{2}$, respectively, where the rational point $u=w=0$ has turned into Mordell-Weil torsion. ${ }^{7}$

In summary, we see that (3.4) admits certain specializations. As the case of constant $a_{1}$ and $b_{2}$ reduces to the elliptic curve in $d P_{2}$, that has been studied extensively in recent literature, we will primarily consider the completely general case and the novel special case $a_{1}=1, b_{1}=0$ with generic $a_{2}, a_{3}, b_{2}, b_{3}$.

\subsection{The Weierstrass form}

We now proceed with the calculation of the Weierstrass form (WSF)

$$
y^{2}=x^{3}+f x z^{4}+g z^{6}
$$

of the elliptic curve (3.4). Choosing $P$ as the zero point, the birational map to the WSF can be obtained by applying again the procedure of Deligne and constructing the sections of the line bundles $\mathcal{O}(n P)$ for $n=1, \ldots, 6$ yielding an embedding of $\mathcal{E}$ into $\mathbb{P}^{1,2,3}$. This procedure has been described and applied in an F-theory context recently, see appendix B of [4] and more specifically $[9,11]$ for the case of cubics. Alternatively, we can apply Nagell's algorithm to (3.4), see e.g. appendix B of [9].

Instead of following these two procedures, we take a shortcut here by using the WSF of the elliptic curve in $d P_{2}$. Indeed, we can perform the rational variable transformation $v \mapsto v-\frac{b_{1}}{a_{1}} w$ and $w \mapsto w-\frac{a_{1} a_{2}}{a_{1} b_{2}-a_{2} b_{1}} v$ on the cubic equation (3.4) so that it assumes the standard form of an $d P_{2}$-elliptic fibration, that is

$$
p_{\mathrm{dP}_{2}}=u\left(\tilde{s}_{1} u^{2}+\tilde{s}_{2} u v+\tilde{s}_{3} v^{2}+\tilde{s}_{5} u w+\tilde{s}_{6} v w+\tilde{s}_{8} w^{2}\right)+v w\left(\tilde{s}_{7} v+\tilde{s}_{9} w\right) .
$$

Here the coefficients $\tilde{s}_{i}$ are given by $\tilde{s}_{1}=s_{1}$ and

$$
\begin{array}{ll}
\tilde{s}_{2}=\frac{a_{1}\left(b_{2} s_{2}-a_{2} s_{5}\right)}{a_{1} b_{2}-a_{2} b_{1}}, & \tilde{s}_{3}=\frac{a_{1}^{2}\left(a_{2}^{2} s_{8}-a_{2} b_{2} s_{6}+b_{2}^{2} s_{3}\right)}{\left(a_{1} b_{2}-a_{2} b_{1}\right)^{2}}, \\
\tilde{s}_{5}=s_{5}-\frac{b_{1} s_{2}}{a_{1}}, & \tilde{s}_{6}=\frac{a_{1}\left(b_{2} s_{6}-2 a_{2} s_{8}\right)+b_{1}\left(a_{2} s_{6}-2 b_{2} s_{3}\right)}{a_{1} b_{2}-a_{2} b_{1}}, \\
\tilde{s}_{7}=a_{1}\left(a_{3} b_{2}-a_{2} b_{3}\right), & \tilde{s}_{8}=\frac{b_{1}\left(b_{1} s_{3}-a_{1} s_{6}\right)}{a_{1}^{2}}+s_{8} \\
\tilde{s}_{9}=\frac{\left(a_{1} b_{2}-a_{2} b_{1}\right)\left(a_{1} b_{3}-a_{3} b_{1}\right)}{a_{1}} &
\end{array}
$$

Then, we can directly apply the results of [9] for the functions $f, g$ of the Weierstrass model. Clearing denominators gives a Weierstrass model where the coefficients $f, g$ are polynomials in the $s_{i}, a_{j}, b_{k}$. The resulting expressions are quite lengthy and can be found in appendix A. We emphasize that both $f$ and $g$ are both manifestly symmetric under

\footnotetext{
${ }^{7}$ The subset of $d P_{2}$ models unHiggsing to $\mathrm{SU}(3) \times \mathrm{SU}(2)$ are discussed in section 4.3.
} 
exchange of the rational points, i.e. both expressions (A.1) and (A.2) are invariant under exchanging $\left(a_{i}, b_{i}\right) \leftrightarrow\left(a_{j}, b_{j}\right)$ for any pair $i \neq j$. Note that neither $f, g$ nor the discriminant $\Delta$ admit a factorization, signaling the absence of codimension one singularities. The singularities at codimension two and higher will be discussed in section 4.1.

We can use this birational map to WSF in order to compute the Weierstrass coordinates of the rational points $Q, R$. Again, the obtained formula are lengthy and relegated to appendix A. For $Q$ we obtain the coordinates given in (A.3). The Weierstrass coordinates of the rational point $R$, given in (A.4) and denoted by $\left[y_{R}: x_{R}: z_{R}\right]$, are obtained from the ones for $Q$ by exchanging the variables $\left(a_{2}, b_{2}\right) \leftrightarrow\left(a_{3}, b_{3}\right)$ by symmetry of the elliptic curve (3.4).

Specialized model. The WSF of the specialized cubic curve (3.8) is obtained from (A.1), (A.2) by setting $a_{1}=1$ and $b_{1}=0$. Similarly we obtain the Weierstrass coordinates of the rational point $Q$ from (A.3) in this limit:

$$
\begin{aligned}
z_{Q}= & -b_{2}, \\
x_{Q}= & \frac{1}{12}\left(-4 b_{2}^{3}\left(b_{3} s_{2}+a_{3} s_{5}\right)-12 a_{2} b_{2} s_{6} s_{8}+12 a_{2}^{2} s_{8}^{2}+b_{2}^{2}\left(8 a_{2} b_{3} s_{5}+s_{6}^{2}+8 s_{3} s_{8}\right)\right), \\
y_{Q}= & \frac{1}{2}\left(b_{2}^{4} b_{3}\left(a_{3} b_{2}-a_{2} b_{3}\right) s_{1}-b_{2}^{2}\left(a_{3} b_{2}-a_{2} b_{3}\right)\left(b_{2} s_{2}-a_{2} s_{5}\right) s_{8}-b_{2}^{2} b_{3} s_{5}\left(b_{2}^{2} s_{3}-a_{2} b_{2} s_{6}+a_{2}^{2} s_{8}\right)\right. \\
& \left.\quad+s_{8}\left(b_{2} s_{6}-2 a_{2} s_{8}\right)\left(b_{2}^{2} s_{3}-a_{2} b_{2} s_{6}+a_{2}^{2} s_{8}\right)\right) .
\end{aligned}
$$

As before, we obtain the coordinates of the point $R$, by symmetry of (3.8), upon exchanging $\left(a_{2}, b_{2}\right) \leftrightarrow\left(a_{3}, b_{3}\right)$.

\subsection{Constructing elliptic fibrations}

We proceed with the construction of elliptically fibered Calabi-Yau manifolds $\pi: X_{n+1} \rightarrow$ $B_{n}$ as fibrations of the curve $\mathcal{E}$ defined in (3.4) over an arbitrary base $B_{n}$. Although we focus in this work on the case of Calabi-Yau threefolds, the discussion in this section applies to any complex dimension of $X_{n+1}$. The procedure outlined here has been used already, e.g., in $[9,13,32]$, to which we refer the reader for more details.

An elliptic fibration by the curve $\mathcal{E}$ over a base $B_{n}$ is formally obtained by identifying the field $K$ with the field of rational functions on $B_{n}$. Upon cancelling denominators, the coefficients $s_{i}, a_{j}$ and $b_{j}$ in (3.4) are identified with sections of appropriate line bundles over $B_{n}$. The correct line bundles are determined by imposing the Calabi-Yau condition on the total space of the fibration. Clearly, in the obtained elliptic fibration $X_{n+1}$, the rational points in (3.5) lift to rational sections of the fibration, that we denote by $\hat{s}_{P}$, $\hat{s}_{Q}$ and $\hat{s}_{R}$. Their homology classes give rise to two $\mathrm{U}(1)$ gauge symmetries in F-theory compactifications on $X_{n+1}[3]$.

In more detail, we first fiber the two dimensional ambient space $\mathbb{P}^{2}$ of (3.4) over $B_{n}$, yielding the projective bundle

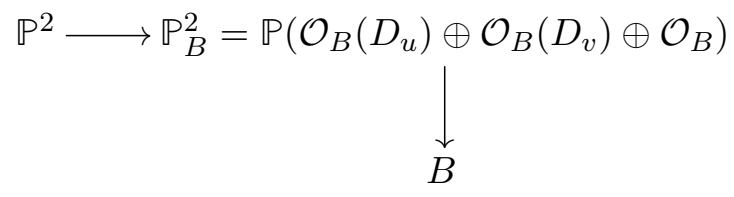


This is fully specified by the two divisors $D_{u}$ and $D_{v}$ on $B_{n}$, respectively, their associated line bundles being $\mathcal{O}_{B}\left(D_{u}\right)$ and $\mathcal{O}_{B}\left(D_{v}\right)$, which we chose so that

$$
u \in \mathcal{O}\left(D_{u}\right), \quad v \in \mathcal{O}\left(D_{v}\right)
$$

This implies that the constraint (3.4) becomes a section of a line bundle over the base $B_{n}$. Consistency, i.e. the requirement that every monomial in (3.4) transforms as a section of the same line bundle, then requires that the coefficients $s_{i}, a_{j}$ and $b_{j}$ are sections of appropriate line bundles. Finally, we require in addition that the constraint (3.4) is a section of the anti-canonical bundle of (3.15), i.e., defines a Calabi-Yau manifold $X_{n+1}$. This condition fixes the line bundles for the coefficients $s_{i}, a_{j}$ and $b_{j}$.

Computing the anti-canonical bundle of (3.15) by adjunction and solving the constraints imposed by the Calabi-Yau condition, we obtain that the coefficients $s_{i}, a_{j}, b_{j}$ have to be sections in the line bundles

\begin{tabular}{|c|c|}
\hline Section & Line bundle \\
\hline$s_{1}$ & $\mathcal{O}\left(-6 K_{B}-2\left[a_{1}\right]-2\left[a_{2}\right]-2\left[a_{3}\right]-3\left[s_{8}\right]\right)$ \\
$s_{2}$ & $\mathcal{O}\left(-4 K_{B}-\left[a_{1}\right]-\left[a_{2}\right]-\left[a_{3}\right]-2\left[s_{8}\right]\right)$ \\
$s_{3}$ & $\mathcal{O}\left(-2 K_{B}-\left[s_{8}\right]\right)$ \\
$s_{5}$ & $\mathcal{O}\left(-3 K_{B}-\left[a_{1}\right]-\left[a_{2}\right]-\left[a_{3}\right]-\left[s_{8}\right]\right)$ \\
$s_{6}$ & $\mathcal{O}\left(-K_{B}\right)$ \\
$s_{8}$ & $\mathcal{O}\left(\left[s_{8}\right]\right)$ \\
$a_{1}$ & $\mathcal{O}\left(\left[a_{1}\right]\right)$ \\
$a_{2}$ & $\mathcal{O}\left(\left[a_{2}\right]\right)$ \\
$a_{3}$ & $\mathcal{O}\left(\left[a_{3}\right]\right)$ \\
$b_{1}$ & $\mathcal{O}\left(K_{B}+\left[a_{1}\right]+\left[s_{8}\right]\right)$ \\
$b_{2}$ & $\mathcal{O}\left(K_{B}+\left[a_{2}\right]+\left[s_{8}\right]\right)$ \\
$b_{3}$ & $\mathcal{O}\left(K_{B}+\left[a_{3}\right]+\left[s_{8}\right]\right)$ \\
\hline
\end{tabular}

Here $-K_{B}$ is the anti-canonical divisor of $B_{n}$, and we set $D_{u}=3 K_{B}+\left[a_{1}\right]+\left[a_{2}\right]+\left[a_{3}\right]+2\left[s_{8}\right]$, $D_{v}=K_{B}+\left[s_{8}\right]$ so that all base divisors are parametrized by the classes $\left[a_{i}\right], i=1,2,3$, and $\left[s_{8}\right]$, corresponding to the sections $a_{i}$ and $s_{8}$, respectively. This parametrization is convenient as these four classes have to be effective in a generic model. They can chosen freely subject to the condition that all $s_{i}$ and $a_{j}, b_{j}$ are effective, which yields a finite discrete set of possible strata in the moduli space of $X_{n+1}$. We note that the line bundles for each of the parameters in (3.17) can also be determined efficiently directly from the form of the Weierstrass coefficients $f, g$ in appendix A, where $f \in \mathcal{O}\left(-4 K_{B}\right), g \in \mathcal{O}\left(-6 K_{B}\right)$.

As immediate consequences of the effectiveness condition of (3.17) we infer that

$$
\left[s_{8}\right] \leq-2 K_{B}, \quad-K_{B} \leq\left[a_{i}\right]+\left[s_{8}\right], \quad\left[a_{1}\right]+\left[a_{2}\right]+\left[a_{3}\right]+\left[s_{8}\right] \leq-3 K_{B},
$$

where $-K_{B}$ must be $(\mathbb{Q}-)$ effective for a Calabi-Yau Weierstrass model to exist. These conditions will be crucial for the understanding of the unHiggsings of the $\mathrm{U}(1)$-symmetries by tuning of the parameters in (3.4) as discussed below. 
In summary, we see that the elliptically fibered Calabi-Yau manifold $X_{n+1}$ is specified by four discrete parameters. We note that this additional freedom is not present in the earlier construction in [8-12], where there are only two discrete parameters. The presence of two additional discrete parameters is expected for the cubic in (3.2): it correspond to the two discrete degrees of freedom reflected in the two independent pairwise rescalings $\left(\left(a_{i}, b_{i}\right),\left(a_{j}, b_{j}\right)\right) \mapsto\left(\lambda\left(a_{i}, b_{i}\right), \lambda^{-1}\left(a_{j}, b_{j}\right)\right)(i \neq j)$ which leave $p=0$ invariant, where $\lambda$ is a section of a line-bundle on $B_{n}$. The additional discrete choices arise from specifying these two line-bundles.

The specialized model. The case of the specialized model (3.8) is covered by the general result (3.17) by setting $\left[a_{1}\right]=0$ and dropping the coefficient $b_{1}$, as this is no longer present in the model. We obtain

\begin{tabular}{|c|c|}
\hline Section & Line bundle \\
\hline$s_{1}$ & $\mathcal{O}\left(-6 K_{B}-2\left[a_{2}\right]-2\left[a_{3}\right]-3\left[s_{8}\right]\right)$ \\
$s_{2}$ & $\mathcal{O}\left(-4 K_{B}-\left[a_{2}\right]-\left[a_{3}\right]-2\left[s_{8}\right]\right)$ \\
$s_{3}$ & $\mathcal{O}\left(-2 K_{B}-\left[s_{8}\right]\right)$ \\
$s_{5}$ & $\mathcal{O}\left(-3 K_{B}-\left[a_{2}\right]-\left[a_{3}\right]-\left[s_{8}\right]\right)$ \\
$s_{6}$ & $\mathcal{O}\left(-K_{B}\right)$ \\
$s_{8}$ & $\mathcal{O}\left(\left[s_{8}\right]\right)$ \\
$a_{2}$ & $\mathcal{O}\left(\left[a_{2}\right]\right)$ \\
$a_{3}$ & $\mathcal{O}\left(\left[a_{3}\right]\right)$ \\
$b_{2}$ & $\mathcal{O}\left(K_{B}+\left[a_{2}\right]+\left[s_{8}\right]\right)$ \\
$b_{3}$ & $\mathcal{O}\left(K_{B}+\left[a_{3}\right]+\left[s_{8}\right]\right)$ \\
\hline
\end{tabular}

We emphasize that the absence of the coefficient $b_{1}$ weakens the effectiveness conditions imposed by (3.19) on the remaining parameters $\left[a_{2}\right],\left[a_{3}\right]$ and $\left[s_{8}\right]$, compared to (3.17). In fact, we do not have the constraint $-K_{B} \leq\left[s_{8}\right]$. This implies that the model (3.8) has qualitatively different unHiggsings than the general model (3.4).

\subsection{Comparison with $d P_{2}$-elliptic fibrations}

Before continuing with our discussion, let us pause to compare with the elliptic fibrations with rank two Mordell-Weil group constructed in [8-12] using the elliptic curve in $d P_{2}$.

Clearly, for a single elliptic curve over the complex numbers $\mathbb{C}$, we can always redefine the coordinates $[u: v: w]$ in (3.4) to map to the elliptic curve in $d P_{2}$ given in (3.10). However, in an elliptic fibration, the necessary coordinate redefinition is not defined globally unless we specialize $\left[a_{1}\right]=\left[b_{2}\right]=0$. Then, we can perform a coordinate redefinition that effectively sets $b_{1}=a_{2}=0$. Like the specialized model described in (3.19), the $d P_{2}$-elliptic fibration gives a class of constructions that are not captured by the general model $X_{n+1}$ where all coefficients $a_{i}, b_{i}$ are non-vanishing. In the $d P_{2}$-elliptic fibration, the coefficients $b_{1}$ and $a_{2}$ are absent. Consequently, their divisor classes in (3.17) do not have to be effective, which relaxes the constraints imposed on the remaining two parameters $\left[a_{3}\right]$ and $\left[s_{8}\right]$, which are now allowed to assume values that would violate the effectiveness of $b_{1}$ and $a_{2}$ if those 

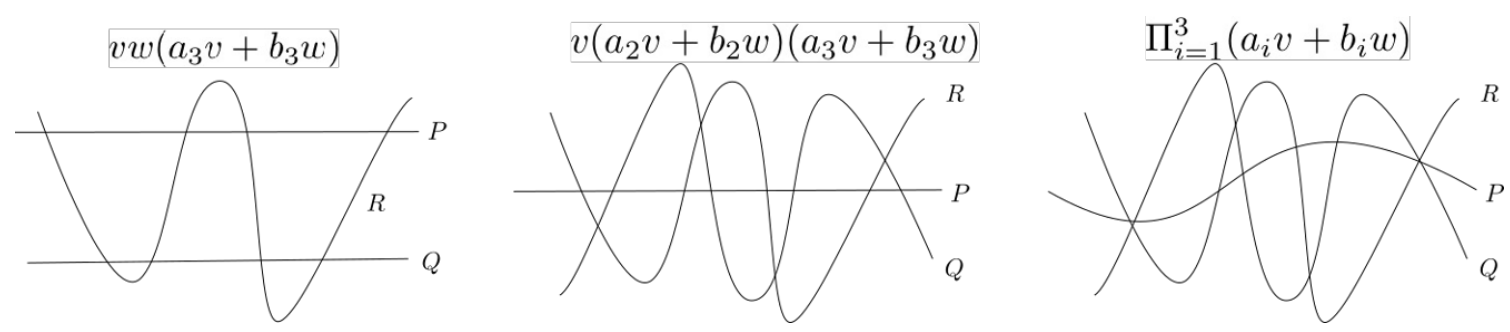

Figure 6. Intersection pattern of sections in three different cubic fibers.

parameters were non-vanishing. Hence, we obtain new models based on the $d P_{2}$-elliptic curve giving rise to different physics in F-theory, that are not just a specialization of $\mathrm{F}$ theory on a general $X_{n+1}$. Note, however, that all the models found in the specialized model (3.19) and the $d P_{2}$ construction are still special cases of the Weierstrass model described in appendix A; the distinguishing feature of these specialized models is that a broader range of classes can be chosen for the non-vanishing parameters $\left[a_{3}\right],\left[s_{8}\right]$, and $\left[a_{2}\right]$ in the specialized models.

Another way to formulate the difference between elliptic fibrations based on (3.4) with generic coefficients and those based on the specialized model (3.8) or the $d P_{2}$-curve (3.10) is to compare the general intersection patterns of the rational sections in these three models. The intersection pattern of the sections in elliptic fibrations based on $d P_{2}$ is shown in the first figure in figure 6 , that of the specialized models in the second figure while a generic situation in the general model $X_{n+1}$ is shown in the third figure. It is observed that the sections $\hat{s}_{P}$ and $\hat{s}_{Q}$ in $d P_{2}$-models can not intersect at codimension one in the base $B_{n}$ as they are induced by the fixed toric points $P=[0: 0: 1]$ and $Q=[0: 1: 0]$. In contrast, in a specialized model, all sections can intersect independently, as discussed in (3.6). Finally, in a general model all sections can intersect, too, and the zero section is no longer rigid. As we will demonstrate in section 4.2, this gives rise to a different matter spectrum of the F-theory models constructed as $d P_{2}$-elliptic fibrations, the specialized and the general Calabi-Yau manifolds $X_{n+1}$; in particular, the disjoint nature of the sections $P, Q$ in the $d P_{2}$-models indicates that these sections cannot be brought together to form an unHiggsed model without Abelian factors in a straightforward fashion.

\section{Singularities, global resolution and the matter spectrum}

In this section, we discuss the singularities of the elliptic fibration of the Calabi-Yau manifold $X_{n+1}$, the construction of a global CY-resolution of these singularities and the determination of the matter spectrum of the corresponding F-theory model. As we discuss further in section 4.1, the relevant singularities of $X_{n+1}$ are at least codimension two in the base $B_{n}$. The global resolution is represented as a complete intersection in a $(n+4)$-dimensional ambient space. It describes two blow-ups at two non-toric points in the ambient space of the elliptic fiber. In section 4.2, we use these results to obtain the general matter spectrum of an 6D F-theory compactification on $X_{n+1}$. 


$I_{2}$-loci at codimension two in $B_{n}$
$V\left(I_{(1)}\right):=\left\{a_{1}=b_{1}=0\right\}$
$V\left(I_{(2)}\right):=\left\{a_{2}=b_{2}=0\right\}$
$V\left(I_{(3)}\right):=\left\{a_{3}=b_{3}=0\right\}$
$V\left(I_{(4)}\right):=\left\{\Delta_{12}=s_{3} b_{1}^{2}-s_{6} a_{1} b_{1}+s_{8} a_{1}^{2}=0\right\} \backslash V\left(I_{(1)}\right)$
$V\left(I_{(5)}\right):=\left\{\Delta_{13}=s_{3} b_{1}^{2}-s_{6} a_{1} b_{1}+s_{8} a_{1}^{2}=0\right\} \backslash V\left(I_{(1)}\right)$
$V\left(I_{(6)}\right):=\left\{\Delta_{23}=s_{3} b_{2}^{2}-s_{6} a_{2} b_{2}+s_{8} a_{2}^{2}=0\right\} \backslash V\left(I_{(2)}\right)$
$V\left(I_{(7)}\right):=\left\{y_{Q}=y_{R}=\left(z_{Q}\right)^{4} f+3\left(x_{Q}\right)^{2}=\left(z_{R}\right)^{4} f+3\left(x_{R}\right)^{2}=0\right\} \backslash V\left(I_{(1)}\right)$
$V\left(I_{(8)}\right):=\left\{y_{Q}=\left(z_{Q}\right)^{4} f+3\left(x_{Q}\right)^{2}=0\right\} \backslash V\left(I_{(1)} \cdot I_{(2)} \cdot I_{(4)} \cdot I_{(5)} \cdot I_{(6)} \cdot I_{(7)}\right)$
$V\left(I_{(9)}\right):=\left\{y_{R}=\left(z_{R}\right)^{4} f+3\left(x_{R}\right)^{2}=0\right\} \backslash V\left(I_{(1)} \cdot I_{(3)} \cdot I_{(4)} \cdot I_{(5)} \cdot I_{(6)} \cdot I_{(7)}\right)$

Table 1. Codimension two matter loci of $X_{n+1}$.

Note that in the discussion here we focus on the matter charged under the U(1) Ftheory fields associated with the rank two Mordell-Weil group, i.e. matter whose existence is imposed by the considered elliptic fiber of the form (3.4). For simplicity, in this analysis we may assume that we are considering a generic elliptic fibration over a weak Fano base, so that there are no non-Abelian gauge group factors. As described in [45-47] for 6D and $4 \mathrm{D}$ F-theory compactifications, most bases $B_{n}$ are not weak Fano, and have associated nonHiggsable non-Abelian gauge group factors for generic elliptic fibrations. In such cases, or when the Weierstrass model over a Fano base is specifically tuned, there are codimension one singularities as well, and a full resolution would involve non-Abelian gauge groups and associated charged matter as well as the Abelian factors and Abelian charges we describe here. More detailed analysis of such models could be carried out as a further extension of the Abelian analysis here.

\subsection{Singularities of $X_{n+1}$ and their global resolution}

In this section we determine all codimension two singularities of the elliptically fibered CYmanifold $X_{n+1}$. In general, the existence of rational sections in an elliptic fibration implies the presence of singularities in its Weierstrass model at codimension two in the base $B_{n}$ of the fibration, see e.g. [4, 9]. As stated above, we make the simplifying assumption that there are no codimension one singularities in the elliptic fibration. As we will demonstrate below, $X_{n+1}$ has Kodaira fibers of type $I_{2}$ at the codimension two loci in the base $B_{n}$ given in table 1 Here we have denoted the variety given by the vanishing locus of an ideal $I_{(k)}$ by $V\left(I_{(k)}\right), k=1, \ldots, 9$; we have also used the Weierstrass coordinates of the rational sections $\hat{s}_{Q}, \hat{s}_{R}$ given in (A.3), (A.4), and the shorthand notation

$$
\Delta_{i j}:=a_{i} b_{j}-b_{i} a_{j} .
$$

In addition, we specify the varieties $V\left(I_{(k)}\right), k=4, \ldots, 9$, in terms of reducible varieties from which we subtract unwanted irreducible components, employing $V(I \cdot J)=V(I) \cup V(J)$ for two ideals $I, J$. The corresponding prime ideals $I_{(k)}$ can be computed via the primary decomposition of the respective reducible variety. As discussed next, this analysis confirms 
that the list above gives the complete set of irreducible components of the $I_{2}$-loci. Further evidence of completeness is provided by anomaly cancellation in $6 \mathrm{D}$, which we show in section 4.2 .

Next, let us comment on the derivation of the codimension two loci in table 1. First, we recall that the presence of the two rational sections $\hat{s}_{Q}$ and $\hat{s}_{R}$ implies two factorizations of the Weierstrass form (3.11) of $X_{n+1}$. In the patch $z=1$, these factorizations read

$$
\left(y-\frac{y_{Q, R}}{z_{Q, R}^{3}}\right)\left(y+\frac{y_{Q, R}}{z_{Q, R}^{3}}\right)=\left(x-\frac{x_{Q, R}}{z_{Q, R}^{2}}\right)\left(x^{2}+\frac{x_{Q, R}}{z_{Q, R}^{2}} x+f+\frac{x_{Q, R}^{2}}{z_{Q, R}^{4}}\right),
$$

which implies a singularity at the following codimension two loci in the base $B_{n}$ :

$$
y_{Q, R}=0, \quad\left(z_{Q, R}\right)^{4} f+3\left(x_{Q, R}\right)^{2}=0 .
$$

The singularity at the loci (4.3) is of Kodaira type $I_{2}$, as can be read off by the vanishing orders of $(f, g, \Delta)$. These are precisely the two complete intersections entering the last two lines in table 1.

The locus (4.3) is a generically a reducible variety in $B_{n}$. Over each irreducible component the behavior of the three sections $\hat{s}_{P}, \hat{s}_{Q}$ and $\hat{s}_{R}$ is different, yielding different matter representations in F-theory. All irreducible matter loci are found by a decomposition of (4.3) into its prime ideals as employed in $[9,10]$. We claim that all its irreducible components are given in table 1. As this decomposition is in general computationally involved, it is necessary to identify a number of codimension two singularities of the WSF of $X_{n+1}$ by hand, as we demonstrate next.

\subsubsection{Singularities in the cubic fibration $X_{n+1}$}

The elliptic fibration $X_{n+1}$ in the form (3.4) is singular and admits conifold singularities. These can be made manifest by writing the defining equation (3.4) in the more suggestive form

$$
u f_{u}(u, v, w)+v_{1} v_{2} v_{3}=0
$$

where we defined

$$
f_{u}(u, v, w):=s_{1} u^{2}+s_{2} u v+s_{3} v^{2}+s_{5} u w+s_{6} v w+s_{8} w^{2}, \quad v_{i}:=a_{i} v+b_{i} w .
$$

This is a binomial geometry precisely of the form studied in [48, 49]. As discussed in these works there are three intersecting conifold singularities at

$$
u=f_{u}=0, \quad v_{i}=v_{j}=0(i<j),
$$

where we only consider the irreducible components of codimension two in $B_{n}$ of this variety. If $\left(a_{i}, b_{i}\right) \neq(0,0)$ and $\left(a_{j}, b_{j}\right) \neq 0$, this yields three codimension two loci

$$
\Delta_{i j}:=a_{i} b_{j}-b_{i} a_{j}=0, \quad s_{3} b_{i}^{2}-s_{6} a_{i} b_{i}+s_{8} a_{i}^{2}=0, \quad i=1,2,3,
$$

where the elliptic fiber is singular at $[u: v: w]=\left[0,-b_{i}, a_{i}\right]$. These are precisely the complete intersections entering the third to sixth lines of table 1. 
For the special values $\left(a_{i}, b_{i}\right)=(0,0)$ the second term in (4.4) vanishes. Thus. the elliptic fiber of (4.4) degenerates into the line $u=0$ and the conic $f_{u}=0$, which produces a resolved $I_{2}$ fiber at the three codimension two loci

$$
a_{i}=b_{i}=0, \quad i=1,2,3 .
$$

These three loci are the first to third lines in table 1 . Note that in these cases, the sections $\hat{s}_{P}, \hat{s}_{Q}$, and $\hat{s}_{R}$ all intersect the fiber along the line $u=0$; it is wrapped by that section whose coordinates are ill-defined at the respective locus in (4.8). Note also that while this $I_{2}$ singularity is resolved in the cubic model (3.4), the fiber is singular in the Weierstrass presentation.

There are three additional ways to factorize (4.4) into a line and a conic at codimension two in $B_{n}$. We write the factorization in the form

$$
\left(s_{1} u+a_{i} v+b_{i} w\right) q_{2}(u, v, w)=0, \quad i=1,2,3 .
$$

Here $q_{2}(u, v, w)=u^{2}+\ldots$ is a general conic in $u, v, w$. For generic $a_{i}, b_{i}$ the line $\left(s_{1} u+a_{i} v+b_{i} w\right)=0$ intersects the line $u=0$ at the single point $[u: v: w]=\left[0,-b_{i}, a_{i}\right]$, and precisely one of the rational points $P, Q, R$ is on it. The three different possibilities in (4.9) correspond to the three different choices of which rational point lies on the line. The codimension two locus in $B_{n}$ supporting these $I_{2}$ fibers can be computed using elimination ideals, see $[32,50]$, and is expected to yield the three ideals in the last three lines of table 1. Again, (4.9) is a resolved $I_{2}$ fiber in the cubic presentation of $X_{n+1}$.

\subsubsection{Global resolution of the cubic fibration $X_{n+1}$}

Thus, the only codimension two singularities of $X_{n+1}$ that require an additional resolution are the conifold singularities in (4.6). As shown in [48, 49], these are resolved by blowing up the ambient space $\mathbb{P}_{B}^{2}$ of (4.4) defined in (3.15) twice at $u=v_{i}=0$ and $f_{2}=v_{j}=0$, respectively. Introducing projective coordinates $\left[l_{1}: l_{2}\right],\left[m_{1}: m_{2}\right]$ on $\mathbb{F}_{0}=\mathbb{P}^{1} \times \mathbb{P}^{1}$, these two blow-ups are described by the complete intersection [51]

$$
l_{1} u-l_{2} v_{i}=0, \quad m_{1} f_{u}-m_{2} v_{j}=0 .
$$

For these two constraints to be well-defined sections of line bundles, the coordinates on $\mathbb{F}_{0}$ have to be sections of the following line bundles:

$$
l_{1} \in \mathcal{O}\left(\left[v_{i}\right]+H_{1}\right), \quad l_{2} \in \mathcal{O}\left([u]+H_{1}\right), \quad m_{1} \in \mathcal{O}\left(\left[v_{j}\right]+H_{2}\right), \quad m_{2} \in \mathcal{O}\left(\left[f_{u}\right]+H_{2}\right) .
$$

Here $H_{1}$ and $H_{2}$ denote the hyperplane classes of the two $\mathbb{P}^{1}$ 's in $\mathbb{F}_{0}$ and the divisor class associated to a section is denoted by [.] as before. The assignment (4.11) implies that $\mathbb{F}_{0}$ is fibered non-trivially over $\mathbb{P}_{B}^{2}$. Thus, the ambient space of the complete intersection (4.10) is the following $\mathbb{F}_{0}$-bundle:

$$
\mathbb{F}_{0} \longrightarrow \mathcal{W}=\mathbb{P}\left(\mathcal{O} \oplus \mathcal{O}\left([u]-\left[v_{i}\right]\right) \oplus \mathcal{O} \oplus \mathcal{O}\left(\left[f_{u}\right]-\left[v_{j}\right]\right)\right.
$$


Here the projectivization acts on the fiber coordinates in the four line bundle summands as the $\mathbb{C}^{*}$-actions in $\mathbb{F}_{0}$.

The proper transform of (4.4) in the blow-up (4.10) is readily computed using prime ideals. It agrees with the naive result one obtains by multiplying (4.4) by $l_{1} m_{1}$, using the two relations (4.10) and factoring out $v_{i} v_{j}$. In summary, we obtain the complete intersection

$$
l_{1} u-l_{2} v_{i}=0, \quad m_{1} f_{u}-m_{2} v_{j}=0, \quad l_{2} m_{2}+v_{k} l_{1} m_{1}=0, k \neq i, j
$$

in the ambient space (4.12) as the global resolution of (4.4). We denote this global resolution of $X_{n+1}$ as $\hat{X}_{n+1}$ in the following. A complete intersection resolution as (4.13) has also been used recently in [52] for the study of the $\mathbb{Z}_{3}$ Tate-Shafarevich group.

The Calabi-Yau condition of the complete intersection (4.13) is readily checked as follows. The first Chern class of (4.12) is computed as the sum of the first Chern class $c_{1}\left(\mathbb{P}_{B}^{2}\right)$ of its base $\mathbb{P}_{B}^{2}$ and the four fiber line bundles,

$$
\begin{aligned}
c_{1}(\mathcal{W}) & =c_{1}\left(\mathbb{P}_{B}^{2}\right)+[u]+\left[f_{u}\right]-\left[v_{i}\right]-\left[v_{j}\right]+2\left(H_{1}+H_{2}\right) \\
& =2\left([u]+\left[f_{u}\right]+H_{1}+H_{2}\right)-\left[v_{i}\right]-\left[v_{j}\right],
\end{aligned}
$$

where we have imposed the condition $c_{1}\left(\mathbb{P}_{B}^{2}\right)=[u]+\left[f_{u}\right]$ which is the Calabi-Yau condition of (4.4) in the ambient space (3.15) before resolution. Employing (4.11), we see that this is precisely the sum of the classes of the three constraints in (4.13), i.e. the complete intersection is indeed Calabi-Yau.

Next, we compute the coordinates of the rational points $P, Q$ and $R$ in the resolution $\hat{X}_{n+1}$. By plugging in their coordinates (3.5) into the complete intersection (4.13) we obtain their new coordinates in $\left[u: v: w: l_{1}: l_{2}: m_{1}: m_{2}\right]$ as

$$
\begin{aligned}
& P:\left[0:-b_{1}: a_{1}: f_{u}\left(0,-b_{1}, a_{1}\right): \Delta_{k 1} \Delta_{1 j}: \Delta_{j 1}: f_{u}\left(0,-b_{1}: a_{1}\right)\right] \\
& Q:\left[0:-b_{2}: a_{2}: f_{u}\left(0,-b_{2}, a_{2}\right): \Delta_{k 2} \Delta_{2 j}: \Delta_{j 2}: f_{u}\left(0,-b_{2}, a_{2}\right)\right] \\
& R:\left[0:-b_{3}: a_{3}: f_{u}\left(0,-b_{3}, a_{3}\right): \Delta_{k 3} \Delta_{3 j}: \Delta_{j 3}: f_{u}\left(0,-b_{3}, a_{3}\right)\right]
\end{aligned}
$$

where we used the shorthand notation $\Delta_{i j}=a_{i} b_{j}-b_{i} a_{j}$ defined in (4.7).

We note that we have obtained different resolutions, corresponding to the different choices of the labels $(i, j, k)$ in (4.13). For the purpose of computing the charged matter spectrum of F-theory, we only need one particular resolution of $X_{n+1}$. For convenience we choose $(i, j, k)=(1,2,3)$ in (4.13) yielding the resolution

$$
l_{1} u-l_{2} v_{1}=0, \quad m_{1} f_{u}-m_{2} v_{2}=0, \quad l_{2} m_{2}+v_{3} l_{1} m_{1}=0
$$

and the coordinates (4.15)

$$
\begin{aligned}
& P=\left[0:-b_{1}: a_{1}: f_{u}\left(0,-b_{1}, a_{1}\right): \Delta_{31} \Delta_{12}: \Delta_{21}: f_{u}\left(0,-b_{1}: a_{1}\right)\right], \\
& Q=\left[0:-b_{2}: a_{2}: 1: 0: 0: 1\right], \quad R=\left[0:-b_{3}: a_{3}: 1: 0: \Delta_{23}: f_{u}\left(0,-b_{3}, a_{3}\right)\right] .
\end{aligned}
$$

For latest results on the geometrical construction of different geometrical resolution phases, we refer e.g. to the recent works [53, 54]. 
We emphasize that the conifold singularities (4.6) are replaced by $\mathbb{P}^{1}$ 's, which are wrapped by rational points. The particular wrapping and the $\mathbb{P}^{1}$ inside $\mathbb{F}_{0}$ constituting the exceptional curve in $\hat{X}_{n+1}$ can be inferred from (4.16) and the coordinates (4.17) of the rational points. For example, at the original conifold locus $u=f_{u}=v_{1}=v_{2}=0$ in (4.6) the first and second equation in (4.16) are trivial so that $\left(l_{1}, l_{2}\right)$ and $\left(m_{1}, m_{2}\right)$ remain unconstrained. The third equation, that is of degree $(1,1)$ determines a diagonal $\mathbb{P}^{1}$ in $\mathbb{F}_{0}$ replacing the singularity. By (4.17) we see that it is wrapped by $P$, whose coordinates are ill-defined as $\Delta_{12}=f_{u}\left(0,-a_{1}, b_{1}\right)=0$.

We conclude the discussion of the resolution by noting that by Theorem 4.1 in [53] the complete intersection (4.13) is equivalent to

$$
l_{1} u-l_{2} v_{i}=0, \quad m_{1} v_{k}-m_{2} l_{2}=0, \quad m_{1} f_{u}+l_{1} m_{2} v_{j}=0, k \neq i, j .
$$

This describes two blow-ups, one at $u=v_{i}=0$, which is precisely the location of one rational point in $X_{n+1}$, and another at $v_{k}=l_{2}=0$, which is exactly the proper transform of a second rational point. The latter statement is clear since the first constraint in (4.13) implies that the set $\left\{l_{2}=v_{k}=0\right\}$ is equivalent to $\left\{u=v_{k}=0\right\}$ (except when $v_{k} \equiv v_{i}=0$ or $v_{k} \equiv v_{j}=0$ and $\left.f_{u}\right|_{u=v_{k}=0}=0$, where $l_{2}$ is unconstrained as we are at one conifold singularity in (4.6)). Thus, we see that the resolution (4.13) is nothing but an equivalent description of the blow-up at two rational points in $X_{n+1}$ given in (4.18).

\subsection{The matter spectrum}

In this section, we proceed to investigate the codimension two fibers in the resolution $\hat{X}_{n+1}$ constructed above. The charges of the hypermultiplets related to the isolated curves of the $I_{2}$ fibers are calculated in general from the intersection with the Shioda map of the sections, $\sigma\left(\hat{s}_{Q}\right)$ and $\sigma\left(\hat{s}_{R}\right)$, as $[4,55]$

$$
q_{1,2}=c \cdot \sigma\left(\hat{s}_{Q, R}\right)=c \cdot\left(S_{Q, R}-S_{P}\right),
$$

where $S_{P, Q, R}$ denotes the divisor class of the section $\hat{s}_{P, Q, R}$, respectively. We employ this formula and the particular resolution phase in (4.16) to compute the charged matter spectrum of the F-theory compactification on $X_{n+1}$.

\subsubsection{General model}

We begin by analyzing the resolution $\hat{X}_{n+1}$ of the elliptic fibration of the general model (3.4) at the nine codimension two loci in table 1 . In all cases, we obtain $I_{2}$ fibers, as discussed above, that enjoy different intersections with the rational sections $\hat{s}_{Q}, \hat{s}_{R}$ and the zero section $\hat{s}_{P}$. The resulting matter spectrum including $6 \mathrm{D}$ multiplicities $x_{\left(q_{1}, q_{2}\right)}$ of hypermultiplets with charges $\left(q_{1}, q_{2}\right)$ and a representation of the $I_{2}$ fibers in $\hat{X}_{n+1}$ in the phase (4.16) is shown in table 2 . The behaviour of the fiber is obtained by analyzing the complete intersection presentation (4.16) for $\hat{X}_{n+1}$ at all loci in table 1. In the figure, fiber components completely contained in one of the rational sections are shaded. We note that all fibers we find are compatible with the survey of possible $I_{2}$ fibers in [56]. 
The charges can be read off from the location of the rational sections on the fiber and (4.19), noting that the $\mathrm{U}(1)$ generators are associated with $Q-P$ and $R-P$. The fiber component not containing the zero point $P$ is the curve $c$ whose charges we compute. For example, in the first entry in table 2, the curve $c$ intersects twice with $P$ and not at all with $Q, R$, so the charges are $-2,-2$. We note that the matter spectrum is completely symmetric under exchange of the three points $P, Q$ and $R$ as expected by the symmetries of $X_{n+1}$ in the form (3.4). (Note that this symmetry is not manifest in table 1, though it is still there and can be seen by a different choice of generators of the respective ideals.)

The representation content in table 2 can be conveniently summarized by drawing the charge lattice of the theory. Since a $6 \mathrm{D}$ hypermultiplet contains fields both in a representation and its complex conjugate, we draw all the charges in table 2 including their negative, yielding precisely the charge lattice that was depicted in figure 4 . Thus, all the generic models have spectra that appear compatible with a Higgsed $\mathrm{SU}(2) \times \mathrm{SU}(2) \times \mathrm{SU}(3)$ model, with symmetric SU(3) matter included; we see in the following section how this works in detail.

The multiplicities of the matter representations in table 2 are given by the number of points in the base $B_{n}$ supporting a given $I_{2}$ fiber in $\hat{X}_{n+1}$. The multiplicities of the hypermultiplets in the first three lines of table 2 readily follow from table 1 and the line bundles in (3.17). The multiplicities in the fourth to sixth lines are computed from table 1 starting with the corresponding reducible complete intersection and subtracting the respective irreducible component $a_{i}=b_{i}=0$ which is contained in it with the appropriate multiplicity, that is calculated to be two using the resultant (see [9] for more details on this method). For example, for the matter with charge $(-2,-1)$, we compute the resultant of $\Delta_{12}$ and $s_{3} b_{1}^{2}-s_{6} a_{1} b_{1}+s_{8} a_{1}^{2}$ w.r.t. $a_{1}$. It has a factor of $b_{1}^{2}$, indicating that $a_{1}=b_{1}=0$ is a zero of order two as claimed. Thus we obtain

$$
\begin{aligned}
x_{(-2,-1)} & =\left(2\left[a_{1}\right]+\left[s_{8}\right]\right) \cdot\left(\left[a_{1}\right]+\left[a_{2}\right]+\left[s_{8}\right]-\left[K_{B}^{-1}\right]\right)-2 x_{(2,2)}, \\
x_{(-1,-2)} & =\left(2\left[a_{3}\right]+\left[s_{8}\right]\right) \cdot\left(\left[a_{1}\right]+\left[a_{3}\right]+\left[s_{8}\right]-\left[K_{B}^{-1}\right]\right)-2 x_{(0,2)}, \\
x_{(-1,1)} & =\left(2\left[a_{2}\right]+\left[s_{8}\right]\right) \cdot\left(\left[a_{2}\right]+\left[a_{3}\right]+\left[s_{8}\right]-\left[K_{B}^{-1}\right]\right)-2 x_{(2,0)},
\end{aligned}
$$

which precisely reproduces the fourth to sixth line of table 2 employing (3.17).

For the computation of the multiplicity $x_{(1,1)}$ of the hypermultiplets with charges $(1,1)$, we instead use the ideal (4.19) in [9], that is $\left\langle g_{9}^{\prime}, \delta g_{6}\right\rangle$, with the coefficients $s_{i} \rightarrow \tilde{s}_{i}$ according to (3.13). ${ }^{8}$ We calculate $x_{(1,1)}$ by subtracting unwanted irreducible components with their appropriate multiplicities. These are computed using the resultant as outlined before. Due to computational limitations we have to use random numbers for those coefficients $s_{i}, a_{j}$, $b_{j}$ which are not the variables on which the resultant depends. We obtain

$$
\begin{aligned}
x_{(1,1)} & =\left(\left[g_{9}^{\prime}\right] \cdot\left[\delta g_{6}\right]\right)_{s \rightarrow \tilde{s}}-2 x_{(2,0)}-8 x_{(-2,-1)}-4 x_{(-1,-2)}-20 x_{(-2,-2)}, \\
& =\left(\left[a_{1}^{4} a_{2}^{2} s_{8}^{3}\right]\right) \cdot\left(\left[a_{1}^{4} a_{2} b_{3} s_{8}^{2}\right]\right)-2 x_{(2,0)}-8 x_{(-2,-1)}-4 x_{(-1,-2)}-20 x_{(-2,-2)},
\end{aligned}
$$

which yields precisely line seven in table 2 using (3.17).

\footnotetext{
${ }^{8}$ It is advantageous to use this ideal because it already has some components subtracted.
} 


\begin{tabular}{|c|c|c|}
\hline Charges & Multiplicity & Fiber \\
\hline$(-2,-2)$ & $x_{(-2,-2)}=\left[a_{1}\right] \cdot\left(\left[a_{1}\right]+\left[s_{8}\right]+K_{B}\right)$ & \\
\hline$(2,0)$ & $x_{(2,0)}=\left[a_{2}\right] \cdot\left(\left[a_{2}\right]+\left[s_{8}\right]+K_{B}\right)$ & \\
\hline$(0,2)$ & $x_{(0,2)}=\left[a_{3}\right] \cdot\left(\left[a_{3}\right]+\left[s_{8}\right]+K_{B}\right)$ & \\
\hline$(-2,-1)$ & $x_{(-2,-1)}=\left[s_{8}\right] \cdot\left(\left[s_{8}\right]+\left[a_{1}\right]+\left[a_{2}\right]+K_{B}\right)+2\left[a_{1}\right] \cdot\left[a_{2}\right]$ & \\
\hline$(-1,-2)$ & $x_{(-1,-2)}=\left[s_{8}\right] \cdot\left(\left[s_{8}\right]+\left[a_{1}\right]+\left[a_{3}\right]+K_{B}\right)+2\left[a_{1}\right] \cdot\left[a_{3}\right]$ & \\
\hline$(-1,1)$ & $x_{(-1,1)}=\left[s_{8}\right] \cdot\left(\left[s_{8}\right]+\left[a_{2}\right]+\left[a_{3}\right]+K_{B}\right)+2\left[a_{2}\right] \cdot\left[a_{3}\right]$ & \\
\hline$(1,1)$ & $\begin{array}{c}x_{(1,1)}=-K_{B} \cdot\left(16\left[a_{1}\right]+9\left[s_{8}\right]\right)-4\left[a_{1}\right] \cdot\left(\left[a_{1}\right]+\left[a_{2}\right]\right) \\
+2\left[a_{3}\right] \cdot\left(\left[a_{2}\right]-2\left[a_{1}\right]\right)-\left(8\left[a_{1}\right]+\left[a_{2}\right]+\left[a_{3}\right]\right) \cdot\left[s_{8}\right]-3\left[s_{8}\right]^{2}\end{array}$ & \\
\hline$(1,0)$ & $\begin{array}{c}x_{(1,0)}=-K_{B} \cdot\left(16\left[a_{2}\right]+9\left[s_{8}\right]\right)-4\left[a_{2}\right] \cdot\left(\left[a_{2}\right]+\left[a_{3}\right]\right) \\
+2\left[a_{1}\right] \cdot\left(\left[a_{3}\right]-2\left[a_{2}\right]\right)-\left(\left[a_{1}\right]+8\left[a_{2}\right]+\left[a_{3}\right]\right) \cdot\left[s_{8}\right]-3\left[s_{8}\right]^{2}\end{array}$ & \\
\hline$(0,1)$ & $\begin{array}{c}x_{(0,1)}=-K_{B} \cdot\left(16\left[a_{3}\right]+9\left[s_{8}\right]\right)-4\left[a_{3}\right] \cdot\left(\left[a_{2}\right]+\left[a_{3}\right]\right) \\
+2\left[a_{1}\right] \cdot\left(\left[a_{2}\right]-2\left[a_{3}\right]\right)-\left(\left[a_{1}\right]+\left[a_{2}\right]+8\left[a_{3}\right]\right) \cdot\left[s_{8}\right]-3\left[s_{8}\right]^{2}\end{array}$ & \\
\hline
\end{tabular}

Table 2. Matter spectrum and corresponding $I_{2}$-fibers in $\hat{X}_{n+1}$. 
Finally, the multiplicities of the hypermultiplets with charges $(1,0)$ and $(0,1)$ are calculated from the ideals in the last two lines of table 2 after subtracting, with the right degrees, the components corresponding to the other charged hypermultiplets. The multiplicities of the hypermultiplets with charges $(1,0)$ and $(0,1)$ are

$$
x_{(1,0)}=\left[y_{Q}\right] \cdot\left[z_{Q}^{4} f+3 x_{Q}^{2}\right]-16 x_{(2,0)}-16 x_{(-2,-1)}-x_{(-1,-2)}-16 x_{(-2,-2)}-x_{(-1,1)}-x_{(1,1)}
$$

and

$$
x_{(0,1)}=\left[y_{R}\right] \cdot\left[z_{R}^{4} f+3 x_{R}^{2}\right]-x_{(-2,-1)}-16 x_{(0,2)}-16 x_{(-1,-2)}-16 x_{(-2,-2)}-x_{(-1,1)}-x_{(1,1)},
$$

where the latter can also be obtained from $x_{(1,0)}$ using the symmetry $Q \leftrightarrow R$, as expected. Employing (3.17), we obtain the last two lines of table 2.

We conclude by discussing anomaly cancellation in $6 \mathrm{D}$. Using the charges and their respective multiplicities in table 2 , we proceed to calculate the anomalies of the Abelian theory following [36, 37] (we omit the details of this calculation). The only piece of information missing are the coefficients $b_{m n}$ of the Green-Schwarz counterterms. They are calculated by the Neron-Tate height pairing as

$$
b_{m n}=-\pi\left(\sigma\left(\hat{s}_{m}\right) \cdot \sigma\left(\hat{s}_{n}\right)\right)
$$

where $\sigma$ is the Shioda map which maps sections to elements in $H^{(1,1)}\left(\hat{X}_{n+1}\right)[4,55,57]$, and the map $\pi$ is the projection operator to homology $H^{(1,1)}\left(B_{n}\right)$ of the base. The Shioda map for a section $\hat{s}_{m}$ with divisor class $S_{m}$ reads explicitly

$$
\sigma\left(\hat{s}_{m}\right)=S_{m}-S_{P}-\left[K_{B}^{-1}\right]-\pi\left(S_{m} \cdot S_{P}\right),
$$

thus, the Green-Schwarz counterterms are

$$
b_{m n}=\left(\begin{array}{cc}
2\left(\pi\left(S_{Q} \cdot S_{P}\right)-K_{B}\right) & \pi\left(S_{Q} \cdot S_{P}+S_{R} \cdot S_{P}-S_{Q} \cdot S_{R}\right)-K_{B} \\
\pi\left(S_{Q} \cdot S_{P}+S_{R} \cdot S_{P}-S_{Q} \cdot S_{R}\right)-K_{B} & 2\left(\pi\left(S_{R} \cdot S_{P}\right)-K_{B}\right)
\end{array}\right)_{m n}
$$

The divisor classes $\pi\left(S_{m} \cdot S_{n}\right)$ are precisely the classes of the respective constraint in (3.6), which are just the classes of $\Delta_{i j}$ defined in (4.1). Using (3.17), we obtain the following Neron-Tate height pairing matrix:

$$
b_{m n}=\left(\begin{array}{cc}
2\left(\left[a_{1}\right]+\left[b_{2}\right]+\left[s_{8}\right]\right) & 2\left[a_{1}\right]+\left[s_{8}\right] \\
2\left[a_{1}\right]+\left[s_{8}\right] & 2\left(\left[a_{1}\right]+\left[a_{3}\right]+\left[s_{8}\right]\right)
\end{array}\right)_{m n} .
$$

Finally, using the full spectrum and the coefficients $b_{m n}$, we confirm that all anomalies are canceled by a generalized Green-Schwarz mechanism in 6D (refer e.g. to [36, 37] for a review on Abelian anomalies).

\subsubsection{Specialized model}

Next, we turn to the specialized model defined by (3.8). Its $I_{2}$ fibers and spectrum of charged matter fields are obtained directly from table 1 and table 2 , respectively, by setting 


\begin{tabular}{|c|c|}
\hline Charges & Multiplicity \\
\hline$(2,0)$ & $x_{(2,0)}=\left[a_{2}\right] \cdot\left(\left[a_{2}\right]+\left[s_{8}\right]+K_{B}\right)$ \\
\hline$(0,2)$ & $x_{(0,2)}=\left[a_{3}\right] \cdot\left(\left[a_{3}\right]+\left[s_{8}\right]+K_{B}\right)$ \\
\hline$(-2,-1)$ & $x_{(-2,-1)}=\left[s_{8}\right] \cdot\left(\left[a_{2}\right]+\left[s_{8}\right]+K_{B}\right)$ \\
\hline$(-1,-2)$ & $x_{(-1,-2)}=\left[s_{8}\right] \cdot\left(\left[a_{3}\right]+\left[s_{8}\right]+K_{B}\right)$ \\
\hline$(-1,1)$ & $x_{(-1,1)}=\left[s_{8}\right] \cdot\left(\left[a_{3}\right]+\left[s_{8}\right]+K_{B}\right)+\left[a_{2}\right] \cdot\left(2\left[a_{3}\right]+\left[s_{8}\right]\right)$ \\
\hline$(1,1)$ & $\begin{array}{l}x_{(1,1)}=-9 K_{B} \cdot\left[s_{8}\right]+2\left[a_{2}\right] \cdot\left[a_{3}\right] \\
\quad-\left(\left[a_{2}\right]+\left[a_{3}\right]\right) \cdot\left[s_{8}\right]-3\left[s_{8}\right]^{2}\end{array}$ \\
\hline$(1,0)$ & $\begin{array}{c}x_{(1,0)}=-K_{B} \cdot\left(16\left[a_{2}\right]+9\left[s_{8}\right]\right)-4\left[a_{2}\right] \cdot\left(\left[a_{2}\right]+\left[a_{3}\right]\right) \\
-\left(8\left[a_{2}\right]+\left[a_{3}\right]\right) \cdot\left[s_{8}\right]-3\left[s_{8}\right]^{2}\end{array}$ \\
\hline$(0,1)$ & $\begin{array}{c}x_{(0,1)}=-K_{B} \cdot\left(16\left[a_{3}\right]+9\left[s_{8}\right]\right)-4\left[a_{3}\right] \cdot\left(\left[a_{2}\right]+\left[a_{3}\right]\right) \\
-\left(\left[a_{2}\right]+8\left[a_{3}\right]\right) \cdot\left[s_{8}\right]-3\left[s_{8}\right]^{2}\end{array}$ \\
\hline
\end{tabular}

Table 3. Matter spectrum of the simplified model $\hat{X}_{n+1}$ with $a_{1}=1$ and $b_{1}=0$.

$a_{1}=1, b_{1}=0$. We obtain the results summarized in table 3 . We note that the matter fields with charge $(-2,-2)$ in table 2 are not present due to $\left[a_{1}\right]=0$. Again, we can summarize the spectrum in table 3 by drawing the charge lattice of the theory, giving the spectrum shown in figure 3 , corresponding to a Higgsed $\mathrm{SU}(2) \times \mathrm{SU}(2) \times \mathrm{SU}(3)$ model without symmetric $\mathrm{SU}(3)$ matter.

Anomaly cancellation can be readily checked using the spectrum in table 3 and using the anomaly coefficients $b_{m n}$ in (4.28) for $\left[a_{1}\right]=0$.

\subsection{Comparison with matter in $d P_{2}$-elliptic fibrations}

We conclude by continuing the discussion of section 3.4 on the connection between the models $X_{n+1}$ and $d P_{2}$-elliptic fibrations, now at the level of the matter spectrum.

First, we recall that $a_{1}$ and $b_{2}$ have to be non-vanishing constants in a $d P_{2}$-elliptic fibration. Thus, the representations with charges $(2,0),(-2,-1)$ and $(-2,-2)$ in table 2 are not realized. These are the representations related to the collision of the sections $\hat{s}_{Q}$ and $\hat{s}_{P}$, which, however, is impossible for an elliptic fiber in $d P_{2}$. Drawing all the charges in a two-dimensional lattice yields figure 7 . A quick glance at this picture makes it clear why a complete unHiggsing of the $d P_{2}$-model to the gauge groups $\mathrm{SU}(2) \times \mathrm{SU}(2)$ and $\mathrm{SU}(3)$ with more than one adjoint is not feasible: the representations $\pm(2,0)$ and $\pm(2,1)$, which come from the $\mathrm{SU}(2)$ or $\mathrm{SU}(3)$ adjoints, respectively, are not present.

As mentioned in section 3.4, there are $d P_{2}$-models which are not accessible from specializations of $X_{n+1}$, as the effectiveness constraints in the latter models are more constraining than in the former. To see this, let us set the classes $\left[a_{1}\right]=0$ and $\left[b_{2}\right]=0$ in (3.19). Once these constraints are imposed, the effectiveness condition $\left[b_{1}\right] \geq 0$ and $\left[a_{2}\right] \geq 0$ automatically forces the constraints $\left[s_{8}\right]=-K_{B},\left[a_{2}\right]=0$ and $\left[b_{1}\right]=0$, restricting the set of possible models to a one-parameter family parametrized by the degree of $\left[a_{3}\right]$. However, it has been shown in [9-12] that a $d P_{2}$-elliptic fibration is parametrized by two divisor classes. 


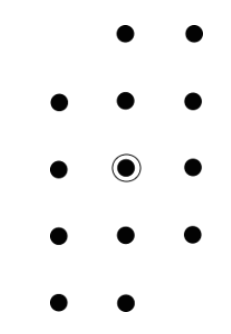

Figure 7. Weight lattice of charges of the $\mathrm{U}(1) \times \mathrm{U}(1)$ theory obtained from $d P_{2}$-elliptic fibrations. The origin of the lattice is indicated by a circle.

Thus, we only obtain a subset of models by specializing $X_{n+1}$. These models, however, admit unHiggsings to $\mathrm{SU}(3) \times \mathrm{SU}(2)$ on $s_{8}=0$ and $a_{3}=0$, respectively, along the lines of section 5 .

More concretely, specific examples of $d P_{2}$-fibrations over $\mathbb{P}^{2}$ and $\mathbb{P}^{3}$ were listed in figure 2 in [10] and table 2.3 in [11]. In those cases it was shown that the complex structure moduli space is stratified, with integral points in a two-dimensional polygon corresponding to the different strata. Let us compare the models obtained from $X_{n+1}$ through the specialization $\left[a_{1}\right]=0$ and $\left[b_{2}\right]=0$, with the models obtained from $d P_{2}$-fibrations over $\mathbb{P}^{2}$. Imposing these specializations in the Calabi-Yau manifolds $X_{n+1}$, we obtain only five different models labelled by the different values of $\left[a_{3}\right]$ :

\begin{tabular}{|c|c||c|c|c|c|c|c|c|c|}
\hline$\left[a_{2}\right]$ & {$\left[a_{3}\right]$} & $b_{2}$ & $b_{3}$ & $s_{1}$ & $s_{2}$ & $s_{3}$ & $s_{5}$ & $s_{6}$ & $s_{8}$ \\
\hline 0 & 0 & 0 & 0 & 9 & 6 & 3 & 6 & 3 & 3 \\
0 & 1 & 0 & 1 & 7 & 5 & 3 & 5 & 3 & 3 \\
0 & 2 & 0 & 2 & 5 & 4 & 3 & 4 & 3 & 3 \\
0 & 3 & 0 & 3 & 3 & 3 & 3 & 3 & 3 & 3 \\
0 & 4 & 0 & 4 & 1 & 2 & 3 & 2 & 3 & 3 \\
\hline
\end{tabular}

In contrast, there are $31 d P_{2}$-elliptic fibrations over $\mathbb{P}^{2}$. We can draw all the models in a two-dimensional diagram, with axes given by the values of $n_{a_{3}}$ and $n_{b_{3}}$ where we expanded $\left[a_{3}\right]:=n_{a_{3}} H_{B}$ and $\left[b_{3}\right]=n_{b_{3}} H_{B}$ with $H_{B}$ the hyperplane in $\mathbb{P}^{2} .{ }^{9}$ We obtain figure 8 . As indicated here, only the subset of models on the diagonal is described by models $X_{n+1}$ with $\left[a_{1}\right]=\left[b_{2}\right]=0$.

\section{UnHigssing two U(1)'s in F-theory}

In this section, we discuss the unHiggsing of Abelian to non-Abelian gauge symmetries for the case of two $\mathrm{U}(1)$ factors. We distinguish between rank-preserving unHiggsings to non-Abelian groups with adjoints and unHiggsing with rank enhancement to non-Abelian groups that do not necessarily have adjoints. In the latter case, there are situations when the additional non-Abelian groups are spectators to a rank-preserving unHiggsing of the $\mathrm{U}(1)$ 's and other situations when the additional groups are involved in the unHiggsing of the Abelian theory.

\footnotetext{
${ }^{9}$ The parameters $s_{7}$ and $s_{9}$ in $[9,10]$ are identified with $a_{3}, b_{3}$ in $X_{n+1}$, respectively.
} 


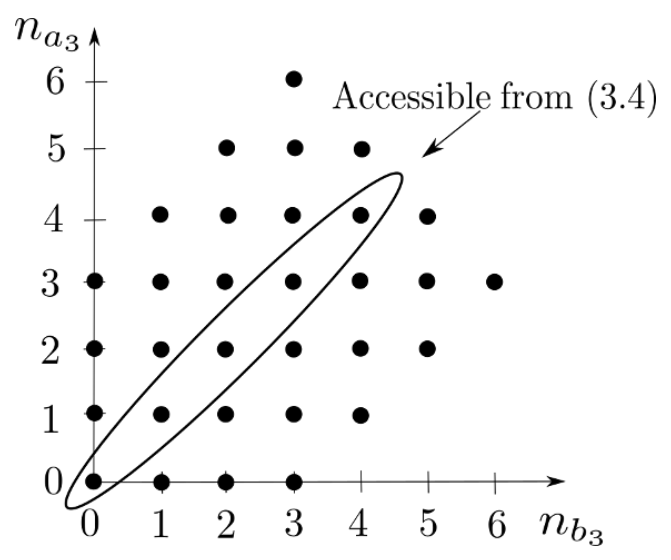

Figure 8. Region of allowed $d P_{2}$ fibrations over $\mathbb{P}^{2}$. The set of models obtainable by specializing (3.4) is given by the five encircled points.

We first discuss the general geometrical unHiggsing procedure in section 5.1. Then we apply this to the particular case of our Abelian model $X_{n+1}$ in sections 5.2, 5.3 and 5.6 and discover an unHiggsing to a model with $\mathrm{SU}(2) \times \mathrm{SU}(2) \times \mathrm{SU}(3)$ gauge group and no $\mathrm{U}(1)$ 's. In section 5.4 we analyze its Weierstrass form which we use in section 4.2 to derive the full matter spectrum. We put special emphasis on the emergence of the symmetric representation 6 of $\mathrm{SU}(3)$. In section 5.7 we relate the appearance of this new matter representation to an ordinary double point singularity on the $\mathrm{SU}(3)$ divisor which can not be deformed,

\subsection{UnHiggsing in the complex structure moduli space}

We begin with a discussion of the general geometrical procedure underlying the unHiggsing of a $\mathrm{U}(1)$ gauge symmetry in F-theory. We put special emphasis on rank-preserving unHiggsings because they have a clear geometric interpretation as a transition of a rational section to a Cartan divisor.

The unHiggsing process of an F-theory compactification with $\mathrm{U}(1)^{k}$ gauge symmetry can be understood geometrically as a transition from one Calabi-Yau manifold $X_{n+1}^{(k)}$ with a rank $k$ MW-group to another Calabi-Yau manifold $X_{n+1}^{\left(k^{\prime}\right)}$ with a lower rank MW-group, $k^{\prime}<k$. The manifold $X_{n+1}^{\left(k^{\prime}\right)}$ is reached via a tuning of the complex structure of $X_{n+1}^{(k)}$ so that two rational sections are placed on top of each other, i.e. define maps from the base $B_{n}$ to the same point in the elliptic fiber $\mathcal{E}$. In this process certain codimension two singularities, typically of Kodaira type $I_{2}$, that the fibration of $X_{n+1}^{(k)}$ exhibits as a consequence of the presence of U(1)'s and that give rise to U(1)-charged matter in F-theory (cf. section 4) are promoted to codimension one singularities of $X_{n+1}^{(k-1)}$. Repeating such tunings of the complex structure until all sections are placed on top of each other we eventually obtain a Calabi-Yau manifold $X_{n+1}^{(0)}$ with trivial MW-group, i.e. no abelian gauge group factors.

The structure of the codimension one singularities of the elliptic fibration of $X_{n+1}^{(0)}$ encodes the original Abelian gauge theory of F-theory on $X_{n+1}^{(k)}$ in terms of a non-Abelian gauge theory with gauge group $G$ and a specific matter spectrum. The case of a single 
$\mathrm{U}(1)$ has been analyzed in [14] and is reviewed in section 2.1. It has been shown that every model with a $\mathrm{U}(1)$ can be unHiggsed to a model with $\mathrm{SU}(2)$ or larger non-Abelian gauge group, although in some cases, particularly when there are additional non-Abelian factors present before the unHiggsing, the unHiggsed model can develop singularities. UnHiggsing of toric models with up to three $\mathrm{U}(1)$ 's to $\left(\mathrm{SU}(2)^{2} \times \mathrm{SU}(4)\right) / \mathbb{Z}_{2}$ and $\mathrm{SU}(3)^{3} / \mathbb{Z}_{3}$ have been found in [32]. In general, one naturally expects that the minimal rank of the gauge group $G$ is at least $k$, so that by Higgsing e.g. via adjoints we can recover the full Abelian gauge group $\mathrm{U}(1)^{k}$. However, for some abelian theories the rank of $G$ has to be larger depending on the matter spectrum of the original Abelian theory. Theories with a sufficiently simple spectrum unHiggs to a group $G$ of rank $k$, where the Higgs is in the adjoint, whereas theories with a more complex spectrum including matter of higher charges require $G$ of rank greater than $k$ and Higgses in other representations, e.g. the (bi-)fundamental.

The preceding discussion is general and thus should apply to elliptic fibrations with an arbitrary number of rational sections. For the rest of this section, however, we work explicitly in the specific context of the three section fibration $X_{n+1}$ defined by (3.4), i.e. we identify $X_{n+1}^{(2)} \equiv X_{n+1}$. We establish the following picture for unHiggsing F-theory models with $\mathrm{U}(1)^{2}$ gauge symmetry obtained from these general elliptic fibrations $X_{n+1}$. In agreement with the expectation from field theory, cf. section 2 , we demonstrate that, indeed, for sufficiently simple Abelian models the minimal rank of $G$ is $k=2$. This leads to two natural classes of models that admit a rank-preserving unHiggsing of $\mathrm{U}(1)^{2}$ either to $\mathrm{SU}(2) \times \mathrm{SU}(2)$ or to $\mathrm{SU}(3)$ on base divisors supporting adjoints, i.e. divisors in classes of the form $-K_{B}+Z$ for an effective divisor $Z$ in $B_{n}$. There are, however, many models with $\mathrm{U}(1)^{2}$ gauge group that due to the complexity of their matter spectrum require the introduction of additional non-Abelian groups in the unHiggsed geometry; the resulting non-Abelian models, typically have rank-reducing Higgses in (bi-)fundamental representations.

We demonstrate furthermore, in accord with the discussion of section 2, that the nonAbelian gauge symmetry underlying a large class of models with $\mathrm{U}(1)^{2}$ gauge symmetry realized via $X_{n+1}^{(2)}$ via a complete unHiggsing is given by

$$
G_{\text {uni }}=\mathrm{SU}(2) \times \mathrm{SU}(2) \times \mathrm{SU}(3) .
$$

The minimal non-Abelian gauge group can be even larger, as in the case of a single U(1), in degenerate cases where the non-Abelian factors can be thought of as living on reducible divisors. We also show that in some cases the unHiggsing is not unique and multiple different gauge groups $G$ can be obtained, some of which can exceed $G_{\text {uni }}$ even if others do not.

\subsection{Geometrical unHiggsing of one of the U(1)'s}

Following the discussion of the previous subsection, we wish to unHiggs the $\mathrm{U}(1)^{2}$ gauge group of F-theory compactified on $X_{n+1}$ by reducing its Mordell-Weil group through an appropriate tuning of its complex structure.

Given the elliptically fibered CY-manifold $X_{n+1}$ in the form (3.4), we recall that two of its rational sections coincide if the coefficients $a_{i}, b_{i}$ are chosen so that one of the constraints 


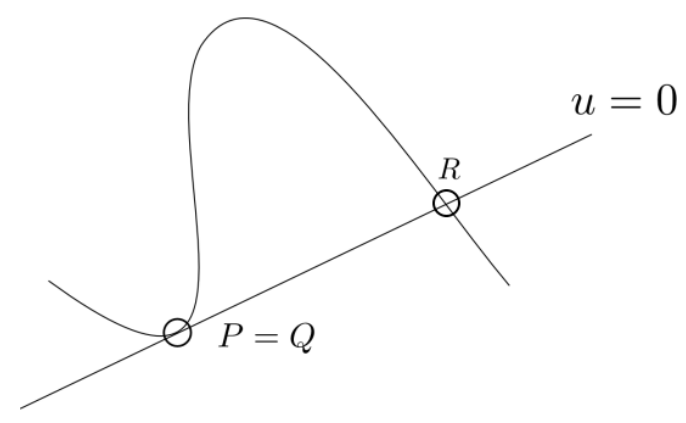

Figure 9. Tuned elliptic curve $\mathcal{E}$ with $P=Q$ yielding an elliptic fibration with rank one MordellWeil group.

in the first line of (3.6) is obeyed. For instance, in order to achieve $\hat{s}_{P}=\hat{s}_{Q}$ we have to demand $P=Q$ in the elliptic fiber $\mathcal{E}$ as shown in figure 9 . This is achieved by choosing the complex structure of $X_{n+1}$ such that

$$
\Delta_{12}=a_{1} b_{2}-a_{2} b_{1} \equiv 0,
$$

where we have used the definition (4.1).

There are a number of ways in which (5.2) may be realized. We list some of the main possibilities:

A) One simple way in which (5.2) may be satisfied is if the divisor class $\left[a_{2}\right]-\left[a_{1}\right]$ is effective. In this case, we can introduce a section $\lambda_{1}$ of the line bundle

$$
\lambda_{1} \in \mathcal{O}\left(\left[a_{2}\right]-\left[a_{1}\right]\right),
$$

and impose the condition

$$
\left(a_{2} v+b_{2} w\right) \stackrel{!}{=} \lambda_{1}\left(a_{1} v+b_{1} w\right) .
$$

We then have $a_{2}=\lambda_{1} a_{1}, b_{2}=\lambda_{1} b_{1}$ and (5.2) is satisfied. This possibility is available in many of the simplest cases, such as when $\left[a_{1}\right]=0$ (then we can also shift $v$ so that $b_{1}=0$; the solution to $(5.2)$ is then $\left.b_{2}=0\right)$, or for example on the base $\mathbb{P}^{2}$, where all divisors are multiples of the line $H_{B}$ so we can simply order $\left[a_{2}\right]>\left[a_{1}\right]$. As we show explicitly in the next section, this choice of tuning leads to an unHiggsing of the $\mathrm{U}(1)$ associated to $\hat{s}_{Q}$ to a non-Abelian group $\mathrm{SU}(2) \times \mathrm{SU}(2)$. This leads directly to a general unHiggsing of the two U(1) model that matches nicely with the field-theoretic discussion of section 2

B) In some situations, neither $\left[a_{2}\right]-\left[a_{1}\right]$ or $\left[a_{1}\right]-\left[a_{2}\right]$ is effective, but the ring of functions/sections is a UFD, so that we can solve (5.2) by taking

$$
a_{1}=A B, b_{2}=C D, a_{2}=A C, b_{1}=B D .
$$

In this situation we again get an unHiggsing of one $\mathrm{U}(1)$ to $\mathrm{SU}(2) \times \mathrm{SU}(2)$, on the divisors $B, C$. We do not explore the details of this unHiggsing here. 
C) Even if the ring is not a UFD, there can be situations where (5.2) can again be solved for nonzero functions $a_{1}, a_{2}, b_{1}, b_{2}$. This unHiggsing gives only a single $\mathrm{SU}(2)$ on $f_{u}\left(0,-b_{1}, a_{1}\right)=0$ with $f_{u}$ defined in (4.5).

D) Finally, there are situations where there are no solutions of (5.2) for $a_{1}, a_{2}, b_{1}, b_{2}$ all nonzero. For example, if the base is $d P_{3}$, where the cone of effective curves is generated by three exceptional curves $E_{1}, E_{2}, E_{3}$ from blowing up three points on $\mathbb{P}^{2}$, and three lines $L_{12}, L_{23}, L_{13}$ that connect the points, we may have $\left[a_{1}\right]=E_{1},\left[b_{2}\right]=$ $L_{12},\left[a_{2}\right]=L_{23},\left[b_{1}\right]=E_{3}$. In this case even though the divisor classes are equivalent, $\left[a_{1} b_{2}\right]=\left[a_{2} b_{1}\right]$, there is no way to solve (5.2), since e.g. $a_{1}$ must vanish on the rigid curve $E_{1}$, but $a_{2}, b_{1}$ cannot.

Even in this type of situation, however, there is a solution of (5.2), which is in fact available for all possible bases and choices of $\left[a_{i}\right],\left[b_{i}\right]$. We can set e.g. $b_{2}=b_{1}=0$, which immediately leads to a solution of (5.2). While this tuning can be carried out in any of the possible $\mathrm{U}(1)^{2}$ models, even when the tuning of type (A) above is not available, we can relate this to a special case of the tuning (A) by taking the tuning in two steps. First, we can tune $b_{1}=0$. This sets $v_{1}=a_{1} v$, so that the equation

$$
u f_{u}=a_{1} v v_{2} v_{3}
$$

develops an $I_{2}$ singularity on the locus $a_{1}=0$. We may then, however, move the factor of $a_{1}$ into $v_{2}$, defining a new model with $\tilde{a}_{2}=a_{1} a_{2}, \tilde{b}_{2}=a_{1} b_{2}$ and $\tilde{a}_{1}=1$. This then becomes a special case of a geometry where (A) is available, since $\tilde{a}_{1}=1$, though the UnHiggsing through (A) leads to an extra $\mathrm{SU}(2)$ factor since $\tilde{a}_{2}$ is a reducible polynomial.

In the remainder of this section we focus on approach (A) to unHiggsing, since not only is it relevant in the clearest and simplest cases, but through the mechanism just mentioned it also covers the completely general case (D), albeit at the cost of producing an additional $\mathrm{SU}(2)$ factor due to reduciblity of $\tilde{a}_{2}$.

\subsection{Unhiggsing both U(1)'s}

We now describe the process of unHiggsing both $\mathrm{U}(1)$ factors in a situation where approach (A) can be done in both cases. This is the situation, for example, on the base $\mathbb{P}^{2}$, or if one of the $a_{i}$ is in the trivial class $\left[a_{1}\right]=0$. More generally, it is possible to do this whenever $\left[a_{2}\right]-\left[a_{1}\right]$ and $\left[a_{3}\right]-\left[a_{1}\right]$ are both effective. In a general situation where a direct application of $(\mathrm{A})$ is not possible in both cases, as discussed above we can unHiggs by first setting $b_{1}=0$ and then considering two applications of $(\mathrm{A})$, using the redefinitions

$$
\tilde{a}_{1}=1, \tilde{a}_{2}=a_{1} a_{2}
$$

where $\tilde{a}_{2}$ is a reducible divisor. In the discussion here we assume that the direct application of (A) can proceed in both cases; other situations such as those where only the unHiggsing approach (D) is available are special cases where one of the divisors $a_{i}$ becomes degenerate. 
As discussed above, we begin by solving (5.2) by setting $v_{2}=\lambda_{1} v_{1}$. Plugging this into the CY-equation (3.4) for $X_{n+1}$ we obtain

$$
p=u f_{u}(u, v, w)+\lambda_{1}\left(a_{1} v+b_{1} w\right)^{2}\left(a_{3} v+b_{3} w\right),
$$

where the polynomial $f_{u}$ is defined in (4.5).

Clearly, this tuned elliptic fibration is singular at codimension one. We immediately observe the $I_{2}$ fiber $u f_{u}=0$, corresponding to an $\mathrm{SU}(2)$ gauge group in F-theory, at the divisor $\lambda_{1}=0$ in $B_{n}$, which we simply denote by $\lambda_{1}$. It is in the class

$$
\mathrm{SU}(2): \quad\left[\lambda_{1}\right]=\left[a_{2}\right]-\left[a_{1}\right] .
$$

This $I_{2}$ fiber is already resolved in the $\mathbb{P}^{2}$ model (3.4). In addition, (5.8) is the equation of an (unresolved) $A_{1}$-singularity at $u=f_{u}=a_{1} v+b_{1} w=0$, i.e. a Kodaira singularity of type $I_{2}$, corresponding to another $\mathrm{SU}(2)$ gauge group in F-theory. It is localized along the codimension one locus in $B_{n}$ given by

$$
t:=s_{3} b_{1}^{2}-s_{6} a_{1} b_{1}+s_{8} a_{1}^{2}=0,
$$

which is simply $f_{u}\left(0,-b_{1}, a_{1}\right)=0$. Denoting the divisor $t=0$ by abuse of notation by $t$ we note that its divisor class is

$$
\mathrm{SU}(2): \quad[t]=\left[s_{8}\right]+2\left[a_{1}\right]=-K_{B}+\left[a_{1}\right]+\left[b_{1}\right],
$$

where we used (3.17). We emphasize, that the divisor $t$ has an ordinary double point singularity at $a_{1}=b_{1}=0$ that can give rise, as we will see below, to symmetric matter representations in an F-theory model.

Thus, in summary we have found the following F-theory gauge group for the tuned model where we have unHiggsed the first U(1) associated to $\hat{s}_{Q}$ :

$$
G=\mathrm{SU}(2) \times \mathrm{SU}(2) \times \mathrm{U}(1) .
$$

We note that the $\mathrm{U}(1)$ associated to the section $\hat{s}_{R}$ remains. Thus, we denote the tuned geometry (5.8) by $X_{n+1}^{(1)}$, indicating the rank of its remaining MW-group.

The complete unHiggsing of the theory is achieved by tuning the complex structure of $X_{n+1}^{(1)}$ further so that the rational point $R$ is placed on top of the points $P=Q$ in the fiber $\mathcal{E}$. To this end, we have to impose according to (3.6)

$$
\Delta_{13}=a_{1} b_{3}-a_{3} b_{1} \equiv 0,
$$

where we again used the definition (4.1). Assuming again that we can apply procedure (A), where $\left[a_{3}\right]-\left[a_{1}\right]$ is effective, we take

$$
a_{3} v+b_{3} w \stackrel{!}{=} \lambda_{2}\left(a_{1} v+b_{1} w\right),
$$

so that (3.4) reduces to

$$
p=u f_{u}(u, v, w)+\lambda_{1} \lambda_{2}\left(a_{1} v+b_{1} w\right)^{3} .
$$


As before, we denote the divisor $\lambda_{2}=0$ simply by $\lambda_{2}$. The line bundles associated to the sections $\lambda_{1}$, cf. (5.3), and $\lambda_{2}$ in summary read

$$
\lambda_{1} \in \mathcal{O}\left(\left[a_{2}\right]-\left[a_{1}\right]\right) \quad \lambda_{2} \in \mathcal{O}\left(\left[a_{3}\right]-\left[a_{1}\right]\right) .
$$

The tuned model has trivial MW-group and is denoted by $X_{n+1}^{(0)}$. Its elliptic fibration has Kodaira fibers of type $I_{2}$ of the form $u f_{u}=0$ at the two codimension one loci $\lambda_{1}=0$ and $\lambda_{2}=0$ in $B_{n}$. In addition, the $I_{2}$-singularity of (5.8) on the divisor $t=0$ in (5.11) is enhanced to an $I_{3}$ singularity since (5.15) has an $A_{2}$-singularity at $u=f_{u}=a_{1} v+b_{1} w=0$. We will show later in section 5.7 that this is an $I_{3}^{\mathrm{S}}$-singularity, yielding an $\mathrm{SU}(3)$ gauge group in F-theory. We note that the presence of the ordinary double point singularity on $t$ will be crucial. In summary, the divisors of all three codimension one singularities are

$$
\begin{aligned}
& \mathrm{SU}(2):\left[\lambda_{1}\right]=\left[a_{2}\right]-\left[a_{1}\right], \\
& \mathrm{SU}(2):\left[\lambda_{2}\right]=\left[a_{3}\right]-\left[a_{1}\right], \\
& \mathrm{SU}(3):[t]=\left[s_{8}\right]+2\left[a_{1}\right] .
\end{aligned}
$$

In summary, the total gauge group of the unHiggsed theory is

$$
G_{\text {uni }}=\mathrm{SU}(2) \times \mathrm{SU}(2) \times \mathrm{SU}(3) .
$$

We see that, indeed, all abelian factors have been enhanced to a non-Abelian gauge symmetry. We emphasize that this is the generic maximal gauge group that can be present after imposing the tunings (5.2) and (5.13), modulo the possibility that one or more of the divisors $\lambda_{1}, \lambda_{2}, t$ may be reducible as mentioned at the end of section 5.2. For a particular gauge group factor in $G_{\text {uni }}$ to be actually present in a given model requires that the corresponding divisor class in (5.17) is non-trivial. Clearly, this depends on the choices of divisor classes $\left[a_{i}\right], i=1,2,3$, and $\left[s_{8}\right]$ in (3.17) entering the definition of the CY-manifold $X_{n+1}$. Furthermore, the particular embedding of the two U(1)-factors of F-theory on $X_{n+1}$ into $G_{\text {uni }}$ depends on this choice as well. We will analyze the different cases in the following section, starting with the simplest cases where the two U(1)'s are embedded as the Cartan generators of $\mathrm{SU}(2) \times \mathrm{SU}(2)$ or $\mathrm{SU}(3)$, before we focus on more complicated cases, where the U(1)'s can also be embedded into a linear combination of Cartan generators of different group factors in $G_{\text {uni }}$.

The specialized model. Before continuing with the discussion of the details of the unHiggsed model $X_{n+1}^{(0)}$, let us pause by analyzing the important specialized model (3.8) with $a_{1}=1$ and $b_{1}=0$ in some detail. The tunings (5.2) and (5.13) of the general model simplify in this case to

$$
b_{2}=b_{3}=0
$$

yielding the elliptic curve

$$
p=u f_{u}(u, v, w)+a_{2} a_{3} v^{3} .
$$

Clearly, this is identical to (5.15) with $\lambda_{1} \equiv a_{2}, \lambda_{2} \equiv a_{3}$ (and $a_{1}=1, b_{1}=0$ ) in the above discussion. Note that for any model $X_{n+1}$, there is a tuning of this type where we have 
fixed $b_{1}=0$ and performed the field redefinitions (5.7). The gauge group of the low energy effective theory is again $\mathrm{SU}(2) \times \mathrm{SU}(2) \times \mathrm{SU}(3)$ supported on the following divisors

$$
\mathrm{SU}(2):\left[a_{2}\right], \quad \mathrm{SU}(2):\left[a_{3}\right], \quad \mathrm{SU}(3):[t]=\left[s_{8}\right],
$$

where used that $t$ in (5.10) reduces to $s_{8}$ in the limit $a_{1}=1, b_{1}=0$.

\subsection{Weierstrass model from unHiggsing the general $\mathrm{U}(1)^{2}$ model}

We obtain the Weierstrass form of the Calabi-Yau manifold $X_{n+1}^{(0)}$ of the unHiggsed theory by inserting the tunings (5.4) and (5.14) into the general Weierstrass form of $X_{n+1}$ in appendix A. It reads

$$
\begin{aligned}
y^{2}=x^{3}+ & {\left[-\frac{1}{48}\left(s_{6}^{2}-4 s_{3} s_{8}\right)^{2}+\left(a_{1}\left(s_{2} s_{8}-\frac{1}{2} s_{5} s_{6}\right)+b_{1}\left(s_{3} s_{5}-\frac{1}{2} s_{2} s_{6}\right)\right) \lambda_{1} \lambda_{2} t\right] x z^{4} } \\
+ & {\left[\frac{1}{864}\left(s_{6}^{2}-4 s_{3} s_{8}\right)^{3}-\frac{1}{12}\left(s_{6}^{2}-4 s_{3} s_{8}\right)\left(a_{1}\left(s_{2} s_{8}-\frac{1}{2} s_{5} s_{6}\right)+b_{1}\left(s_{3} s_{5}-\frac{1}{2} s_{2} s_{6}\right)\right) \lambda_{1} \lambda_{2} t\right.} \\
& \left.+\left(\frac{1}{4}\left(b_{1} s_{2}-a_{1} s_{5}\right)^{2}-s_{1} t\right) \lambda_{1}^{2} \lambda_{2}^{2} t^{2}\right] z^{6}
\end{aligned}
$$

where we recall the definitions of the variables $\lambda_{1}, \lambda_{2}$ and $t$ in (5.8), (5.14) and (5.10), respectively.

We note that $f$ and $g$ do not vanish at any common codimension one locus, while the discriminant vanishes to order two and three at $\lambda_{1}=0, \lambda_{2}=0$ and $t=0$, respectively, indicating two $I_{2}$ and one $I_{3}$ singularities. This can be seen by computing $\Delta=4 f^{3}+27 g^{2}$ for the WSF (5.22) and reducing modulo (5.10),

$$
\Delta=\lambda_{1}^{2} \lambda_{2}^{2} t^{3} \Delta^{\prime}
$$

where $\Delta^{\prime}$ denotes the $I_{1}$ locus of the discriminant.

The Weierstrass model (5.22) matches precisely the general form (B.1) of a Weierstrass model with two $I_{2}$ singularities at $\lambda_{1}=0, \lambda_{2}=0$ and the leading Tate coefficients $\mathfrak{a}_{\mathfrak{i}}=$ $\left(\lambda_{1} \lambda_{2}\right)^{k} \mathfrak{a}_{i}^{(k)}$ of the form

$$
\begin{aligned}
& \mathfrak{a}_{1}^{(0)}=s_{6}, \quad \mathfrak{a}_{2}^{(0)}=-s_{3} s_{8}, \\
& \mathfrak{a}_{3}^{(1)}=\left(b_{1} s_{2}-a_{1} s_{5}\right) t, \quad \mathfrak{a}_{4}^{(1)}=\left(b_{1}\left(s_{3} s_{5}-s_{2} s_{6}\right)+a_{1} s_{2} s_{8}\right) t, \quad \mathfrak{a}_{6}^{(2)}=-s_{1} t^{3} .
\end{aligned}
$$

Thus, we confirm the presence of the gauge algebra $\mathrm{su}(2) \oplus \mathrm{su}(2)$.

Clearly, the Tate coefficients (5.24) do not have the correct orders of vanishing at $t=0$, namely $(0,1,1,2,3)$, required for an $I_{3}^{s}$ singularity in Tate's algorithm [58] In addition, the leading terms of the $t$-expansion of both $f$ and $g$ in the Weierstrass form (5.22) deviate from that of a standard $I_{3}^{\mathrm{s}}$ singularity at $t=0$ given in (B.11). This naively seems to imply that there is a monodromy acting on the fiber so that we have an $I_{3}^{\mathrm{ns}}$ singularity at $t=0$, corresponding to an $\mathrm{su}(2)$ gauge algebra. However, due to the singularity structure of the divisor $t=0$ and the subtle link to the form of $f, g$, the singularity is indeed an $I_{3}^{\mathrm{S}}$ corresponding to an $\mathrm{su}(3)$ gauge algebra. We will elaborate on this further in section 5.7. 
We just emphasize here that even for factoring out $t^{3}$ in $\Delta$ we had to exploit the special form of $t$ in terms of the sections $s_{i}, a_{1}$ and $b_{1}$ given in (5.10). In fact, if we had just used the general form (5.22) of the Weierstrass model for generic $t$ we would have obtained only an order two vanishing of $\Delta$ at $t=0$.

The specialized model. We obtain the Weierstrass form of the unHiggsed specialized model in (3.8) directly from the general Weierstrass model (5.22) by setting $a_{1}=1, b_{1}=0$. In this case, we have $\lambda_{1} \equiv a_{2}, \lambda_{2} \equiv a_{3}$ and $t \equiv s_{8}$, cf. (5.8), (5.14) and (5.10), yielding the Weierstrass form

$$
\begin{aligned}
y^{2}= & x^{3}+\left[-\frac{1}{48}\left(s_{6}^{2}-4 s_{3} t\right)^{2}+\left(s_{2} t-\frac{1}{2} s_{5} s_{6}\right) a_{2} a_{3} t\right] x z^{4} \\
& +\left[\frac{1}{864}\left(s_{6}^{2}-4 s_{3} t\right)^{3}-\frac{1}{12}\left(s_{6}^{2}-4 s_{3} t\right)\left(s_{2} t-\frac{1}{2} s_{5} s_{6}\right) a_{2} a_{3} t+\left(\frac{1}{4} s_{5}^{2}-s_{1} t\right) a_{2}^{2} a_{3}^{2} t^{2}\right] z^{6} .
\end{aligned}
$$

We emphasize that (5.25) is simultaneously of the general Weierstrass forms (B.19) with two $I_{2}$-singularities at $a_{2}=0$ and $a_{3}=0$ and one $I_{3}^{\mathrm{s}}$-singularity at $t=0$. The leading Tate coefficients $\mathfrak{a}_{i}=\mathfrak{a}_{i}^{(k, l)} t^{k}\left(\lambda_{1} \lambda_{2}\right)^{l}$ are

$$
\mathfrak{a}_{1}^{(0,0)}=s_{6}, \quad \mathfrak{a}_{2}^{(1,0)}=-s_{3}, \quad \mathfrak{a}_{3}^{(1,1)}=-s_{5}, \quad \mathfrak{a}_{4}^{(2,1)}=s_{2}, \quad \mathfrak{a}_{6}^{(3,2)}=-s_{1},
$$

This confirms the presence of an $\mathrm{su}(2) \oplus \mathrm{su}(2) \oplus \mathrm{su}(3)$ gauge algebra as claimed in (5.18). This also ensures that the unHiggsed model $X_{n+1}^{(0)}$ obtained by tuning the complex structure of $X_{n+1}$ reproduces all models with $\mathrm{su}(2) \oplus \mathrm{su}(2) \oplus \mathrm{su}(3)$ gauge algebra where the gauge factors are tuned on smooth divisors, including the special cases where only an su(2) $\oplus \mathrm{su}(2)$ or su(3) gauge algebra are realized, as we will demonstrate in more detail in sections 6.1, 6.2 and 6.3 .

\subsection{The matter spectrum}

We proceed with the determination of the charged matter spectrum of F-theory on the Calabi-Yau manifold $X_{n+1}^{(0)}$. The following analysis is based on the Weierstrass models (5.22) for the general and (5.25) for the specialized model.

Clearly, we have bi-fundamental matter at the mutual intersections of $\lambda_{1}, \lambda_{2}$ and $t$. The respective multiplicities are given as the product of the degrees of the two sections that vanish at the relevant intersection loci. In addition, there is fundamental matter under each factor of the gauge group in (5.18). The multiplicity of the fundamental matter of the two SU(2)'s is computed analogous to the multiplicities in (B.9), taking into account that the class of $\Delta^{\prime}$ in $(5.23)$ is changed to

$$
\left[\Delta^{\prime}\right]=-12 K_{B}-2\left[\lambda_{1}\right]-2\left[\lambda_{2}\right]-3[t]
$$

which follows as $[\Delta]=-12 K_{B}$. In addition, we have to subtract the order two loci $4 s_{3} s_{8}-s_{6}^{2}=\lambda_{1}=0$ and $4 s_{3} s_{8}-s_{6}^{2}=\lambda_{2}=0$, respectively, that support singularities of type $I I I$ which do not yield additional matter representations. 
The calculation of the multiplicity of the representation 3 of the the $\mathrm{SU}(3)$ is performed as follows. We find that $t=\Delta^{\prime}=0$ has two minimal associated prime ideals, one of which given by

$$
\mathfrak{p}=\left\{s_{6} b_{1}-2 s_{8} a_{1}, 2 s_{3} b_{1}-s_{6} a_{1}, s_{6}^{2}-4 s_{3} s_{1}\right\} .
$$

and the second one given by a large ideal $\mathfrak{J}$ with seven generators. Along the variety $V(\mathfrak{J})$, neither $f$ and $g$ vanish while $\Delta$ vanishes to order four, indicating an $I_{4}$-singularity, which signals the presence of fundamental matter. On $V(\mathfrak{p})$, the sections $f$ and $g$ both vanish to order two, while $\Delta$ vanishes to order four, corresponding to a Kodaira fibre of type $I V$ not giving rise to matter fields in F-theory. In addition, we check using the resultant technique of [9] that $V(\mathfrak{p})$ is of multiplicity three inside $\Delta^{\prime}$. This allows us to compute the class $[V(\mathfrak{J})]$, i.e. the contribution $x_{(\mathbf{1 , 1}, \mathbf{3})}^{\prime}$ of $I_{4}$-singularities to the number of matter fields in the representation $\mathbf{3}$. We obtain the multiplicity

$$
x_{(\mathbf{1}, \mathbf{1}, \mathbf{3})}^{\prime}=\left[\Delta^{\prime}\right]-3[V(\mathfrak{p})]=[t] \cdot\left(-9 K_{B}-2\left[\lambda_{1}\right]-2\left[\lambda_{2}\right]-3[t]\right),
$$

where we employed

$$
[V(\mathfrak{p})]=\left(\left[a_{1}\right]-K_{B}\right) \cdot\left(\left[s_{8}\right]+\left[a_{1}\right]\right)-\left[a_{1}\right] \cdot\left[b_{1}\right]=-K_{B} \cdot[t],
$$

using (3.17) and the class of $t$ in (5.11). Here, the first equality is obtained by multiplying the degrees of the first two generators of $\mathfrak{p}$, yielding the first summand, and subtracting the solution $a_{1}=b_{1}=0$ which does not satisfy the third generator of $\mathfrak{p}$.

In addition, we obtain adjoints for each gauge group. In 6D these are given by the topological genus of the curve supporting the respective gauge group factor. For the two $\mathrm{SU}(2)$ 's, it agrees with the arithmetic genus (B.4) of the generically smooth curves $\lambda_{1}=0$ and $\lambda_{2}=0$. There is one subtlety regarding the divisor $t=0$. Its arithmetic genus is the sum of the topological genus and the number $\left[a_{1}\right] \cdot\left[b_{1}\right]$ of double points. The matter supported at these double points $p_{g}$ can be either in the adjoint or symmetric plus antisymmetric representations, depending on the global properties of the resolution [42]. As we will argue in section 5.7, in the case at hand we have symmetrics plus anti-symmetrics. Thus, we have a number of $\left[a_{1}\right] \cdot\left[b_{1}\right]$ additional matter fields in the representations $(\mathbf{1}, \mathbf{1}, \mathbf{3})+$ $(\mathbf{1}, \mathbf{1}, \mathbf{6})$. Accordingly, the number of adjoints $x_{(\mathbf{1}, \mathbf{1}, \mathbf{8})}$ of the $\mathrm{SU}(3)$ in $6 \mathrm{D}$ is given by

$$
x_{(\mathbf{1}, \mathbf{1}, \mathbf{8})}=p_{g}=\frac{1}{2}[t] \cdot\left([t]+K_{B}\right)+1-\left[a_{1}\right] \cdot\left[b_{1}\right] .
$$

We summarize the full spectrum of the unHiggsed model (5.15) in table 4 where we used the divisor classes of $t, \lambda_{1}$ and $\lambda_{2}$ given in (5.17) and denote matter multiplicities in a representation $\mathbf{R}$ by $x_{\mathbf{R}}$.

Next, we readily check that all $6 \mathrm{D}$ anomalies are canceled for the spectrum in table 4 , see e.g. [59] for a review of $6 \mathrm{D}$ anomalies from an F-theory perspective. This is a crosscheck of the geometrical analysis of the unHiggsed model $X_{(n+1)}^{(0)}$.

Finally, we observe that the matter multiplicities in table 4 of the unHiggsed theory are related to those in table 2 of the abelian theory. We readily obtain the following relations between the multiplicities of the non-Abelian and the abelian theories: 


\begin{tabular}{|c|c|}
\hline Representation & Multiplicity \\
\hline$(\mathbf{1}, \mathbf{1}, \mathbf{6})$ & $x_{(\mathbf{1}, \mathbf{1}, \mathbf{6})}=\left[a_{1}\right] \cdot\left([t]+K_{B}-\left[a_{1}\right]\right)$ \\
\hline$(\mathbf{2}, \mathbf{2}, \mathbf{1})$ & $x_{(\mathbf{2}, \mathbf{2}, \mathbf{1})}=\left[\lambda_{1}\right] \cdot\left[\lambda_{2}\right]$ \\
\hline$(\mathbf{2}, \mathbf{1}, \mathbf{3})$ & $x_{(\mathbf{2}, \mathbf{1}, \mathbf{3})}=\left[\lambda_{1}\right] \cdot[t]$ \\
\hline$(\mathbf{1}, \mathbf{2}, \mathbf{3})$ & $x_{(\mathbf{1}, \mathbf{2}, \mathbf{3})}=\left[\lambda_{2}\right] \cdot[t]$ \\
\hline$(\mathbf{2}, \mathbf{1}, \mathbf{1})$ & $x_{(\mathbf{2}, \mathbf{1}, \mathbf{1})}=\left[\lambda_{1}\right] \cdot\left(-8 K_{B}-2\left[\lambda_{1}\right]-2\left[\lambda_{2}\right]-3[t]\right)$ \\
\hline$(\mathbf{1}, \mathbf{2}, \mathbf{1})$ & $x_{(\mathbf{1}, \mathbf{2}, \mathbf{1})}=\left[\lambda_{2}\right] \cdot\left(-8 K_{B}-2\left[\lambda_{1}\right]-2\left[\lambda_{2}\right]-3[t]\right)$ \\
\hline$(\mathbf{1}, \mathbf{1}, \mathbf{3})$ & $x_{(\mathbf{1}, \mathbf{1}, \mathbf{3})}=[t] \cdot\left(-9 K_{B}-2\left[\lambda_{1}\right]-2\left[\lambda_{2}\right]-3[t]\right)+x_{(\mathbf{1}, \mathbf{1}, \mathbf{6})}$ \\
\hline$(\mathbf{3}, \mathbf{1}, \mathbf{1})$ & $x_{(\mathbf{3}, \mathbf{1}, \mathbf{1})}=\frac{1}{2}\left[\lambda_{1}\right] \cdot\left(\left[\lambda_{1}\right]+K_{B}\right)+1$ \\
\hline$(\mathbf{1}, \mathbf{3}, \mathbf{1})$ & $x_{(\mathbf{1 , 3}, \mathbf{1})}=\frac{1}{2}\left[\lambda_{2}\right] \cdot\left(\left[\lambda_{2}\right]+K_{B}\right)+1$ \\
\hline$(\mathbf{1}, \mathbf{1}, \mathbf{8})$ & $x_{(\mathbf{1}, \mathbf{1}, \mathbf{8})}=\frac{1}{2}[t] \cdot\left([t]+K_{B}\right)+1-x_{(\mathbf{1}, \mathbf{1}, \mathbf{6})}$ \\
\hline
\end{tabular}

Table 4. Matter spectrum of the unHiggsed model $X_{n+1}^{(0)}$. The multiplicity of adjoints in $6 \mathrm{D}$ is included for completeness.

$$
\begin{gathered}
x_{(-2,-2)}=x_{(\mathbf{1}, \mathbf{1}, \mathbf{6})}, \\
x_{(0,2)}=x_{(\mathbf{2}, \mathbf{1}, \mathbf{3})}+2 x_{(\mathbf{3}, \mathbf{1}, \mathbf{1})}+x_{(\mathbf{1}, \mathbf{1}, \mathbf{6})}-2, \\
x_{(-1,-2)}=x_{(\mathbf{1}, \mathbf{2}, \mathbf{3})}+2 x_{(\mathbf{1}, \mathbf{3}, \mathbf{1}) \mathbf{8})}+x_{(\mathbf{1}, \mathbf{1}, \mathbf{6})}-2, \quad x_{(-2,-1)}=x_{(\mathbf{2}, \mathbf{1}, \mathbf{3})}+2 x_{(\mathbf{1}, \mathbf{1}, \mathbf{8})}-2, \\
x_{(-1,1)}=2 x_{(\mathbf{2}, \mathbf{2}, \mathbf{1})}+x_{(\mathbf{2}, \mathbf{1}, \mathbf{3})}+x_{(\mathbf{1}, \mathbf{2}, \mathbf{3})}+2 x_{(\mathbf{1}, \mathbf{1}, \mathbf{8})}-2, \\
x_{(1,0)}=x_{(\mathbf{1}, \mathbf{2}, \mathbf{3})}+2 x_{(\mathbf{2}, \mathbf{1}, \mathbf{1})}+x_{(\mathbf{1}, \mathbf{1}, \mathbf{3})}+x_{(\mathbf{1}, \mathbf{1}, \mathbf{6})}, \\
x_{(0,1)}=x_{(\mathbf{2}, \mathbf{1}, \mathbf{3})}+2 x_{(\mathbf{1}, \mathbf{2}, \mathbf{1})}+x_{(\mathbf{1}, \mathbf{1}, \mathbf{3})}+x_{(\mathbf{1}, \mathbf{1}, \mathbf{6})}, \\
x_{(1,1)}=2 x_{(\mathbf{2}, \mathbf{2}, \mathbf{1})}+x_{(\mathbf{2}, \mathbf{1}, \mathbf{3})}+x_{(\mathbf{1}, \mathbf{2}, \mathbf{3})}+x_{(\mathbf{1}, \mathbf{1}, \mathbf{3})}+x_{(\mathbf{1}, \mathbf{1}, \mathbf{6})} .
\end{gathered}
$$

Here we used (5.17) to express the divisor classes of $\lambda_{1}, \lambda_{2}$ and $t$ in terms of the classes $\left[a_{i}\right]$ with $i=1,2,3$ and $\left[s_{8}\right]$. These relations have a clear interpretation if we Higgs the nonAbelian theory back to the abelian one defined by $X_{n+1}$. Indeed, we can view these relations in terms of the Higgsing by at least two bi-fundamentals $(\mathbf{1}, \mathbf{2}, \mathbf{3})$ and $(\mathbf{2}, \mathbf{1}, \mathbf{3})$ each as discussed in section 6 . Then we employ the branchings of representations in (2.7), (2.8), (2.9) and (2.10) to obtain the spectrum the relations (5.32). Note that we have to subtract 2 as two states with charges $(2,1),(1,2)$ and $(-1,1)$, respectively, inside the two bifundamentals are eaten up by the massive gauge bosons. Alternatively, we can view the relations (5.32) as encoding the combination of Higgsings on adjoints of $\mathrm{SU}(2) \times \mathrm{SU}(2)$ and at least two fundamentals of an unbroken $\mathrm{SU}(3)$ or adjoints of $\mathrm{SU}(3)$ and at least two fundamentals of the unbroken $\mathrm{SU}(2) \times \mathrm{SU}(2)$. Both point of views will be crucial below in section 6 .

The specialized model. The matter spectrum of the specialized Calabi-Yau manifold after unHiggsing as given in (5.20) follows directly from that of the general model in table 4 by setting $\left[a_{1}\right]=0$. This yields the spectrum in table 5 , where we note that $\left[\lambda_{1}\right]=\left[a_{2}\right]$, $\left[\lambda_{2}\right]=\left[a_{3}\right]$ and $[t]=\left[s_{8}\right]$ according to (5.17). 


\begin{tabular}{|c|c|}
\hline Representation & Multiplicity \\
\hline$(\mathbf{2}, \mathbf{2}, \mathbf{1})$ & $x_{(\mathbf{2}, \mathbf{2}, \mathbf{1})}=\left[a_{2}\right] \cdot\left[a_{3}\right]$ \\
\hline$(\mathbf{2}, \mathbf{1}, \mathbf{3})$ & $x_{(\mathbf{2}, \mathbf{1}, \mathbf{3})}=\left[a_{2}\right] \cdot\left[s_{8}\right]$ \\
\hline$(\mathbf{1}, \mathbf{2}, \mathbf{3})$ & $x_{(\mathbf{1}, \mathbf{2}, \mathbf{3})}=\left[a_{3}\right] \cdot\left[s_{8}\right]$ \\
\hline$(\mathbf{2}, \mathbf{1}, \mathbf{1})$ & $x_{(\mathbf{2}, \mathbf{1}, \mathbf{1})}=\left[a_{2}\right] \cdot\left(-8 K_{B}-2\left[a_{2}\right]-2\left[a_{3}\right]-3\left[s_{8}\right]\right)$ \\
\hline$(\mathbf{1}, \mathbf{2}, \mathbf{1})$ & $x_{(\mathbf{1}, \mathbf{2}, \mathbf{1})}=\left[a_{3}\right] \cdot\left(-8 K_{B}-2\left[a_{2}\right]-2\left[a_{3}\right]-3\left[s_{8}\right]\right)$ \\
\hline$(\mathbf{1}, \mathbf{1}, \mathbf{3})$ & $x_{(\mathbf{1}, \mathbf{1}, \mathbf{3})}=\left[s_{8}\right] \cdot\left(-9 K_{B}-2\left[a_{2}\right]-2\left[a_{3}\right]-3\left[s_{8}\right]\right)$ \\
\hline$(\mathbf{3}, \mathbf{1}, \mathbf{1})$ & $x_{(\mathbf{3}, \mathbf{1}, \mathbf{1})}=\frac{1}{2}\left[a_{2}\right] \cdot\left(\left[a_{2}\right]+K_{B}\right)+1$ \\
\hline$(\mathbf{1}, \mathbf{3}, \mathbf{1})$ & $x_{(\mathbf{1}, \mathbf{3}, \mathbf{1})}=\frac{1}{2}\left[a_{3}\right] \cdot\left(\left[a_{3}\right]+K_{B}\right)+1$ \\
\hline$(\mathbf{1}, \mathbf{1}, \mathbf{8})$ & $x_{(\mathbf{1}, \mathbf{1}, \mathbf{8})}=\frac{1}{2}\left[s_{8}\right] \cdot\left(\left[s_{8}\right]+K_{B}\right)+1$ \\
\hline
\end{tabular}

Table 5. Matter spectrum of the unHiggsed specialized model $X_{n+1}^{(0)}$. The multiplicity of adjoints in $6 \mathrm{D}$ is included for completeness.

We emphasize that this agrees precisely with the spectrum of a general Weierstrass model with two $I_{2}$ and one $I_{3}$ singularity found in appendix B.3. This is not surprising since we already observed in (5.26) that the unHiggsed specialized model reproduces precisely the most general Weierstrass model (B.19) with these singularities.

Comparing with the spectrum in table 4 , we note that the main difference of the spectrum in table 5 is the absence of the symmetric representation $(\mathbf{1}, \mathbf{1}, \mathbf{6})$ of the $\mathrm{SU}(3)$. This is due to the fact that the divisor $s_{8}=0$ supporting the $\mathrm{SU}(3)$ gauge theory is smooth. Furthermore, the range of allowed values for $\left[a_{i}\right], i=2,3$, and $\left[s_{8}\right]$ is larger in the specialized compared to the general model, due to different effectiveness constraints. This yields different matter multiplicities in the unHiggsed specialized model.

We conclude by noting that the relations (5.32) also hold for the specialized models, i.e. we can use table 5 to precisely reproduce the spectrum of the specialized abelian model in table 3. As before these relations encode the possibility of Higgsing the non-Abelian model in different ways back to the abelian model.

\subsection{Special unHiggsings: $b_{i} \rightarrow 0$}

We have discussed in section 5.2 the possibility of situations where the only possible solution to $\Delta_{12}=\Delta_{13}=0$ is given by $b_{i} \rightarrow 0$, for $i=1,2,3$. As further explained at the beginning of section 5.3, this can be viewed as a specialized model (3.8) with one reducible coefficient $\tilde{a}_{2}=a_{1} a_{2}$ as in (5.7). This implies that this special unHiggsing, its matter spectrum and Higgsings back to the Abelian theory $X_{n+1}$ is already covered by the discussion in section 5.5. However it is instructive to spell out some details of this analysis.

We begin by noting that the fully unHiggsed model $X_{n+1}^{(0)}$ with $b_{i} \rightarrow 0$ is described by

$$
u f_{u}+a_{1} a_{2} a_{3} v^{3}=0,
$$

which has gauge group $\mathrm{SU}(2)^{3} \times \mathrm{SU}(3)$, as already seen above. Its Weierstrass form is given by (B.19), after setting $a_{1}=1, b_{1}=0$ and $t=s_{8}$ and then identifying $\lambda_{1}=a_{2} a_{1}, \lambda_{3}=a_{3}$. This confirms the presence of three $I_{2}$ and one $I_{3}^{\mathrm{s}}$ singularities. 


\begin{tabular}{|c|c|}
\hline Representation & Multiplicity \\
\hline$(\mathbf{2}, \mathbf{2}, \mathbf{1}, \mathbf{1})$ & $x_{(\mathbf{2}, \mathbf{2}, \mathbf{1}, \mathbf{1})}=\left[a_{1}\right] \cdot\left[a_{2}\right]$ \\
\hline$(\mathbf{2}, \mathbf{1}, \mathbf{2}, \mathbf{1})$ & $x_{(\mathbf{2}, \mathbf{1}, \mathbf{2}, \mathbf{1})}=\left[a_{1}\right] \cdot\left[a_{3}\right]$ \\
\hline$(\mathbf{1}, \mathbf{2}, \mathbf{2}, \mathbf{1})$ & $x_{(\mathbf{1}, \mathbf{2}, \mathbf{2}, \mathbf{1})}=\left[a_{2}\right] \cdot\left[a_{3}\right]$ \\
\hline$(\mathbf{2}, \mathbf{1}, \mathbf{1}, \mathbf{3})$ & $x_{(\mathbf{2}, \mathbf{1}, \mathbf{1}, \mathbf{3})}=\left[a_{1}\right] \cdot\left[s_{8}\right]$ \\
\hline$(\mathbf{1}, \mathbf{2}, \mathbf{1}, \mathbf{3})$ & $x_{(\mathbf{1}, \mathbf{2}, \mathbf{1}, \mathbf{3})}=\left[a_{2}\right] \cdot\left[s_{8}\right]$ \\
\hline$(\mathbf{1}, \mathbf{1}, \mathbf{2}, \mathbf{3})$ & $x_{(\mathbf{1}, \mathbf{1}, \mathbf{2}, \mathbf{3})}=\left[a_{3}\right] \cdot\left[s_{8}\right]$ \\
\hline$(\mathbf{2}, \mathbf{1}, \mathbf{1}, \mathbf{1})$ & $x_{(\mathbf{2}, \mathbf{1}, \mathbf{1}, \mathbf{1})}=\left[a_{1}\right] \cdot\left(-8 K_{B}-2\left(\left[a_{1}\right]+\left[a_{2}\right]+\left[a_{3}\right]\right)-3\left[s_{8}\right]\right)$ \\
\hline$(\mathbf{1}, \mathbf{2}, \mathbf{1}, \mathbf{1})$ & $x_{(\mathbf{1}, \mathbf{2}, \mathbf{1}, \mathbf{1})}=\left[a_{2}\right] \cdot\left(-8 K_{B}-2\left(\left[a_{1}\right]+\left[a_{2}\right]+\left[a_{3}\right]\right)-3\left[s_{8}\right]\right)$ \\
\hline$(\mathbf{1}, \mathbf{1}, \mathbf{2}, \mathbf{1})$ & $x_{(\mathbf{1}, \mathbf{1}, \mathbf{2}, \mathbf{1})}=\left[a_{3}\right] \cdot\left(-8 K_{B}-2\left(\left[a_{1}\right]+\left[a_{2}\right]+\left[a_{3}\right]\right)-3\left[s_{8}\right]\right)$ \\
\hline$(\mathbf{1}, \mathbf{1}, \mathbf{1}, \mathbf{3})$ & $x_{(\mathbf{1}, \mathbf{1}, \mathbf{1}, \mathbf{3})}=\left[s_{8}\right] \cdot\left(-9 K_{B}-2\left(\left[a_{1}\right]+\left[a_{2}\right]+\left[a_{3}\right]\right)-3\left[s_{8}\right]\right)$ \\
\hline$(\mathbf{3}, \mathbf{1}, \mathbf{1}, \mathbf{1})$ & $x_{(\mathbf{3}, \mathbf{1}, \mathbf{1}, \mathbf{1})}=\frac{1}{2}\left[a_{1}\right] \cdot\left(\left[a_{1}\right]+K_{B}\right)+1$ \\
\hline$(\mathbf{1}, \mathbf{3}, \mathbf{1}, \mathbf{1})$ & $x_{(\mathbf{1}, \mathbf{3}, \mathbf{1}, \mathbf{1})}=\frac{1}{2}\left[a_{2}\right] \cdot\left(\left[a_{2}\right]+K_{B}\right)+1$ \\
\hline$(\mathbf{1}, \mathbf{1}, \mathbf{3}, \mathbf{1})$ & $x_{(\mathbf{1}, \mathbf{1}, \mathbf{3}, \mathbf{1})}=\frac{1}{2}\left[a_{3}\right] \cdot\left(\left[a_{3}\right]+K_{B}\right)+1$ \\
\hline$(\mathbf{1}, \mathbf{1}, \mathbf{1}, \mathbf{8})$ & $x_{(\mathbf{1}, \mathbf{1}, \mathbf{1}, \mathbf{8})}=\frac{1}{2}\left[s_{8}\right] \cdot\left(\left[s_{8}\right]+K_{B}\right)+1$ \\
\hline
\end{tabular}

Table 6. Matter spectrum of the specialized unHiggsins $b_{i} \rightarrow 0, i=1,2,3$. The multiplicity of adjoints in $6 \mathrm{D}$ is included for completeness.

The matter spectrum of the theory can be computed from analysis of this Weierstrass form (or 6D anomaly cancellation). It is given in table 6, which is completely symmetric under an exchange of SU(2)-factors. The multipliticies of bifundamentals and adjoints follow as before. For the multiplicities of fundamentals, we account for the third $\mathrm{SU}(2)$ by modifying the fourth to sixth lines in table 5 as follows. We just have to take into account in the parenthesis that we have a reducible $a_{2}$, i.e. $a_{2} \rightarrow a_{1} a_{2}$ so that $-2\left[a_{2}\right] \rightarrow-2\left(\left[a_{1}\right]+\left[a_{2}\right]\right)$.

The Higgsing to the Abelian model goes in steps. Basically the Higgses are certain components in all three possible bifundamental representations of one $\mathrm{SU}(2)$ and the $\mathrm{SU}(3)$. We obtain

$$
\begin{gathered}
\mathrm{SU}(2)_{a_{1}} \times \mathrm{SU}(2)_{a_{2}} \times \mathrm{SU}(2)_{a_{3}} \times \mathrm{SU}(3)_{s_{8}} \longrightarrow \mathrm{SU}(2)_{a_{1}} \times \mathrm{SU}(2)_{a_{2}} \times \mathrm{U}(1)^{\prime} \times \mathrm{SU}(2)_{s_{8}} \\
\longrightarrow \mathrm{SU}(2)_{a_{1}} \times \mathrm{U}(1)^{\prime} \times \mathrm{U}(1)^{\prime \prime} \rightarrow \mathrm{U}(1) \times \mathrm{U}(1)
\end{gathered}
$$

where we have indicated the codimension one loci of the various gauge group factors by a subscript. Note also that the U(1)'s after the second and third Higgsing are different. The first Higgsing is performed by the state with Dynkin labels $(0 ; 0 ; 1 ; 1,0)$ inside $(\mathbf{1}, \mathbf{1}, \mathbf{2}, \mathbf{3})$, the second Higgsing by the state $(0 ;-1 ; 0 ; 0,-1)$ inside the representation $(\mathbf{1}, \mathbf{2}, \mathbf{2})_{(0)}$ of the original $(\mathbf{1}, \mathbf{2}, \mathbf{1}, \mathbf{3})$, and the final Higgsing by the state $(1 ; 0 ; 0 ;-1,1)$ inside the $\mathbf{2}_{(-1,1)}$ of the original $(\mathbf{2}, \mathbf{1}, \mathbf{1}, \mathbf{3})$. Then the final unbroken $\mathrm{U}(1)$ generators are

$$
\xi=\sigma_{3}^{(1)}-\sigma_{3}^{(3)}+\lambda, \quad \zeta=\sigma_{3}^{(1)}+\sigma_{3}^{(2)}-(\lambda-\mu),
$$


where $\sigma_{3}^{(i)}$ denotes the third Cartan matrix of the i-th $\mathrm{SU}(2)$ and $\lambda=\operatorname{diag}(1,-1,0)$, $\mu=\operatorname{diag}(1,0,-1) .{ }^{10}$ For these $\mathrm{U}(1)$ generators, we see that the states $(-1 ; 0 ; 0 ;-1,1)$ inside $(\mathbf{2}, \mathbf{1}, \mathbf{1}, \mathbf{3})$ and the states $\pm(2 ; 0 ; 0 ; 0,0)$ inside $(\mathbf{3}, \mathbf{1}, \mathbf{1}, \mathbf{1})$ will have charges $(-2,-2)$, i.e. the $(-2,-2)$ matter arises as some sort of "diagonal adjoint" and the first $\mathrm{SU}(2) \times$ $\mathrm{SU}(3)$ bifundamental. It does not appear as an ordinary double point singularity of the $\mathrm{SU}(3)$ divisor.

The multiplicities of the Higgsed Abelian theory $X_{(n+1)}$ in table 2 are related to those of the unHiggsed non-Abelian theory in table 6 by the following relations:

$$
\begin{aligned}
x_{(0,2)} & =x_{(\mathbf{1}, \mathbf{1}, \mathbf{2}, \mathbf{3})}+2 x_{(\mathbf{1}, \mathbf{1}, \mathbf{3}, \mathbf{1})}-2, \\
x_{(2,0)} & =x_{(\mathbf{1}, \mathbf{2}, \mathbf{1}, \mathbf{3})}+2 x_{(\mathbf{1}, \mathbf{3}, \mathbf{1}, \mathbf{1})}-2, \\
x_{(-2,-2)} & =x_{(\mathbf{2}, \mathbf{1}, \mathbf{1}, \mathbf{3})}+2 x_{(\mathbf{3}, \mathbf{1}, \mathbf{1}, \mathbf{1})}-2, \\
x_{(-2,-1)} & =x_{(\mathbf{2}, \mathbf{1}, \mathbf{1}, \mathbf{3})}+x_{(\mathbf{1}, \mathbf{2}, \mathbf{1}, \mathbf{3})}+2 x_{(\mathbf{1}, \mathbf{1}, \mathbf{1}, \mathbf{8})}+2 x_{(\mathbf{2}, \mathbf{2}, \mathbf{1}, \mathbf{1})}-2, \\
x_{(-1,-2)} & =x_{(\mathbf{2}, \mathbf{1}, \mathbf{1}, \mathbf{3})}+x_{(\mathbf{1}, \mathbf{1}, \mathbf{2}, \mathbf{3})}+2 x_{(\mathbf{1}, \mathbf{1}, \mathbf{1}, \mathbf{8})}+2 x_{(\mathbf{2}, \mathbf{1}, \mathbf{2}, \mathbf{1})}-2, \\
x_{(-1,1)} & =x_{(\mathbf{1}, \mathbf{2}, \mathbf{1}, \mathbf{3})}+x_{(\mathbf{1}, \mathbf{1}, \mathbf{2}, \mathbf{3})}+2 x_{(\mathbf{1}, \mathbf{1}, \mathbf{1}, \mathbf{8})}+2 x_{(\mathbf{1}, \mathbf{2}, \mathbf{2}, \mathbf{1})}-2, \\
x_{(0,1)} & =2 x_{(\mathbf{2}, \mathbf{2}, \mathbf{1}, \mathbf{1})}+x_{(\mathbf{2}, \mathbf{1}, \mathbf{1}, \mathbf{3})}+x_{(\mathbf{1}, \mathbf{2}, \mathbf{1}, \mathbf{3})}+2 x_{(\mathbf{1}, \mathbf{1}, \mathbf{2}, \mathbf{1})}+x_{(\mathbf{1}, \mathbf{1}, \mathbf{1}, \mathbf{3})}, \\
x_{(1,0)} & =2 x_{(\mathbf{2}, \mathbf{1}, \mathbf{2}, \mathbf{1})}+x_{(\mathbf{1}, \mathbf{1}, \mathbf{2}, \mathbf{3})}+x_{(\mathbf{2}, \mathbf{1}, \mathbf{1}, \mathbf{3})}+2 x_{(\mathbf{1}, \mathbf{2}, \mathbf{1}, \mathbf{1})}+x_{(\mathbf{1}, \mathbf{1}, \mathbf{1}, \mathbf{3})}, \\
x_{(1,1)} & =2 x_{(\mathbf{1}, \mathbf{2}, \mathbf{2}, \mathbf{1})}+x_{(\mathbf{1}, \mathbf{1}, \mathbf{2}, \mathbf{3})}+x_{(\mathbf{1}, \mathbf{2}, \mathbf{1}, \mathbf{3})}+2 x_{(\mathbf{2}, \mathbf{1}, \mathbf{1}, \mathbf{1})}+x_{(\mathbf{1}, \mathbf{1}, \mathbf{1}, \mathbf{3})} .
\end{aligned}
$$

This shows that the spectrum of the Abelian model $X_{n+1}$ will indeed fall into representations of $\mathrm{SU}(2)^{3} \times \mathrm{SU}(3)$ after unHiggsing.

\subsection{Novel Weierstrass models with $I_{3}^{\mathrm{s}}$ singularities}

We conclude the discussion of the tuned Calabi-Yau manifold $X_{n+1}^{(0)}$ by a detailed analysis of the global structure of its Weierstrass model and the interplay with the singularities of the divisor $t=0$ supporting the $I_{3}$-singularity.

The central object to distinguish between the split and non-split form of a Kodaira singularity of type $I_{3}$ is the monodromy cover [60]

$$
\Psi^{2}+\left.\frac{9 g}{2 f}\right|_{t=0}=0
$$

The standard story in F-theory is that we have a split $I_{3}$, corresponding to an su(3) gauge algebra, if $\frac{9 g}{2 f}$ evaluated at $t=0$ is a perfect square and otherwise a non-split $I_{3}$, which yields just an $\mathrm{sp}(1) \cong \mathrm{su}(2)$. This condition is determined locally from the structure of the Weierstrass model around $t=0$. While in most applications considered in the literature the monodromy can be determined in a straightforward fashion from the geometry using global considerations, we demonstrate here an example of the subtlety that may arise if $t=0$ is singular and we are not in a unique factorization domain. In the situation we consider here it turns out that $\frac{9 g}{2 f}$ is a square locally around $t=0$, though this is encoded in a nontrivial fashion in the algebra.

\footnotetext{
${ }^{10}$ Note that the Dynkin labels have been computed here w.r.t. $\lambda$ and $\lambda-\mu$, as usual.
} 

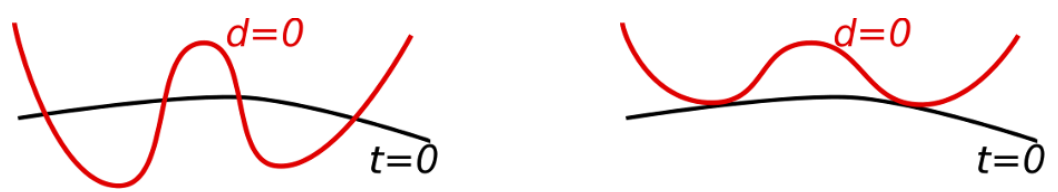

Figure 10. Generic (left) and non-generic (right) $I_{3}^{\mathrm{ns}}$ along the divisor $t=0$ with normal and tangential crossing of $t=0$ and $d=0$, respectively.

We begin by evaluating the monodromy cover (5.37) for the Weierstrass form (5.22). We obtain

$$
\left.\frac{9 g}{2 f}\right|_{t=0}=\frac{1}{4}\left(s_{6}^{2}-4 s_{3} s_{8}\right)=: \frac{1}{4} d .
$$

It is one key property of the Weierstrass form that this $d$ is at the same time the discriminant of the divisor $t=s_{8} a_{1}^{2}-s_{6} a_{1} b_{1}+s_{3} b_{1}^{2}=0$ viewed as a conic in the variables $a_{1}, b_{1}$. The consequences of this coincidence are most obvious by contrasting the geometry at hand with a generic situation. In general, the intersection points of $t=0$ and $\frac{9 g}{2 f}=0$ are the branch points around which, in a resolution, two nodes in the reducible fiber over $t=0$ are exchanged by a $\mathbb{Z}_{2}$-monodromy, cf. the left figure in figure in 10 . This reduces the gauge algebra from $\mathrm{su}(3)$ to $\mathrm{su}(2)$ [61]. In contrast, for the geometry at hand we have a non-generic divisor $t=0$ with double-points at $a_{1}=b_{1}=0$ and discriminant $d=0$, which agrees with (5.38). Thus, $t=0$ intersects $d$ tangentially, i.e. $t=d=0$ have only double zeros, see the right picture in figure 10. This double zero arises since two zeros of multiplicity one have merged. Furthermore, as the zeros of $d=0$ along $t=0$ are also the branch points of the monodromy cover we see that its branch points have come together in pairs. As the monodromy around a pair of branch points is trivial, no nodes in the resolution are not interchanged, leaving a full su(3) gauge algebra.

The fact that the divisors $d$ and $t$ intersect only at points of tangency can be seen in a simple fashion by considering the normal vector to each of the divisors at the intersection points. Where $t=d=0$ we have

$$
\left(s_{8} a_{1}^{2}+s_{3} b_{1}^{2}\right)^{2}=s_{6}^{2} a_{1}^{2} b_{1}^{2}=4 s_{3} s_{8} a_{1}^{2} b_{1}^{2},
$$

from which it follows that $s_{8} a_{1}^{2}=s_{3} b_{1}^{2}=s_{6} a_{1} b_{1} / 2$, and

$$
2 s_{8} a_{1}=s_{6} b_{1}, \quad 2 s_{3} b_{1}=s_{6} a_{1} .
$$

We can then relate the differentials of $d$ and $t$,

$$
\begin{aligned}
a_{1} b_{1} d(d) & =\left(a_{1} b_{1}\right)\left(2 s_{6} d s_{6}-4 s_{3} d s_{8}-4 s_{8} d s_{3}\right) \\
& =\left(-2 s_{6}\right) d(t)
\end{aligned}
$$

which shows that the normals are proportional and hence the intersection is at a point of tangency.

We emphasize that it is also this interplay between the structure of $f, g$ and the divisor $t=0$ that does not permit us to deform $t$ so that its ordinary double singularities disappear. In fact, if we changed the structure of $t$ without changing the Weierstrass form (5.22), we 
would only have an order two vanishing of $\Delta$ at $t=0$ and just an $I_{2}$-singularity with an $\mathrm{su}(2)$ gauge algebra, as noted above. If we changed only the Weierstrass form, e.g. by modifying the leading coefficient of $f$ and $g$, while keeping $t$ unchanged, we might get an $I_{3}^{\mathrm{ns}}$ and again only an $\mathrm{su}(2)$ gauge algebra. Thus, the ordinary double points of $t=0$ are able to support symmetric plus anti-symmetric matter representations, in such a way that the geometry cannot be deformed to a smooth discriminant locus which would support only adjoint representations [42]. It is an interesting open question whether symmetric matter representations can only be formed in this nontrivial algebraic fashion, or may also arise when smooth divisors are deformed into singular divisors with double point singularities.

Let us conclude by showing in another way that the zeros of $t=d=0$ are indeed double-points. To this end, we define the ideal

$$
\mathcal{I}:=\{t, d\}=\left\{s_{8} a_{1}^{2}-s_{6} a_{1} b_{1}+s_{3} b_{1}^{2}, s_{6}^{2}-4 s_{3} s_{1}\right\}
$$

Generically, there would be $\operatorname{deg}(t) \cdot \operatorname{deg}(d)=-2 K_{B} \cdot\left(\left[s_{8}\right]+2\left[a_{1}\right]\right)$ points of multiplicity one in the vanishing set $V(\mathcal{I})$. Computing the primary decomposition of $\mathcal{I}$ we obtain only one prime ideal $\mathfrak{p}$ that is given in (5.28), showing the irreducibility of $V(\mathcal{I})$. However, the corresponding variety $V(\mathfrak{p})$ has multiplicity two inside $V(\mathcal{I})$ as can be seen using the resultant technique [9]. In other words $V(\mathcal{I})$ consistent only of points of multiplicity two, i.e. double zeros. Their number is computed by the class

$$
[V(\mathfrak{p})]=-K_{B} \cdot\left(\left[s_{8}\right]+2\left[a_{1}\right]\right)=\frac{1}{2} \operatorname{deg}(t) \cdot \operatorname{deg}(d)
$$

according to (5.30), which is half the product of the degrees of $t$ and $d$ as expected.

Performing a local computation around an intersection point in $V(\mathfrak{p})$, we can show explicitly that the tangents of $t=0$ and $d=0$ become parallel when they intersect. This implies that locally around intersection points with $t=0$, the constraint $d$ becomes a perfect square to leading order, so that the monodromy cover (5.37) is reducible, corresponding to a Kodaira singularity of type $I_{3}^{\mathrm{s}}$.

\section{UnHiggsing of two U(1)'s: concrete examples}

In this section we systematically describe examples of the unHiggsing of two $\mathrm{U}(1)$ factors. Following the spirit of section 2, we discuss first the rank-preserving unHiggsings to $\mathrm{SU}(2)$ $\times \mathrm{SU}(2)$ and $\mathrm{SU}(3)$, and then those to a fully fledged $\mathrm{SU}(2) \times \mathrm{SU}(2) \times \mathrm{SU}(3)$ hybrid model. The discussion here is based on the unHiggsings of type A described in the previous section; in the completely general cases where approach A cannot be used directly, as discussed earlier we can unHiggs using approach $\mathrm{D}$, giving a model which is equivalent to one with unHiggsings of type A but with a reducible divisor $\tilde{a}_{2}$.

\subsection{UnHiggsing of two $\mathrm{U}(1)$ 's to $\mathrm{SU}(2) \times \mathrm{SU}(2)$}

We begin with the discussion of Abelian F-theory models that admit an unHiggsing to $\mathrm{SU}(2) \times \mathrm{SU}(2)$ with adjoints. The two $\mathrm{U}(1)$-factors are embedded as the Cartan generators 
of $\mathrm{SU}(2) \times \mathrm{SU}(2)$ in the adjoint Higgsing

$$
\mathrm{U}(1) \times \mathrm{U}(1) \stackrel{\text { adjoints }}{\stackrel{b_{2}, b_{3} \rightarrow 0}{\longrightarrow}} \mathrm{SU}(2) \times \mathrm{SU}(2) .
$$

As we will show below, these Higgsings occur only for certain Calabi-Yau manifolds $X_{n+1}$ with $b_{1}=0$ and $\left[a_{1}\right]=0$, i.e. cases that are included in the specialized model of section 3.1, if we tune $b_{2}, b_{3} \rightarrow 0$ as indicated. In addition, we have to have $\left[a_{2}\right],\left[a_{3}\right] \geq-K_{B}$, which ensures the presence of adjoints of the two $\mathrm{SU}(2)$ 's on these divisors, and $\left[s_{8}\right]=0$, which is required to guarantee the absence of the additional $\mathrm{SU}(3)$ gauge group.

\subsubsection{Geometric discussion}

To proceed more explicitly, we rewrite the effectiveness condition for the section $s_{1}$ in (3.17) in terms of the classes of $\lambda_{1}, \lambda_{2}$ and $t$ defined in (5.17) as

$$
-6 K_{B}-2\left[\lambda_{1}\right]-2\left[\lambda_{2}\right]-3[t] \geq 0 .
$$

From this it is clear that the two $\mathrm{SU}(2)$ 's can only both carry adjoints, which is the case if $\left[\lambda_{1}\right],\left[\lambda_{2}\right]$ are of the form $-K_{B}+Z$ for an effective class $Z$, if the class of $t$ is strictly smaller than $-K_{B}$. As $[t] \geq-K_{B}$ by (5.11) unless $b_{1} \equiv 0$ (the choice $a_{1} \equiv 0$ is equivalent under $v \leftrightarrow w)$, which also imposes $\left[a_{1}\right]=0$ by the assumption of a smooth Abelian model, ${ }^{11}$ we conclude that only the specialized models (3.8) can admit an unHiggsing to two SU(2)'s with adjoints. In this case we have $\lambda_{1} \equiv a_{2}, \lambda_{2} \equiv a_{3}$ and the Calabi-Yau manifold $X_{n+1}^{(0)}$ describing the unHiggsed theory is given by (5.20).

In order to have a clear unHiggsing to two $\mathrm{SU}(2)$ 's we have to require $\left[s_{8}\right]=0$ so that the $\mathrm{SU}(3)$ in $G_{\text {uni }}$ is absent. Then the two parameters $b_{2}$ and $b_{3}$ that are switched off in the tuning (5.19) triggering the unHiggsing obey the relation

$$
\left[b_{i}\right]=\left[a_{i}\right]+K_{B}, \quad i=2,3
$$

as follows from (3.19). Thus, the number of independent VEVs of the $1+\frac{1}{2}\left[a_{i}\right] \cdot\left(\left[a_{i}\right]+K_{B}\right)$ adjoint Higgses agrees precisely with the number of monomials in the respective $b_{i}$ that is tuned for the unHiggsing, as can be seen on a concrete base, e.g. $B_{n}=\mathbb{P}^{2}$.

Geometrically, the unHiggsing (6.1) corresponds to a transition of the generators of the Mordell-Weil group of $X_{n+1}$ corresponding to the U(1)'s into the Cartan divisors of the two $I_{2}$-singularities supporting the $\mathrm{SU}(2)$ gauge groups. In other words, a horizontal divisor given by a section of the elliptic fibration of $X_{n+1}$ is transformed into a vertical divisor in $X_{n+1}^{(0)}$ localized at a codimension one locus in the base $B_{n}$.

In the Weierstrass form, the unHiggsing $b_{2}, b_{3} \rightarrow 0$ of the Abelian theory produces the model (5.25). As pointed out earlier it precisely agrees with the general Weierstrass model (B.19) with two $I_{2^{-}}$and one $I_{3}^{\mathrm{s}}$-singularities. If we set $\left[s_{8}\right]=0$ the $I_{3}^{s}$-singularity is absent and we simply reproduce the most general model with two $I_{2}$-singularities, cf. (B.1), with the identification

$$
\sigma=a_{2}, \quad \tau=a_{3} .
$$

This direct matching of Weierstrass models with two $I_{2}$ singularities ensures that any such model can be obtained by unHiggsing our Abelian model defined via $X_{n+1}$.

\footnotetext{
${ }^{11}$ Recall the presence of an $I_{2}$ singularity at $a_{1}=0$ if $b_{1} \equiv 0$.
} 


\subsubsection{UnHiggsing from matching of spectra}

Next, we note that the identification of Abelian models that unHiggs to two SU(2)'s can performed directly from investigation of the spectrum of the general Abelian model.

Recalling the discussion of section 2.2.1, an Abelian model with two U(1)'s arising from a broken $\mathrm{SU}(2) \times \mathrm{SU}(2)$ has to have a charge lattice as shown in figure 1, i.e. the matter with charges $(-2,-2),(-2,-1)$ and $(-1,-2)$ has to be absent while matter with charges $(2,0)$ and $(0,2)$ has to be generically present. It follows directly from table 2 that the unique solution to this is precisely $\left[a_{1}\right]=\left[s_{8}\right]=0$, consistent with the above geometric arguments.

Furthermore, the spectrum of the Abelian model of table 2 then automatically assumes the form of an $\mathrm{SU}(2) \times \mathrm{SU}(2)$ gauge theory. Indeed, we find that the non-zero multiplicities of the Abelian theory obey the relations

$$
\begin{aligned}
x_{(2,0)} & =\left[a_{2}\right] \cdot\left(K_{B}+\left[a_{2}\right]\right)=2 x_{(\mathbf{3}, \mathbf{1})}-2, & & x_{(0,2)}=\left[a_{3}\right] \cdot\left(K_{B}+\left[a_{3}\right]\right)=2 x_{(\mathbf{1}, \mathbf{3})}-2, \\
x_{(1,0)} & =-4\left[a_{2}\right] \cdot\left(4 K_{B}+\left[a_{2}\right]+\left[a_{3}\right]\right)=2 x_{(\mathbf{2}, \mathbf{1})}, & & x_{(0,1)}=-4\left[a_{3}\right] \cdot\left(4 K_{B}+\left[a_{2}\right]+\left[a_{3}\right]\right)=2 x_{(\mathbf{1}, \mathbf{2})}, \\
x_{(-1,1)} & =x_{(1,1)}=2\left[a_{2}\right] \cdot\left[a_{3}\right]=2 x_{(\mathbf{2}, \mathbf{2})}, & &
\end{aligned}
$$

with the multiplicities $x_{\mathbf{R}}$ of matter in a representation $\mathbf{R}$ of the $\mathrm{SU}(2) \times \mathrm{SU}(2)$ gauge group. These relations ensure that the spectrum of the theory falls into representations of $\mathrm{SU}(2) \times \mathrm{SU}(2)$ in the limit $b_{2}, b_{3} \rightarrow 0$. In fact, (6.5) follows directly from the branching rules of $\mathrm{SU}(2) \times \mathrm{SU}(2)$ representations in an adjoint Higgsing as discussed in section 2.2.1 and a comparison of tables 5 and 3 . Note that we have to subtract 1 from the multiplicities $x_{(\mathbf{3}, \mathbf{1})}$, $x_{(\mathbf{1}, \mathbf{3})}$ of the adjoints as one field is eaten up by the massive gauge bosons, respectively.

We conclude by noting that the relations (6.5) formally follow also from the general relations (5.32). To this end, we have to set all multiplicities to zero that involve non-trivial representations of $\mathrm{SU}(3)$, which includes the -2 in $x_{(-1,1)}$.

\subsubsection{Examples: models on $\mathbb{P}^{2}$}

We conclude by demonstrating the general observations made in this section for a simple and concrete example. We consider a Calabi-Yau threefold $X_{3}$ with base $B_{2}=\mathbb{P}^{2}$. We solve the effectiveness constraints (3.19) and perform the tunings (5.19) of the complex structure of $X_{3}$ yielding $X_{3}^{(0)}$. We then obtain the following list of models with $\left[s_{8}\right]=0$ :

\begin{tabular}{|c|c||c|c|c|c|c|c|c|c|}
\hline$\left[a_{2}\right]$ & {$\left[a_{3}\right]$} & $b_{2}$ & $b_{3}$ & $s_{1}$ & $s_{2}$ & $s_{3}$ & $s_{5}$ & $s_{6}$ & $s_{8}$ \\
\hline 6 & 3 & 3 & 0 & 0 & 3 & 6 & 0 & 3 & 0 \\
5 & 4 & 2 & 1 & 0 & 3 & 6 & 0 & 3 & 0 \\
5 & 3 & 2 & 0 & 2 & 4 & 6 & 1 & 3 & 0 \\
4 & 5 & 1 & 2 & 0 & 3 & 6 & 0 & 3 & 0 \\
4 & 4 & 1 & 1 & 2 & 4 & 6 & 1 & 3 & 0 \\
4 & 3 & 1 & 0 & 4 & 5 & 6 & 2 & 3 & 0 \\
3 & 6 & 0 & 3 & 0 & 3 & 6 & 0 & 3 & 0 \\
3 & 5 & 0 & 2 & 2 & 4 & 6 & 1 & 3 & 0 \\
3 & 4 & 0 & 1 & 4 & 5 & 6 & 2 & 3 & 0 \\
3 & 3 & 0 & 0 & 0 & 6 & 6 & 3 & 3 & 0 \\
\hline
\end{tabular}


As expected, this precisely reproduces the list of models (B.7) based on the completely general WSF (B.1). In addition, we check the necessary relation between the degrees of the polynomials, $\left[a_{i}\right]-\left[b_{i}\right]=3, i=2,3$. This ensures that the number of adjoint VEVs agrees precisely with the monomials in $b_{2}, b_{3}$, that have been switched off in the unHiggsing procedure.

We emphasize again that the Abelian theory resulting from Higgsing $\mathrm{SU}(2) \times \mathrm{SU}(2)$ via adjoints can not be described by F-theory on the $d P_{2}$-elliptic fibrations in [8-12]. Thus, we see that the Calabi-Yau manifolds $X_{n+1}$ are crucial for describing the branch in the moduli space of F-theory with two Abelian factors.

\subsection{UnHiggsings of two $\mathrm{U}(1)$ 's to $\mathrm{SU}(3)$}

Next, we turn to F-theory compactifications with two U(1)'s that are embedded into the Cartan subalgebra of an SU(3) via the adjoint Higgsing

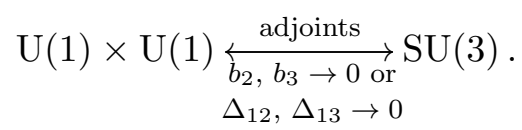

We will demonstrate that this is only possible for models with either $\left[a_{1}\right]=0$ and $\left[s_{8}\right] \geq$ $-K_{B}$ by tuning $b_{2}=b_{3} \equiv 0$ or with $\left[a_{1}\right],\left[b_{1}\right]>0$ (where $\left[s_{8}\right]$ might or might not obey $\left.\left[s_{8}\right] \geq-K_{B}\right)$ by tuning $\Delta_{12}=\Delta_{13} \equiv 0 .{ }^{12}$ These conditions guarantee the presence of adjoints of the $\mathrm{SU}(3)$. Additionally, we require $\left[a_{2}\right]=\left[a_{3}\right]=0$ in the first case and $\left[a_{1}\right]=\left[a_{2}\right]=\left[a_{3}\right]$ in the second case to ensure that no $\mathrm{SU}(2)$ 's are present. We emphasize that the latter models admit SU(3)-divisors with ordinary double point singularities, giving rise to symmetric matter representations.

\subsubsection{Geometric discussion}

We organize our discussion by analyzing the two types of models with unHiggsings to SU(3) separately.

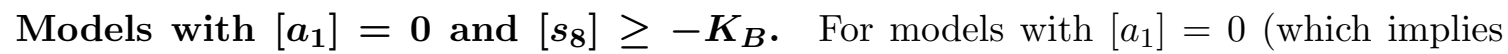
$\left[b_{1}\right] \geq 0$, yet allows us to put $b_{1} \equiv 0$ by shifting $\left.v \mapsto \frac{1}{a_{1}}\left(v-b_{1} w\right)\right)$ we have to demand $[t] \equiv\left[s_{8}\right] \geq-K_{B}$ in order to have an $\mathrm{SU}(3)$ with adjoints. The absence of the $\mathrm{SU}(2)$ factors in (5.1) further requires to consider models $X_{n+1}^{(0)}$ with $\left[a_{2}\right]=\left[a_{3}\right]=0$, as follows from (5.21). In addition, $\left[a_{i}\right]=0$ implies

$$
\left[b_{i}\right]=K_{B}+\left[s_{8}\right], \quad i=2,3,
$$

according to (3.17), which implies that the number of VEVs of adjoints of the $\mathrm{SU}(3)$ agrees with the number of monomials in $b_{2}, b_{3}$ that are tuned to zero for the unHiggsing.

Geometrically, as before the unHiggsing tranforms the generators of the Mordell-Weil group of $X_{n+1}$ into the Cartan divisors of the resolved $I_{3}^{\mathrm{s}}$-singularity. This is the analog of

\footnotetext{
${ }^{12}$ We note that models with $\left[a_{1}\right]>0$ and $\left[b_{1}\right]=0$, which implies $\left[s_{8}\right]<-K_{B}$ and $\left[s_{3}\right]>-K_{B}$, are equivalent upon $v \leftrightarrow w$ to models with $\left[a_{1}\right]=0$ and $\left[s_{8}\right]>-K_{B}$. Thus, we do not consider them here.
} 
the field theoretical statement that the two U(1)'s are embedded as the Cartan generators of the $\mathrm{SU}(3)$ in the unHiggsed theory.

The Weierstrass form of the models at hand is given by (5.25) and precisely agrees with the general Weierstrass model (B.19) with two $I_{2^{-}}$and one $I_{3}^{\mathrm{S}}$-singularities. If we set $\left[a_{2}\right]=\left[a_{3}\right]=0$ the $\mathrm{SU}(2)$ 's disappear and we simply recover the most general model with one $I_{3}^{\mathrm{s}}$-singularity in (B.11) upon identifying $t=s_{8}$. Thus, by unHiggsing the considered Abelian models defined by $X_{n+1}$ we reproduce the list of all F-theory models with one $I_{3}^{\mathrm{s}}$-singularity on a smooth divisor $t$.

Models with $\left[\boldsymbol{a}_{\mathbf{1}}\right],\left[\boldsymbol{b}_{\mathbf{1}}\right]>\mathbf{0}$. We begin by noting that the class $[t]$ is automatically effective and satisfies $[t] \geq\left[-K_{B}\right]$ as long as the term $a_{1} b_{1} s_{6} \subset t$ is present, which is the case if both $a_{1}$ and $b_{1}$ are non-trivial sections. ${ }^{13}$ Thus, we see that the $\mathrm{SU}(3)$ on $t=0$ always has adjoints that can be used to Higgs the theory back to a model with $\mathrm{U}(1)^{2}$ gauge symmetry. We recall that the divisor $t=0$ has ordinary double point singularities that support symmetric plus anti-symmetric representations, as shown in section 5.7.

If we further assume that $\left[\lambda_{1}\right]=\left[\lambda_{2}\right]=0$ are both trivial the gauge group of the tuned model $X_{n+1}^{(0)}$ is just to $\mathrm{SU}(3)$. Then, we have a clear embedding of the two U(1)'s into the maximal torus of $\mathrm{SU}(3)$. We observe that for $\left[\lambda_{1}\right]=\left[\lambda_{2}\right]=0$ we have $\left[a_{1}\right]=\left[a_{2}\right]=\left[a_{3}\right]$. Thus, the degrees of the sections $\Delta_{12}$ and $\Delta_{13}$ defined in (5.2) and (5.13), respectively, are equal. The number of monomials tuned to zero in the unHiggsing by imposing $\Delta_{12}, \Delta_{13} \rightarrow$ 0 is given e.g. for $B_{n}=\mathbb{P}^{2}$ by

$$
\left(\left[a_{1}\right]+2\right)\left(\left[a_{1}\right]+1\right)+\left(\left[a_{1}\right]+\left[s_{8}\right]-1\right)\left(\left[a_{1}\right]+\left[s_{8}\right]-2\right)-2=2\left[a_{1}\right]\left(\left[a_{1}\right]+\left[s_{8}\right]\right)+\left[s_{8}\right]\left(\left[s_{8}\right]-3\right)+2
$$

This agrees precisely with the number of VEVs of the $2 x_{(\mathbf{1}, \mathbf{1}, \mathbf{8})}$ in table 2 , where the factor of 2 is included due to the rank of $\mathrm{SU}(3)$.

The Weierstrass model we obtain by unHiggsing is given by $(5.22)$ for $\lambda_{1}=\lambda_{2}=1$. As pointed out earlier, it deviates from the standard form of an $I_{3}^{\mathrm{s}}$ singularity but still yields a model with $\mathrm{SU}(3)$ gauge group as shown in section 5.7. We note that at the current stage it is unknown whether every Calabi-Yau elliptic fibration with $I_{3}^{\mathrm{s}}$ singularities over a divisor $t=0$ with ordinary double points can be written as the Weierstrass model (5.22) or whether unHiggsing $X_{n+1}$ reproduces every model of the form (5.22).

We emphasize that the unHiggsed theory has two discrete degrees of freedom. These two parameters are the divisor classes $\left[s_{8}\right]$ and $\left[a_{1}\right]=\left[a_{2}\right]=\left[a_{3}\right]$. The latter sets the number of ordinary double point singularities of $t=0$. Geometrically, the appearance of the extra parameter is expected due to the special structure of the Weierstrass model (5.22) which is key to the presence of an $\mathrm{SU}(3)$ gauge group. In contrast, if we remove the additional substructure of $t=0$ by imposing $\left[a_{1}\right]=0$ we are left with only one parameter $\left[s_{8}\right]$, which is the class of the $t$ supporting the $\mathrm{SU}(3)$, as expected.

\footnotetext{
${ }^{13}$ If e.g. $a_{1}$ is constant the term involving $s_{6}$ in $t$ can be removed by redefining $s_{8}$ and we are back in a situation with $b_{1}=0$.
} 


\subsubsection{UnHiggsing from matching of spectra}

Again, we can rediscover those Calabi-Yau manifolds $X_{n+1}$ which arise from unHiggsing to an $\mathrm{SU}(3)$ with adjoints from the point of view of the matter spectrum of Abelian theory.

First we assume that there are no matter fields in the representation $(-2,-2)$ in table 2. Then, we recall from section 2.2.2 that an Abelian model with two U(1)'s which arises from a broken $\mathrm{SU}(3)$ has to have the charge lattice shown in figure 2. Notably, matter with charges $(2,0),(0,2)$ has to be absent while matter with charges $(2,1),(1,2)$ and $(-1,1)$ should generically be present. The unique solution to this is given by $\left[a_{1}\right]=\left[a_{2}\right]=\left[a_{3}\right]=0$, as follows from table 2 . We note that the remaining multiplicities automatically assume the form

$$
\begin{aligned}
& x_{(2,1)}=x_{(1,2)}=x_{(-1,1)}=\left[s_{8}\right] \cdot\left(\left[s_{8}\right]+K_{B}\right)=2 x_{\mathbf{8}}-2, \\
& x_{(1,0)}=x_{(0,1)}=x_{(1,1)}=-3\left[s_{8}\right] \cdot\left(3 K_{B}+\left[s_{8}\right]\right)=x_{\mathbf{3}},
\end{aligned}
$$

which implies that the matter spectrum falls into representations of $\mathrm{SU}(3)$ if we unHiggs by $b_{2}, b_{3} \rightarrow 0$. Here, we denote the multiplicity of an $\mathrm{SU}(3)$ representation $\mathbf{R}$ by $x_{\mathbf{R}}$. Indeed, the relations (6.10) readily follow from the branching rules of representations in an adjoint Higgsing given in section 2.2.2 and a comparison between tables 5 and 3. Note that we have to subtract one adjoint as it is eaten up by the massive gauge bosons. We can also obtain the relations (6.10) formally from the general relations (5.32) by dropping all multiplicities of $\mathrm{SU}(2) \times \mathrm{SU}(2)$ representations.

In order to include matter fields in the representation $(-2,-2)$ all we have to do is to relax the condition of a vanishing multiplicity $x_{(-2,-2)}$. As the discussion in section 2.2.4 shows, the only source for this representation is the symmetric representation of $\mathrm{SU}(3)$. The branching (2.10) of the symmetric suggests that we have to demand $x_{(-2,-2)}=x_{(-2,0)}=$ $x_{(0,-2)}$. A unique solution to this in a natural parametrization is given by

$$
\left[a_{1}\right]=\left[a_{2}\right]=\left[a_{3}\right], \quad\left[s_{8}\right]=[t]-2\left[a_{1}\right]
$$

for an effective class $[t]$. Using this relation we clearly reproduce the relations (5.32) after setting the multiplicities of all $\mathrm{SU}(2)$-representations to zero. Thus, in an unHiggsing $t$ plays the role of the divisor class supporting the $\mathrm{SU}(3)$ and $\left[a_{1}\right]$ is related to the number of symmetric representations. The entire spectrum of the Abelian theory falls into representations of $\mathrm{SU}(3)$.

\subsubsection{Examples: models on $\mathbb{P}^{2}$}

We conclude by constructing explicit models for the base $B_{2}=\mathbb{P}^{2}$. We start with an Abelian model specified by the CY-threefold $X_{3}$, solve the effectiveness conditions (3.17) and impose the conditions for an enhancement to $\mathrm{SU}(3)$. Then we tune the complex 
structure in order to unHiggs to $X_{3}^{(0)}$. We obtain the following list of models:

\begin{tabular}{|c|c||c|c|c|c|c|c||c|}
\hline$\left[a_{i}\right]$ & {$\left[s_{8}\right]$} & $b_{i}$ & $s_{1}$ & $s_{2}$ & $s_{3}$ & $s_{5}$ & $s_{6}$ & {$[t]$} \\
\hline 0 & 6 & 3 & 0 & 0 & 0 & 3 & 3 & 6 \\
0 & 5 & 2 & 3 & 2 & 1 & 4 & 3 & 5 \\
0 & 4 & 1 & 6 & 4 & 2 & 5 & 3 & 4 \\
0 & 3 & 0 & 9 & 6 & 3 & 6 & 3 & 3 \\
\hline \hline 1 & 4 & 2 & 0 & 1 & 2 & 2 & 3 & 6 \\
1 & 3 & 1 & 3 & 3 & 3 & 3 & 3 & 5 \\
\hline
\end{tabular}

We note that the first four lines reproduce precisely the list of models with a standard $I_{3}^{\mathrm{S}}$ singularity on a smooth divisor found in (B.16), where we identify $t=s_{8}$. The last two lines are models corresponding to the two possible solutions to the condition that $\left[s_{1}\right]$ is effective when the divisor $t$ is of the form (5.10) with an ordinary double point. Indeed, for $\left[\lambda_{1}\right]=\left[\lambda_{2}\right]=0$ the effectiveness constraint on $\left[s_{1}\right]$ reduces to

$$
3[t]+\left[s_{1}\right]=18
$$

which is solved only by $[t]=\left[a_{1}\right]+\left[b_{1}\right]+3=5,6$ with $\left[s_{8}\right]=3,4$ and $\left[a_{1}\right]=\left[b_{1}\right]=1$ or $\left[a_{1}\right]=\left[b_{1}\right]-1=1$, respectively. ${ }^{14}$

Again, we emphasize that the new Calabi-Yau manifolds $X_{n+1}$ are needed to have a geometrical description in F-theory of the Higgs branch of an SU(3) gauge theory broken by adjoints to two U(1)'s. The relevant Abelian theories can not be obtained from the $d P_{2}$-elliptic fibrations in [8-12], unless $\left[s_{8}\right]=3$.

There are some caveats regarding the completeness of our construction of models with an $\mathrm{SU}(3)$ on a divisor with double points in $\mathbb{P}^{2}$. As we have seen in (6.12), we only obtain models with one or two double points on a quintic or a sextic, respectively. However, we expect that models on $\mathbb{P}^{2}$ with more double points or an $\mathrm{SU}(3)$ divisor of a lesser degree exist. In particular, we expect the existence of an $\mathrm{SU}(3)$ on a quartic with one adjoint and up to two double points, on a quintic with an adjoint and up to five double points and on a sextic with an adjoint and up to nine ordinary double points. On a technical level, the reason for the limitations of our model is the term $b_{1} a_{1} s_{6}$ in $t$, which imposes the condition $[t]-3=\left[a_{1}\right]+\left[b_{1}\right]$ fixing the number of double points. Currently, we do not know of any geometrical or physical reason for this constraint, which is intrinsic to our constructions, to hold universally, or how to see such a constraint from the low-energy theory.

\subsection{Unhiggsing of two $\mathrm{U}(1)$ 's to $\mathrm{SU}(2) \times \mathrm{SU}(2) \times \mathrm{SU}(3)$}

Finally, we consider Abelian F-theory models with two U(1)'s that unHiggs to a model $X_{n+1}^{(0)}$ in which the full non-Abelian gauge group $G_{\text {uni }}=\mathrm{SU}(2) \times \mathrm{SU}(2) \times \mathrm{SU}(3)$ is realized. In this case, the two U(1)'s are embedded diagonally into the Cartan subalgebra of $G_{\text {uni }}$.

\footnotetext{
${ }^{14}$ Note that there exists a model with $\left[a_{1}\right]-1=\left[b_{1}\right]=1$ and $\left[s_{8}\right]=2$. It is mapped to the model $\left[a_{1}\right]=\left[b_{1}\right]-1=1$ and $\left[s_{8}\right]=4$ using the symmetry $v \leftrightarrow v$.
} 
Depending on the discrete parameters specifying $X_{n+1}$, the Abelian theory can be obtained from the non-Abelian one by different Higgsings. While all Abelian models considered here arise from breaking $G_{\text {uni }}$ by the bifundamentals $(\mathbf{2}, \mathbf{1}, \mathbf{3})$ and $(\mathbf{1}, \mathbf{2}, \mathbf{3})$,

$$
\mathrm{U}(1) \times \mathrm{U}(1) \stackrel{\text { bifunds. }}{\longleftrightarrow} \mathrm{SU}(2) \times \mathrm{SU}(2) \times \mathrm{SU}(3)
$$

certain models admit an alternative Higgsing via adjoints and fundamentals. This alternative Higgsing can be viewed as a two-stage version of the bifundamental Higgsing. It illuminates the geometrical process which turns a vertical into a horizontal divisor, i.e. a section into a Cartan divisor. Thus, we will discuss it in detail.

There are four different types of models $X_{n+1}$ that unHiggs to $G_{\text {uni }}$. They are

- specialized models $\left(\left[a_{1}\right]=0\right)$ with $\left[a_{2}\right],\left[a_{3}\right] \geq-K_{B}$, as in section 6.1 , but with $\left[s_{8}\right]>0$. The Higgsing alternative to (6.14) is by adjoints of the two SU(2)'s and a consecutive Higgsing by fundamentals of the residual SU(3) with appropriate $\mathrm{U}(1) \times \mathrm{U}(1)$ charges.

- specialized models with $\left[s_{8}\right] \geq-K_{B}$, as in section 6.2 , but without $\left[a_{2}\right]=\left[a_{3}\right]=0$ and models with $\left[a_{1}\right],\left[b_{1}\right]>0$, again as in section 6.2 , but relaxing $\left[\lambda_{1}\right]=\left[\lambda_{2}\right]=0$. As an alternative to (6.14) we can Higgs by adjoints of the $\mathrm{SU}(3)$ and break the residual $\mathrm{SU}(2) \times \mathrm{SU}(2)$ by fundamentals with $\mathrm{U}(1) \times \mathrm{U}(1)$ charges.

- specialized models with $\left[a_{2}\right]<-K_{B}$ but $\left[a_{3}\right] \geq-K_{B}$ or vice versa and $\left[s_{8}\right]<$ $-K_{B}$. In this case we can Higgs one $\mathrm{SU}(2)$ with adjoints, then Higgs the remaining $\mathrm{SU}(2) \times \mathrm{SU}(3)$ by a bifundamental and finally Higgs the residual $\mathrm{SU}(2)$ by a fundamental with non-trivial U(1) charges.

- specialized models with $\left[a_{2}\right],\left[a_{3}\right]<-K_{B}$ and $\left[s_{8}\right]<-K_{B}$. In this case, there is no alternative Higgsing to (6.14).

We note that some non-Abelian theories defined by $X_{n+1}^{(0)}$ have Higgs branches parameterized by Higgses in the bifundamental representation $(\mathbf{2}, \mathbf{2}, \mathbf{1})$, different fundamentals (with trivial $\mathrm{U}(1)$ charges) and with other final gauge symmetries. The analysis of these branches is beyond the scope of this article and is left for future works.

\subsubsection{Geometric discussion \& comparison to field theory}

We will analyze the four different types of models in the order outlined above.

Models with $\left[a_{1}\right]=0,\left[a_{2}\right],\left[a_{3}\right] \geq-K_{B}$ and $\left[s_{8}\right]>0$. These models are analyzed similarly to those discussed in section 6.1 , however relaxing the condition $\left[s_{8}\right]=0$. In this case, the tuning $b_{2}, b_{3} \rightarrow 0$ unHiggsing the $\mathrm{U}(1)$ 's produces the model $X_{n+1}^{(0)}$. First, by tuning $b_{2} \equiv 0$ we get the gauge group $\mathrm{SU}(2) \times \mathrm{SU}(2) \times \mathrm{U}(1)$, cf. the discussion before (5.12). Then, we get the full gauge group $G_{\text {uni }}$ if we also set $b_{3} \equiv 0$ as shown before (5.18). The Weierstrass model of $X_{n+1}^{(0)}$ is given by (5.22). 
In order to understand the embedding of the U(1)'s into the Cartan subalgebra of $G_{\text {uni }}$ from a geometric point of view we note that $\left[s_{8}\right]>0$, using (3.19), implies

$$
\left[b_{i}\right] \geq\left[s_{8}\right], \quad i=2,3 .
$$

Thus, the degree of the $b_{i}$ is larger than that of $s_{8}$. Thus, we can tune the complex structure of $X_{n+1}$ so that

$$
b_{2}=s_{8} b_{2}^{\prime}, \quad b_{3}=s_{8} b_{3}^{\prime}
$$

for appropriate sections $b_{2}^{\prime}$ and $b_{3}^{\prime}$. We obtain an F-theory model with two U(1)'s and an extra $I_{3}^{\mathrm{s}}$-singularity at $s_{8}=0$, as can be seen by inspecting of the Weierstrass form (3.11) with (A.1), (A.2) for $X_{n+1}$. Thus, the $\mathrm{SU}(3)$ gauge group at $s_{8}$ can already be induced prior to unHiggsing the U(1)'s in the Abelian model. The U(1)'s are unHiggsed into two $\mathrm{SU}(2)$ 's by tuning $b_{2}^{\prime}=b_{3}^{\prime} \equiv 0$ corresponding to an adjoint Higgsing as in section 6.1.

The field theoretic Higgsing corresponding to the intermediate tuning (6.16) can be inferred by noting that the U(1) charges of the theory are changed. Indeed, geometrically, the Shioda map is altered due to the presence of the Cartan divisors of the SU(3) [55, 57]. This change shifts the U(1) charges of all matter fields in such a way that the new spectrum of the theory after the tuning (6.16) is of the form of figure 1, while the original smooth model $X_{n+1}$ has a spectrum like in figure 3 . Thus, the field-theoretical analogue of (6.16) has to be a Higgsing by fundamentals $\mathbf{3}_{\left(q_{1}, q_{2}\right)}$ with appropriate $\mathrm{U}(1)$ charges $\left(q_{1}, q_{2}\right)$. The correct $\mathrm{U}(1)$ charges are identified by starting with the spectrum in table 5 of the unHiggsed model $X_{n+1}^{(0)}$. Then, we Higgs the two SU(2)'s by adjoints and identify the corresponding triplets $\mathbf{3}_{\left(q_{1}, q_{2}\right)}$ by the requirement that the resulting spectrum matches table 3 . We find that the correct states are precisely the triplets $\mathbf{3}_{(-1,0)}$ and $\mathbf{3}_{(0,1)}$ that are inside the bifundamentals $(\mathbf{2}, \mathbf{1}, \mathbf{3})$ and $(\mathbf{1}, \mathbf{2}, \mathbf{3})$, respectively. Thus, the $\mathrm{U}(1)$-generators of $X_{n+1}$ are embedded into the Cartans of $G_{\text {uni }}$ as in (2.6), i.e. the bifundamental Higgsings.

We note that the spectrum of the Abelian theory obeys the relations (5.32) with $x_{(-2,-2)}=0$. It is important to emphasize that these relations are simultaneously compatible with a Higgsing by bifundamentals as in (6.14) and by a combination of adjoint and fundamental Higgses, as just described.

We summarize the chain of Higgsings that are possible if we invoke the tunings (6.16) in the following diagram:

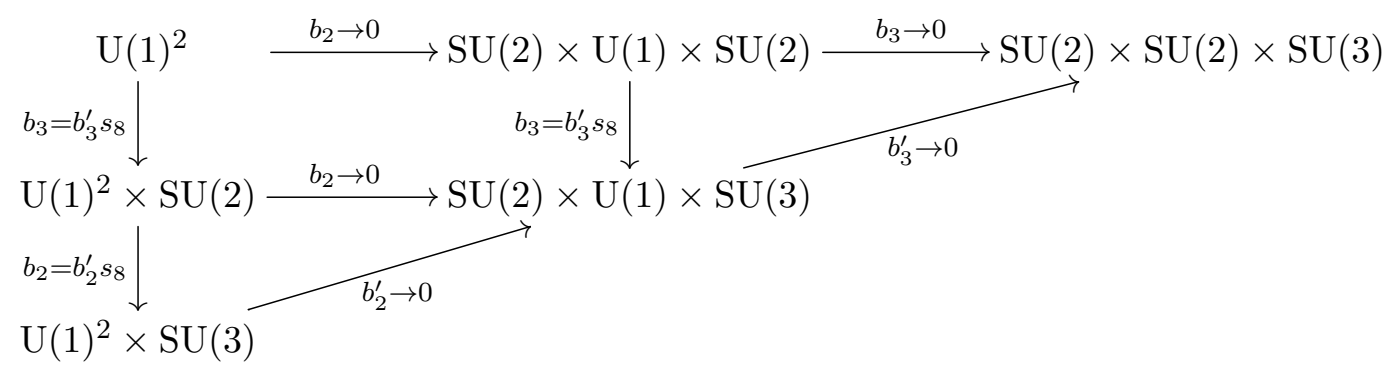

Here we indicate the relevant tunings of the complex structure of the Calabi-Yau manifold $X_{n+1}$ next to the respective arrow. The first line of (6.17) is identical to (6.14). Vertical arrows correspond to a Higgsing by a fundamental with non-trivial U(1) charges, horizontal 
arrows are bifundamental Higgsings and diagonal arrows are $\mathrm{SU}(2)$ adjoint Higgsings. The latter correspond to tunings that unHiggs U(1)'s into Cartan generators of SU(2)'s.

We note that starting from the non-Abelian theory $X_{n+1}^{(0)}$ there are other possible Higgs branches. Higgsing by the bifundamental $(\mathbf{2}, \mathbf{2}, \mathbf{1})$ leads to a theory with just one $\mathrm{U}(1)$, while Higgsings by fundamentals of SU(2)'s lead to a theory with no residual Abelian gauge group. These Higgsings are not considered here, as they are not related to the Abelian model $X_{n+1}$.

Models with $\left[a_{1}\right]=0$ and $\left[s_{8}\right] \geq-K_{B}$ or $\left[a_{1}\right],\left[b_{1}\right]>0$. These models are analyzed in analogy to those in section 6.2. However, the full gauge group $G_{\text {uni }}$ is realized as can be seen e.g. from the Weierstrass model given by (5.25). Indeed, models with $\left[a_{1}\right]=0$ are unHiggsed by tuning $b_{2}, b_{3} \rightarrow 0$ to $X_{n+1}^{(0)}$ also in the case if the conditions $\left[a_{2}\right]=\left[a_{3}\right]=0$ are relaxed. Similarly, models with $\left[a_{1}\right],\left[b_{1}\right]>0$ are unHiggsed by imposing (5.2) and (5.13). This is solved, assuming $\left[a_{2}\right]-\left[a_{1}\right] \geq 0,\left[a_{3}\right]-\left[a_{1}\right] \geq 0$, by (5.4) and (5.14) with non-trivial $\left[\lambda_{1}\right]$ and $\left[\lambda_{2}\right]$. In other cases, we are in situation $\left.\mathbf{D}\right)$ in section 5.2 , which can be made equivalent to a case with $\left[a_{1}\right]=0$ by setting $b_{1}=0$, as discussed there. We assume in the rest of this section that we are in the situation where $\left[a_{2}\right]-\left[a_{1}\right] \geq 0,\left[a_{3}\right]-\left[a_{1}\right] \geq 0$ applies.

Geometrically, we understand the embedding of the U(1)s into the maximal torus of $G_{\text {uni }}$ by noting that the condition $\left[s_{8}\right] \geq-K_{B}$ implies, by virtue of (3.19), that

$$
\left[b_{i}\right] \geq\left[a_{i}\right], \quad i=1,2,3,
$$

i.e. the degrees of the $b_{i}$ are larger than that of the corresponding $a_{i}$. Thus, we can tune the complex structure of $X_{n+1}$ so that

$$
b_{2}=a_{2} b_{2}^{\prime}, \quad b_{3}=a_{3} b_{3}^{\prime},
$$

for appropriate sections $b_{2}^{\prime}, b_{3}^{\prime}$. The resulting F-theory models still have two rational sections generating a rank two MW-group but also have $I_{2}$-singularities at $a_{2}=0$ and $a_{3}=0$. In fact, the resulting elliptic fibration is described by

$$
p=u f_{u}+a_{2} a_{3} v\left(v+b_{2}^{\prime} w\right)\left(v+b_{3}^{\prime} w\right),
$$

which clearly has $I_{2}$ singularities at $a_{2}=0$ and $a_{3}=0$, yet has three distinct rational points. The relevant tunings that reduce the MW-rank are then given by $b_{2}^{\prime}, b_{3}^{\prime} \rightarrow 0$, which creates the $I_{3}^{\mathrm{s}}$ singularity at $s_{8}=0$.

Analogously, a general model $X_{n+1}$ with $\left[a_{1}\right],\left[b_{1}\right]>0$ can be tuned by setting

$$
a_{i}=\lambda_{i-1} a_{i}^{\prime}, \quad b_{i}=\lambda_{i-1} b_{i}^{\prime}
$$

for appropriate sections $a_{i}^{\prime}, b_{i}^{\prime}$ for $i=2,3$ with $\left[a_{2}^{\prime}\right]=\left[a_{1}\right]$ and $\left[a_{3}^{\prime}\right]=\left[a_{1}\right]$. The CYconstraint (3.4) then assumes the form

$$
p=u f_{u}(u, v, w)+\lambda_{1} \lambda_{2}\left(a_{1} v+b_{1} w\right)\left(a_{2}^{\prime} v+b_{2}^{\prime} w\right)\left(a_{3}^{\prime} v+b_{3}^{\prime} w\right),
$$

so we see that the tuning (6.21) maintains all rational sections, but introduces already $I_{2}$ singularities corresponding to the $\mathrm{SU}(2)$ 's at $\lambda_{1}=0$ and $\lambda_{2}=0$. In this case, the tunings 
that unHiggs the $\mathrm{U}(1)$ 's to the $\mathrm{SU}(3)$ associated to an $I_{3}^{\mathrm{s}}$ singularity at $t=0$, cf. section 5.7, are given by

$$
a_{1} b_{i}^{\prime}-b_{1} a_{i}^{\prime} \equiv 0
$$

for both $i=2,3$ yielding the CY-manifold $X_{n+1}^{(0)}$ with gauge group $G_{\text {uni }}$.

In field theory, the intermediate tunings (6.19), (6.21) corresponds to a Higgsing by fundamentals of the SU(2)'s which carry non-trivial U(1)-charges. Again, this is clear from the change in the Shioda map of the rational sections due to the $\mathrm{SU}(2)$ 's. The correct matter fields are in the representations $(\mathbf{2}, \mathbf{1})_{(1,0)}$ and $(\mathbf{1}, \mathbf{2})_{(0,-1)}$ that originate from the bifundamentals $(\mathbf{2}, \mathbf{1}, \mathbf{3})$ and $(\mathbf{1}, \mathbf{2}, \mathbf{3})$, respectively, so that the $\mathrm{U}(1)$ 's are embedded via (2.6) into the Cartan generators of $G_{\text {uni }}$.

The spectrum of the Abelian theories obtained from models $X_{n+1}$ with $\left[a_{1}\right]=0$ obey the relations $(5.32)$ with $x_{(-2,-2)}=0$ while models with $\left[a_{1}\right],\left[b_{1}\right]>0$ allow for a non-zero multiplicity $x_{(-2,-2)}$. We note again that the relations (5.32) are compatible both with a Higgsing by bifundamentals or by adjoints and fundamentals, as the one just described.

We summarize the chain of Higgsings we just discussed that also allows for the tunings (6.19) in the following diagram:

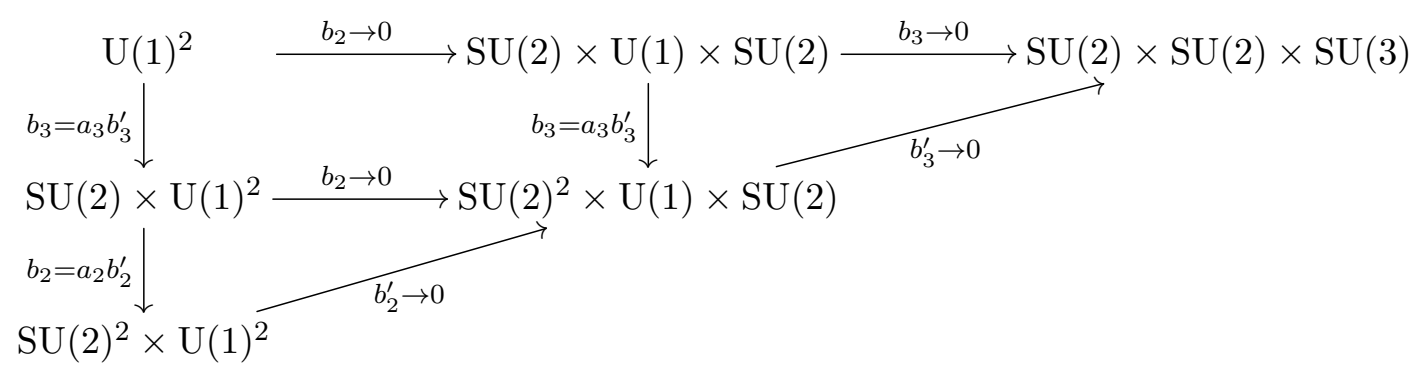

Here the relevant tunings of the complex structure of the Calabi-Yau manifold $X_{n+1}$ are indicated next to the respective arrow. The first line of (6.24) is the Higgsing in (6.14). Vertical arrows correspond to a Higgsing by a fundamental with non-trivial U(1) charges, horizontal arrows are bifundamental Higgsings and diagonal arrows are adjoint Higgsings of the $\mathrm{SU}(3)$. The latter correspond to tunings that unHiggs U(1)'s into Cartan generators of $\mathrm{SU}(3)$ 's.

We emphasize that starting from the non-Abelian theory $X_{n+1}^{(0)}$ there are other possible Higgs branches. Higgsing by the bifundamental $(\mathbf{2}, \mathbf{2}, \mathbf{1})$ and then by the adjoints of SU(3) leads to a theory with three U(1)'s, while Higgsings by fundamentals of SU(3)'s and then by the bifundamental of the $\mathrm{SU}(2)$ 's leads to a theory with one Abelian gauge group. These Higgsings are not considered here as they are not relevant for obtaining the Abelian model $X_{n+1}$.

We conclude with the observation that the effectiveness condition (6.2) implies that whenever $[t] \geq-K_{B}$ the model can at most have one $\mathrm{SU}(2)$ with an adjoint. Consequently there are models with adjoints for one $\mathrm{SU}(2)$ and the $\mathrm{SU}(3)$. These models exhibit an adjoint Higgsing to a theory with $\mathrm{U}(1)^{3} \times \mathrm{SU}(2)$ gauge group.

Models with $\left[a_{1}\right]=0,\left[a_{2}\right]<-K_{B},\left[a_{3}\right] \geq-K_{B}$ and $\left[s_{8}\right]<-K_{B}$. The main distinction of the following discussion from the above is that the CY-manifolds $X_{n+1}$ con- 
sidered here do not yield non-Abelian theories with adjoints of rank two gauge groups. In fact, for models with $\left[a_{1}\right]=0,\left[s_{8}\right]<-K_{B}$ and $\left[a_{2}\right]<-K_{B},\left[a_{3}\right] \geq-K_{B}$ only one $\mathrm{SU}(2)$ in $X_{n+1}^{(0)}$ carries adjoints. Thus, we can Higgs the theory on this adjoint yielding one $\mathrm{U}(1)$. The remaining non-Abelian group $\mathrm{SU}(2) \times \mathrm{SU}(3)$ is Higgsed on a bifundamental to $\mathrm{SU}(2) \times \mathrm{U}(1)$ and then on a charged fundamental to $\mathrm{U}(1)$. As before the spectrum of the Abelian and the non-Abelian theory obey the relations (5.32). Geometrically, there exists only one intermediate tuning

$$
b_{3}=b_{3}^{\prime} s_{8},
$$

for appropriate $b_{3}^{\prime}$ which creates an $\mathrm{SU}(2)$ on $s_{8}=0$ prior to unHiggsing any $\mathrm{U}(1)$.

The chain of (un-)Higgsings possible in this model is given by

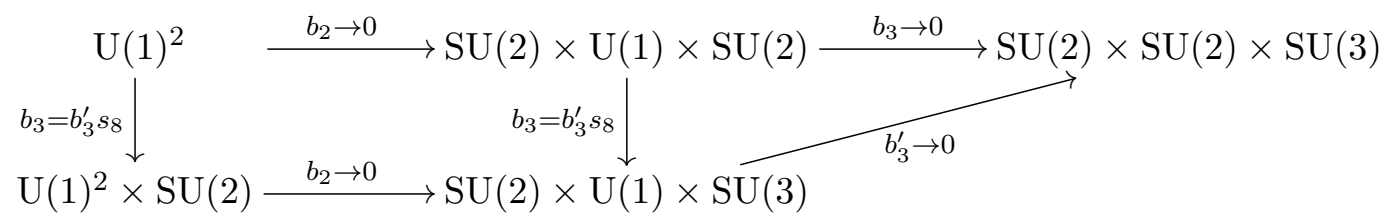

As before the relevant tunings of the complex structure of $X_{n+1}$ are indicated next to the respective arrow, vertical arrows correspond to Higgsings by charged fundamentals, horizontal arrows are Higgsings by bifundamentals and diagonal arrows are adjoint Higgsings.

We note that the number of alternative Higgsings of the non-Abelian theory defined by $X_{n+1}^{(0)}$ is limited. All Higgsings alternative to (6.26) yield theories with one or no U(1).

Models with $\left[a_{1}\right]=0$ and $\left[a_{2}\right],\left[a_{3}\right],\left[s_{8}\right]<-K_{B}$. For these models no intermediate tuning (6.16) of $X_{n+1}$ is possible. Thus, the unHiggsing of the Abelian sector is induced by $b_{2}, b_{3} \rightarrow 0$ and cannot be split into steps. We obtain a non-Abelian model described by $X_{n+1}^{(0)}$ with gauge group $G_{\text {uni }}$ with a spectrum related to that of the Abelian model via the relations (5.32). The only (un-)Higgsing chain possible is the one in (6.14):

$$
\mathrm{U}(1)^{2} \stackrel{b_{2} \rightarrow 0}{\longrightarrow} \mathrm{SU}(2) \times \mathrm{U}(1) \times \mathrm{SU}(2) \stackrel{b_{3} \rightarrow 0}{\longrightarrow} \mathrm{SU}(2) \times \mathrm{SU}(2) \times \mathrm{SU}(3)
$$

\subsubsection{Examples: models on $\mathbb{P}^{2}$}

We conclude the discussion of models with an unHiggsing to a non-Abelian theory with the full gauge group $G_{\text {uni }}$ (or in special cases $\mathrm{SU}(2) \times \mathrm{SU}(3)$, when one $\mathrm{SU}(2)$ is on a trivial divisor) by constructing explicit models for the base $B_{2}=\mathbb{P}^{2}$. As before, we start with an Abelian F-theory model specified by the threefold $X_{3}^{(2)}$ which is in one of the four classes of models discussed in this section. Then we tune the model appropriately to the threefold $X_{3}^{(0)}$. We discuss the four different classes of models separately. Note again that we assume in this discussion that we are in situation $\mathbf{A}$ ), of the possibilities distinguished above.

The list of models $X_{3}$ on $B_{2}=\mathbb{P}^{2}$ with $\left[a_{1}\right]=0,\left[a_{2}\right],\left[a_{3}\right] \geq 3$ and $\left[s_{8}\right]>0$ is given by:

\begin{tabular}{|c|c||c|c|c|c|c|c|c|c|}
\hline$\left[a_{2}\right]$ & {$\left[a_{3}\right]$} & $b_{2}$ & $b_{3}$ & $s_{1}$ & $s_{2}$ & $s_{3}$ & $s_{5}$ & $s_{6}$ & $s_{8}$ \\
\hline 3 & 3 & 1 & 1 & 3 & 4 & 5 & 2 & 3 & 1 \\
3 & 3 & 2 & 2 & 0 & 2 & 4 & 1 & 3 & 2 \\
3 & 4 & 1 & 2 & 1 & 3 & 5 & 1 & 3 & 1 \\
\hline
\end{tabular}


These three models are all the models in the list (B.21) of theories with $\mathrm{SU}(2) \times \mathrm{SU}(2) \times$ $\mathrm{SU}(3)$ gauge group over $B_{2}=\mathbb{P}^{2}$ that meet the requirement $\left[a_{2}\right],\left[a_{3}\right] \geq 3$, i.e. have $\mathrm{SU}(2) \times \mathrm{SU}(2)$ with adjoints. Thus, we have reproduced the complete list of such models by unHiggsing the Abelian theories defined by $X_{3}$.

The list of models $X_{3}$ on $B_{2}=\mathbb{P}^{2}$ with $\left[s_{8}\right] \geq 3$ and $\left[a_{2}\right]$, or $\left[a_{3}\right] \geq 0$ reads:

\begin{tabular}{|c|c||c|c|c|c|c|c|c|c|}
\hline$\left[a_{2}\right]$ & {$\left[a_{3}\right]$} & $b_{2}$ & $b_{3}$ & $s_{1}$ & $s_{2}$ & $s_{3}$ & $s_{5}$ & $s_{6}$ & $s_{8}$ \\
\hline 0 & 1 & 2 & 3 & 1 & 1 & 1 & 3 & 3 & 5 \\
0 & 1 & 1 & 2 & 4 & 3 & 2 & 4 & 3 & 4 \\
0 & 1 & 0 & 1 & 7 & 5 & 3 & 5 & 3 & 3 \\
0 & 3 & 1 & 4 & 0 & 1 & 2 & 2 & 3 & 4 \\
0 & 3 & 0 & 3 & 3 & 3 & 3 & 3 & 3 & 3 \\
0 & 2 & 1 & 3 & 2 & 2 & 2 & 3 & 3 & 4 \\
0 & 2 & 0 & 2 & 5 & 4 & 3 & 4 & 3 & 3 \\
0 & 4 & 0 & 4 & 1 & 2 & 3 & 2 & 3 & 3 \\
1 & 1 & 1 & 1 & 5 & 4 & 3 & 4 & 3 & 3 \\
1 & 1 & 2 & 2 & 2 & 2 & 2 & 3 & 3 & 4 \\
1 & 2 & 1 & 2 & 3 & 3 & 3 & 3 & 3 & 3 \\
1 & 2 & 2 & 3 & 0 & 1 & 2 & 2 & 3 & 4 \\
1 & 3 & 1 & 3 & 1 & 2 & 3 & 2 & 3 & 3 \\
2 & 2 & 2 & 2 & 1 & 2 & 3 & 2 & 3 & 3 \\
\hline
\end{tabular}

Again, we reproduce all models in the list in (B.21) of theories with $\mathrm{SU}(2) \times \mathrm{SU}(2) \times \mathrm{SU}(3)$ gauge group over $B_{2}=\mathbb{P}^{2}$ that meet the requirement of having an $\mathrm{SU}(3)$ with adjoints.

The number of models $X_{3}$ on $B_{2}=\mathbb{P}^{2}$ with $\left[a_{1}\right],\left[b_{1}\right]>0$ is very limited. Indeed, by inspection of the last term of $g$ in (5.22), we obtain the equality

$$
-6 K_{B}-2\left[\lambda_{1}\right]-2\left[\lambda_{2}\right]-3[t]=\left[s_{1}\right] \geq 0,
$$

which agrees precisely with the condition (6.2) we have derived from the Abelian model $X_{n+1}$. This condition is very constraining as both classes $\left[a_{1}\right]$ and $\left[b_{1}\right]$ have to be strictly non-zero so that $[t]=3+\left[a_{1}\right]+\left[b_{1}\right] \geq 5$ on $\mathbb{P}^{2}$. The only solutions to it are $\left[a_{1}\right]=\left[b_{1}\right]=1$ for $[t]=5$, which allows for $\left[\lambda_{1}\right]=\left[\lambda_{2}\right]=0$ or $\left[\lambda_{1}\right]=0,\left[\lambda_{2}\right]=1$, and $\left[a_{1}\right]=\left[b_{1}\right]-1=1$, which only allows for $\left[\lambda_{1}\right]=\left[\lambda_{2}\right]=0$. Thus, we only obtain one solution with one $\operatorname{SU}(2)$, in addition to the two solutions in (6.12):

\begin{tabular}{|c|c|c||c|c|c|c|c|c|c|c|c|}
\hline$\left[a_{1}\right]$ & {$\left[a_{2}\right]$} & {$\left[a_{3}\right]$} & $b_{1}$ & $b_{2}$ & $b_{3}$ & $s_{1}$ & $s_{2}$ & $s_{3}$ & $s_{5}$ & $s_{6}$ & $s_{8}$ \\
\hline 1 & 1 & 2 & 1 & 1 & 2 & 1 & 2 & 3 & 2 & 3 & 3 \\
\hline
\end{tabular}

This is a model with an $\mathrm{SU}(3)$ on a quintic in $\mathbb{P}^{2}$ with one ordinary double point singularity at $a_{1}=b_{1}=0$ and an $\mathrm{SU}(2)$ on a line $\lambda_{2}=0$. As discussed earlier we reproduce the full list of models compatible with the structure of Weierstrass models of the form (5.22) and divisor $t=0$ of the form (5.10). However, we currently do not know whether this is the complete list of models with an $\mathrm{SU}(3)$ on a divisor in $\mathbb{P}^{2}$ with ordinary double points that carry symmetric representations. 
The list of models $X_{3}$ on $B_{2}=\mathbb{P}^{2}$ with $\left[s_{8}\right]<3,\left[a_{3}\right] \geq 3$ and $\left[a_{2}\right]<3$ together with models having $\left[a_{2}\right],\left[a_{3}\right],\left[s_{8}\right]<3$ reads:

\begin{tabular}{|c|c||c|c|c|c|c|c|c|c|}
\hline \multicolumn{7}{|c|}{ Models with $\left[s_{8}\right]<3,\left[a_{3}\right] \geq 3$ and $\left[a_{2}\right]<3$} \\
\hline$\left[a_{2}\right]$ & {$\left[a_{3}\right]$} & $b_{2}$ & $b_{3}$ & $s_{1}$ & $s_{2}$ & $s_{3}$ & $s_{5}$ & $s_{6}$ & $s_{8}$ \\
\hline 1 & 3 & 0 & 2 & 4 & 4 & 4 & 3 & 3 & 2 \\
1 & 4 & 0 & 3 & 2 & 3 & 4 & 2 & 3 & 2 \\
1 & 5 & 0 & 4 & 0 & 2 & 4 & 1 & 3 & 2 \\
2 & 3 & 0 & 1 & 5 & 5 & 5 & 3 & 3 & 1 \\
2 & 3 & 1 & 2 & 2 & 3 & 4 & 2 & 3 & 2 \\
2 & 4 & 0 & 2 & 3 & 4 & 5 & 2 & 3 & 1 \\
2 & 4 & 1 & 3 & 0 & 2 & 4 & 1 & 3 & 2 \\
2 & 5 & 0 & 3 & 1 & 3 & 5 & 1 & 3 & 1 \\
\hline \multicolumn{7}{|c|}{ Models with $\left[a_{2}\right],\left[a_{3}\right],\left[s_{8}\right]<3$} \\
\hline 1 & 1 & 0 & 0 & 8 & 6 & 4 & 5 & 3 & 2 \\
1 & 2 & 0 & 1 & 6 & 5 & 4 & 4 & 3 & 2 \\
2 & 2 & 0 & 0 & 7 & 6 & 5 & 4 & 3 & 1 \\
2 & 2 & 1 & 1 & 4 & 4 & 4 & 3 & 3 & 2 \\
\hline
\end{tabular}

We note that this covers all remaining models in (B.21) that can be Higgsed supersymmetrically to an Abelian theory with two U(1)'s. There are models that do not admit such Higgsings, either because the realized non-Abelian gauge group is too small or because there are less than two matter fields in both bifundamental representations $(\mathbf{2}, \mathbf{1}, \mathbf{3})$ and $(\mathbf{1}, \mathbf{2}, \mathbf{3})$, respectively. In the latter case, a D-flat Higgsing is impossible. We note, though, that some of those models do have a Higgsing to a model with one U(1). It is satisfying that the effectiveness conditions (3.17) and (3.19) required by the existence of a smooth and generic model $X_{n+1}$ reproduce these field theory results.

\section{$7 \quad$ Further research directions}

While we believe that the model constructed in this paper gives a fairly complete picture of the most general classes of F-theory models that can be constructed with two Abelian factors $\mathrm{U}(1) \times \mathrm{U}(1)$, many of the results we have found suggest generalizations that may have much broader consequences for our understanding of F-theory models and the corresponding supergravity theories. In particular, the general structure of the unHiggsing patterns identified in the $\mathrm{U}(1)^{2}$ models considered here suggests that similar patterns may govern F-theory models with arbitrary numbers of Abelian factors $\mathrm{U}(1)^{k}$. Our construction has also given rise to an explicit realization of a class of models where a non-Abelian gauge group is supported on a singular curve, giving rise to a non-generic matter representation. In this section we look ahead to how these features may be generalized in future work.

\subsection{More U(1) factors}

In the most general class of $\mathrm{U}(1) \times \mathrm{U}(1)$ models that we have considered here, the charge spectrum (figure 4) can be identified with the set of charges arising when a non-Abelian 
group $\mathrm{SU}(2) \times \mathrm{SU}(2) \times \mathrm{SU}(3)$ is broken by Higgsing. As we have shown, this corresponds to the fact that the generic model can be "unHiggsed" by transforming the horizontal divisors (sections) associated with the two $\mathrm{U}(1)$ factors into vertical divisors in the total space of the Calabi-Yau that lie over divisors of the form $A C$ and $B C$ in the base of the elliptic fibration. Here, $C$ is a common factor of the two divisors, and picks up the rank two $\mathrm{SU}(3)$ non-Abelian group, while $A$ and $B$ support $\mathrm{SU}(2)$ gauge factors.

This suggests a natural structure for general models with more $U(1)$ factors. For three $\mathrm{U}(1)$ factors $\mathrm{U}(1) \times \mathrm{U}(1) \times \mathrm{U}(1)$, for example, we could imagine transforming all three horizontal divisors into vertical divisors. The most general structure for the divsors in the base that would support the three non-Abelian factors would be

$$
A_{1} B_{12} B_{13} C, A_{2} B_{12} B_{23} C, A_{3} B_{13} B_{23} C \text {. }
$$

Each $A_{i}$ divisor would then support an $\mathrm{SU}(2)$ factor, each $B_{i j}$ divisor would support an $\mathrm{SU}(3)$ factor, and $C$ would support a rank three factor such as $\mathrm{SU}(4)$. The general $\mathrm{U}(1)^{3}$ mode $^{15}$ could then be realized by Higgsing a non-Abelian theory with gauge group $\mathrm{SU}(2)^{3} \times \mathrm{SU}(3)^{3} \times \mathrm{SU}(4)$. Of course, in many cases some of the factors would be trivial, with many rank three Abelian groups realized from Higgsings of rank three non-Abelian groups such as $\mathrm{SU}(2) \times \mathrm{SU}(2) \times \mathrm{SU}(2), \mathrm{SU}(2) \times \mathrm{SU}(3)$, and $\mathrm{SU}(4)$. Degenerate divisors $A_{i}$, $B_{i j}$ and $C$ could also lead to larger product groups in an unHiggsing. As in the case of one or two $\mathrm{U}(1)$ factors, the presence of additional non-Abelian gauge groups in the original theory having U(1) factors, and/or additional geometrical structure in the base could in some situations make the unHiggsed non-Abelian theory singular, or bring the theory to a superconformal fixed point where a geometric transition would be necessary to give a smooth Calabi-Yau.

It is straightforward to generalize this kind of construction to an arbitrary number of $\mathrm{U}(1)$ factors. A very general class of $\mathrm{U}(1)^{k}$ models could then be constructed from the Higgsing of a non-Abelian theory with a gauge group $\mathrm{SU}(2)^{k} \times \mathrm{SU}(3)^{k(k-1) / 2} \times \cdots \times$ $\mathrm{SU}(k)^{k} \times \mathrm{SU}(k+1)=\prod_{i=1}^{k} \mathrm{SU}(i+1)^{c_{i}}$, where the combinatoric factor is $c_{i}=\left(\begin{array}{l}k \\ i\end{array}\right)$. For larger ranks, we could also have other non-Abelian groups arising in the unHiggsing. For rank 2, we did not need to consider $G_{2}$ separately, since it can be Higgsed to $\mathrm{SU}(3)$ on the adjoint without reducing the rank. As the rank increases, however, we can realize U(1) factors by Higgsing more general groups including $\mathrm{SO}(N)$ factors, exceptional groups such as $E_{6}, E_{7}, E_{8}$, etc. This will give rise to a rich collection of possibilities for higher rank Abelian groups that can be realized from Higgsing higher rank non-Abelian structures.

While this type of unHiggsing construction might be expected to give the most general classes of models with any number of Abelian factors there are a number of issues that would need to be addressed. First, while this story seems likely to hold in the low-energy supergravity theory, the realization in F-theory through an explicit Weierstrass model is quite nontrivial. Even for two U(1) factors, the general model with explicit formulae given in appendix A are quite complex, and do not follow in any simple way even though we can explicitly construct Weierstrass models in principle for the unHiggsed $\mathrm{SU}(2) \times \mathrm{SU}(2) \times \mathrm{SU}(3)$

\footnotetext{
${ }^{15}$ For a special construction of F-theory compactifications with $\mathrm{U}(1)^{3}$ see [13].
} 
model more directly. While as discussed in $[4,14]$, we have an explicit formulation of the Higgsing of a single $\mathrm{SU}(2)$ factor in terms of Weierstrass models, and as discussed here this can be extended to theories with multiple non-Abelian factors, this construction becomes progressively more complicated as the number of factors increases. Another issue that would need to be addressed for any complete understanding of models with more Abelian factors is the analysis of special cases where the horizontal divisors cannot be unHiggsed to vertical divisors of a non-Abelian theory with the same rank. Given that some examples of this are already encountered for two U(1) factors, we expect an even more exotic set of special cases when the number of $\mathrm{U}(1)$ factors is increased. Despite these technical issues, however, it seems that exploring the set of $\mathrm{U}(1)^{3}, \mathrm{U}(1)^{4}, \ldots$ models that can be realized in F-theory through Higgsings of rank $k$ and higher non-Abelian groups holds promise as a way of gaining some systematic understanding of these rather difficult theories.

\subsection{Exotic matter representations}

Another issue that has arisen in this paper, in which we have likely only touched on the tip of a rather complex iceberg of possibilities, is the appearance of non-standard matter representations. As discussed in section 2, an $\mathrm{SU}(2)$ or $\mathrm{SU}(3)$ factor that is tuned in Ftheory on a smooth divisor $D$ will carry generically only matter in the fundamental and adjoint representations. In $6 \mathrm{D}$ models, the number of adjoints simply corresponds to the genus $g$ of the curve $D$. As discussed in [42,43], however, when the divisor $D$ is itself singular, other representations of the gauge group can arise. A simple formula was derived in [41] from anomaly cancellation, which determines the contribution to the arithmetic genus of a curve $D$ that should be associated with the singularity carrying any given matter representation. For the adjoint and symmetric representations, the contribution is 1. Thus, in particular, as was originally suggested by Sadov in [43] (see also [61]), we expect that tuning an $\mathrm{SU}(3)$ on certain divisors with double point type singularities should give rise to matter in the symmetric representation. Indeed, a large class of the models we considered here can be associated with the Higgsing of $\mathrm{SU}(2) \times \mathrm{SU}(2) \times \mathrm{SU}(3)$ non-Abelian theories, where the $\mathrm{SU}(3)$ lies on a curve of the form

$$
t=A x^{2}+B x y+C y^{2}
$$

where there are double point singularities at the points $x=y=0$. In the simplest case, a $6 \mathrm{D}$ model on $\mathbb{P}^{2}$ with $x, y$ defining lines and $A, B, C$ defining cubics, there is a single double point singularity at $x=0$. This corresponds to a symmetric representation of $\mathrm{SU}(3)$, which carries charge $(2,2)$ in the Higgsed $\mathrm{U}(1) \times \mathrm{U}(1)$ theory. As we have discussed in earlier sections, this double point singularity apparently cannot be removed without changing the gauge group of the theory.

This is one of the first situations where we have an explicit example of a non-generic matter representation arising on a singular curve. At an algebraic level, Weierstrass models with this kind of structure are rather subtle. This can be illustrated clearly in a simple example. Consider tuning an $\mathrm{SU}(N)$ on a generic divisor $\sigma(x, y)$, where $x, y$ are coordinates on the base. As analyzed in detail in [42], this can be done systematically by tuning the 
coefficients in an expansion $f=f_{0}+f_{1} \sigma+f_{2} \sigma^{2}+\cdots, g=g_{0}+g_{1} \sigma+g_{2} \sigma^{2}+\cdots$, where $f_{0}=-3 u(x, y)^{2}, g_{0}=2 u(x, y)^{3}$, and similar conditions hold at higher orders so that the discriminant cancels at leading orders in $\sigma$, with e.g., $4 f_{0}^{3}=-108 u^{6}=-27 g_{0}^{2}$. If $\sigma$ is a singular curve, however, such as for example $\sigma=4 x^{3}+27 y^{2}$, we can have $f_{0}=x, g_{0}=y$, and the discriminant will vanish to leading order. This is the kind of condition that is automatically satisfied by the form (7.2) in the context we have encountered it here. An outstanding challenge for F-theory is to develop tools and understanding for systematically constructing such singular Weierstrass models to realize arbitrary gauge groups and matter representations that are allowed by low-energy supergravity consistency conditions such as anomaly cancellation.

Within the context of the models developed here, one question is whether there is a construction for models with $\mathrm{U}(1)$ charges associated with arbitrary numbers of symmetric representations of $\mathrm{SU}(3)$. In particular, considered over $\mathbb{P}^{2}$, the singular curve (7.2) must be of degree at least five. It should be possible, however, to construct F-theory models with an $\mathrm{SU}(3)$ on a singular quartic over $\mathbb{P}^{2}$. The quartic has genus three, so we should be able to have one or two symmetric representations, and then Higgs on the adjoint representation leaving one or two charge $(2,2)$ states in the low-energy $\mathrm{U}(1) \times \mathrm{U}(1)$ model.

More generally, we might hope to construct models with higher $\mathrm{U}(1)$ charges from more exotic representations. Even for models with a single U(1), we expect that there are solutions with matter having charge $q=3$, see [32] for the Abelian model, associated with Higgsing of an SU(2) having matter in the three-index symmetric representation. At this time no explicit geometric F-theory realization of such a matter representation is yet known. Even more exotic matter representations can in principle arise when we consider higher-rank groups.

\subsection{Matching 6D supergravity}

It was conjectured in [62] that every low-energy 6D supergravity theory can either be realized in string theory or suffers from some quantum inconsistency. The close connection between the structure of $6 \mathrm{D}$ supergravity and F-theory [40, 63] suggests that F-theory may provide a framework for systematically constructing vacua in each branch of the complete moduli space of $6 \mathrm{D}$ supergravity theories, with different branches associated with different bases for the F-theory compactification connected through tensionless string transitions. At this point, however, "string universality" for $6 \mathrm{D}$ supergravity theories has not been proven. F-theory geometry places certain additional constraints on low-energy physics. While some such constraints have been understood as consistency conditions that must be satisfied by the low-energy supergravity theories [64], other such constraints are not yet understood from the low-energy point of view. Theories with Abelian factors provide an important regime for testing the correspondence between the set of F-theory constructions and consistent supergravity theories, in which there are many open questions. Some analysis of the set of consistent solutions to the $6 \mathrm{D}$ anomaly equations in the presence of Abelian factors was carried out in $[36,37]$. In the context of the work in this paper, the question is whether a correspondence can be found between the set of apparently consistent $6 \mathrm{D}$ supergravity theories with two Abelian factors and the set of F-theory constructions. 
Two specific examples of situations where this kind of question arise have been encountered in this paper. A first question is whether the general model we have described here in fact captures all F-theory constructions with two U(1) factors. As we have noted, the $d P_{2}$ $\mathrm{U}(1) \times \mathrm{U}(1)$ models identified in [8-12] give rise to different charge spectra from the generic class of models we have constructed here, though these models are also described in the framework given here by relaxing the constraints on the divisor classes associated with the relevant parameters. It would be interesting to study whether there are other exceptional cases such as this, some of which may go beyond the construction presented here.

Another significant class of examples relates to the exotic matter configurations described in the previous section. Considering even just symmetric matter representations of $\mathrm{SU}(3)$, there are a variety of $\mathrm{U}(1) \times \mathrm{U}(1)$ models that would seem natural from the lowenergy point of view that are not reproduced by our general class of constructions. In particular, since under anomaly cancellation an adjoint behaves identically to a combination of asymmetric and a fundamental representation of $\mathrm{SU}(3)$, we can consider a general model where $\mathrm{SU}(3)$ is realized on a curve of genus $g$, and replace anywhere from 1 to $g-1$ of the generic adjoint representations with a symmetric + fundamental combination of representations. Only a small subset of these are realized through the model constructed here. For example, as mentioned above we could tune an $\mathrm{SU}(3)$ gauge group on the base $\mathbb{P}^{2}$ on a curve $C$ of degree 4 , which has genus 3 . According to low-energy considerations only, it seems that it should be possible to replace one or two adjoint representations with a symmetric + fundamental, and Higgs on the remaining adjoint, which would give a $\mathrm{U}(1) \times \mathrm{U}(1)$ model with one or two charged matter fields in the $(2,2)+(-2,0)+(0,-2)$ representations. This is impossible to realize, however, in the spectrum described in table 2. Logically there are several possibilities. It may be that these theories are actually inconsistent due to asyet not understood compatibility conditions between low-energy field theory and quantum gravity. It may be that these theories are consistent but cannot be realized in F-theory. It may be that these theories can be realized in F-theory but in a very different way than using the construction of this paper. Determining which of these possibilities is correct may be a fruitful way of uncovering further the beautiful and subtle correspondence between geometry and physics that F-theory provides.

\section{Conclusions}

We conclude by summarizing the key results of the paper:

- We have given what we believe is the most general construction of a globally defined F-theory compactication with $\mathrm{U}(1) \times \mathrm{U}(1)$ gauge factors. Specifically, we have constructed a general class of elliptically fibered Calabi-Yau manifolds with rank two Mordell-Weil group. The elliptic fibration is based on the elliptic curve $\mathcal{E}$ with three (non-toric) rational points at general positions, which is realized as a specialized cubic in $\mathbb{P}^{2}$. We have obtained the Weierstrass form for this general class of models. Though the focus is on Calabi-Yau threefolds, the construction is general and is valid for an arbitrary F-theory base manifold. The work is a generalization of the earlier 
constructions $[8-12,32]$ where the rational points in in $\mathcal{E}$ were chosen at specific positions and assumed to be toric points in $\mathbb{P}^{2}$.

- We have provided a detailed analysis of the codimension two singularities of these elliptically fibered Calabi-Yau manifolds, and have provided their global resolution, which is represented as a complete intersection in an $n+4$-dimensional ambient space, constructed by two blow-ups at two non-toric points in the ambient space of the elliptic fiber. This allows for the full determination of charges and multiplicities for the matter spectrum of general global $6 \mathrm{D} U(1) \times \mathrm{U}(1)$ F-theory models. We also explicitly checked that the matter spectra cancel all $6 \mathrm{D}$ anomalies.

The matter spectra of the models constructed here are more general and contain additional matter representations, compared to those of specialized constructions [8$12,32]$. In particular, they include representations with $(-2,-2),(-2,1)$ and $(2,0)$ charges under $\mathrm{U}(1) \times \mathrm{U}(1)$. Representations with higher charges could emerge in special cases where the elliptic curve is further specialized.

- We have provided a detailed geometric and field theoretical study of the general unHiggsing mechanism in the moduli space of globally defined F-theory models with $\mathrm{U}(1) \times \mathrm{U}(1)$ symmetry. These transitions have a geometric interpretation as the transformation of a rational section to a Cartan divisor of a non-Abelian gauge symmetry; in such an unHiggsing process, the nonlocal horizontal divisors, associated with sections in the Mordell-Weil group, become local vertical divisors, associated with the Cartan generators of non-Abelian gauge groups of Kodaira singularities in the elliptic fibration over the base. One of the principal results is that these models can be unHiggsed to a non-Abelian gauge group $\mathrm{SU}(2) \times \mathrm{SU}(2) \times \mathrm{SU}(3)$. The structure of the non-Abelian group can be understood geometrically by identifying the general form of the vertical divisors, associated with unHiggsing the two $\mathrm{U}(1)$ factors, to be $A C$ and $B C$, where $C$ is a common factor. The unHiggsing process leads to $\mathrm{SU}(2)$ factors on $A$ and $B$, while on $C$ the two elements of the Cartan generators combine, resulting in $\mathrm{SU}(3)$. We provide the geometric and the field theory analysis of the non-Abelian matter spectra that after Higgsing result in the spectra of general $\mathrm{U}(1) \times \mathrm{U}(1)$ models. In particular, we note that the appearance of matter with $(-2,-2)$ charges originates from the symmetric representation of $\mathrm{SU}(3)$.

We also note that in some cases the divisors $A, B$, and $C$ can be reducible; for example $A$ can support $\mathrm{SU}(2) \times \mathrm{SU}(2)$ or more $\mathrm{SU}(2)$ factors. In the most generic such case, the unHiggsed symmetry results in $\mathrm{SU}(2)^{3} \times \mathrm{SU}(3)$. In these cases the Higgsing to a general $\mathrm{U}(1) \times \mathrm{U}(1)$ is due to bi-fundamental non-Abelian matter only.

- An important new result that has emerged from the geometric analysis of these models and the associated unHiggsing mechanism is the first explicit geometric construction of a symmetric representation of the unitary gauge symmetry $\mathrm{SU}(N)$ for $N>2$, in this case $\mathrm{SU}(3)$. The associated Weierstrass models have an intricate and nontrivial algebraic structure. Specifically, the appearance of a symmetric representation is 
associated with the tuning of an $\mathrm{SU}(3)$ divisor with a double point type singularity that cannot be removed while retaining the $I_{3}$ Kodaira singularity type. It is expected that tuning of more intricate singular divisors supporting non-Abelian groups could result in other higher-dimensional representations, such as three-index symmetric tensor representations. These higher-dimensional representations are expected to play a role in the unHiggsing of Abelian models with larger Abelian charges.

- The $\mathrm{U}(1) \times \mathrm{U}(1)$ construction suggests a generalization to theories with any number of $\mathrm{U}(1)$ factors; for a model with $\mathrm{U}(1)^{k}$ factors with $k>2$, we expect that an unHiggsing of $k$-horizontal divisors into vertical divisors would result in an unHiggsed phase with a gauge group like $\prod_{i=1}^{k} \mathrm{SU}(i+1)^{c_{i}}$, where $c_{i}=\left(\begin{array}{c}k \\ i\end{array}\right)$.

\section{Acknowledgments}

We would like to thank Kang Sin Choi, Antonella Grassi, Thomas Grimm, James Halverson, Andreas Kapfer, Albrecht Klemm, Sven Krippendorf, Craig Lawrie, Wolfgang Lerche, David Morrison, Daniel Park, Maximilian Poretschkin, Nikhil Raghuram, Soo-Jong Rey, Sakura Schäfer-Nameki and Peng Song for helpful discussions and comments. MC would like to thank the theory group at CERN and DK the EWHA Womans University for hospitality during the final stages of this project. The work is supported in part by the DOE through (HEP) Award DE-SC0013528 (MC,HP) and (HEP) Award DE-SC00012567 (WT), in part by the Fay R. and Eugene L. Langberg Endowed Chair (MC), and in part by the Slovenian Research Agency (ARRS) (MC).

\section{A The Weierstrass form of $\boldsymbol{X}_{n+1}$}

The Weierstrass form of the elliptic curve (3.4) can be computed by first mapping to the cubic in $d P_{2}$ and then using the results of $[8,9]$ for its Weierstrass form. Alternatively, one can use Deligné's approach of constructing the Weierstrass coordinates $[z: x: y]$ as sections of appropriate line bundles on $\mathcal{E}$. Either way, we find the following expressions for $f$ and $g$ :

$$
\begin{aligned}
f= & -\frac{1}{48}\left(s_{6}^{2}-4 s_{3} s_{8}\right)^{2}+\frac{1}{2} b_{1} b_{2} b_{3} s_{3}\left(2 s_{3} s_{5}-s_{2} s_{6}\right)+\frac{1}{6}\left(a_{1} b_{2} b_{3}+a_{2} b_{1} b_{3}+a_{3} b_{1} b_{2}\right)\left(s_{2} s_{6}^{2}+s_{3}\left(2 s_{2} s_{8}-3 s_{5} s_{6}\right)\right) \\
& -\frac{1}{3}\left(a_{3}^{2} b_{1}^{2} b_{2}^{2}+a_{2}^{2} b_{1}^{2} b_{3}^{2}+a_{1}^{2} b_{2}^{2} b_{3}^{2}\right)\left(s_{2}^{2}-3 s_{1} s_{3}\right)+\frac{1}{6}\left(b_{1} a_{2} a_{3}+b_{2} a_{1} a_{3}+b_{3} a_{1} a_{2}\right)\left(2 b_{1} b_{2} b_{3}\left(s_{2}^{2}-3 s_{1} s_{3}\right)-3 s_{2} s_{6} s_{8}\right. \\
& \left.+s_{5}\left(s_{6}^{2}+2 s_{3} s_{8}\right)\right)+\frac{1}{6}\left(a_{2} a_{3}^{2} b_{1}^{2} b_{2}+a_{2}^{2} a_{3} b_{1}^{2} b_{3}+a_{1} a_{3}^{2} b_{1} b_{2}^{2}+a_{1}^{2} a_{3} b_{2}^{2} b_{3}+a_{1} a_{2}^{2} b_{1} b_{3}^{2}+a_{1}^{2} a_{2} b_{2} b_{3}^{2}\right)\left(2 s_{2} s_{5}-3 s_{1} s_{6}\right) \\
& -\frac{1}{3}\left(a_{2}^{2} a_{3}^{2} b_{1}^{2}+a_{1}^{2} a_{2}^{2} b_{3}^{2}+b_{2}^{2} a_{1}^{2} a_{3}^{2}-a_{1} a_{2} a_{3}^{2} b_{1} b_{2}-a_{1} a_{2}^{2} a_{3} b_{1} b_{3}-a_{1}^{2} a_{2} a_{3} b_{2} b_{3}\right)\left(s_{5}^{2}-3 s_{1} s_{8}\right) \\
& +a_{1} a_{2} a_{3}\left(b_{1} b_{2} b_{3}\left(3 s_{1} s_{6}-2 s_{2} s_{5}\right)+s_{2} s_{8}^{2}-\frac{1}{2} s_{5} s_{6} s_{8}\right) \\
g= & \frac{1}{864}\left(s_{6}^{2}-4 s_{3} s_{8}\right)^{3}+\frac{1}{24} b_{1} b_{2} b_{3} s_{3}\left(\left(s_{2} s_{6}-2 s_{3} s_{5}\right)\left(s_{6}^{2}-4 s_{3} s_{8}\right)+6 b_{1} b_{2} b_{3} s_{3}\left(s_{2}^{2}-4 s_{1} s_{3}\right)\right) \\
& -\frac{1}{72}\left(b_{2} b_{3} a_{1}+b_{3} b_{1} a_{2}+b_{1} b_{2} a_{3}\right)\left(12 b_{1} b_{2} b_{3} s_{3}\left(s_{2} s_{3} s_{5}+\left(s_{2}^{2}-6 s_{1} s_{3}\right) s_{6}\right)\right. \\
& \left.\quad+\left(s_{6}^{2}-4 s_{3} s_{8}\right)\left(s_{2} s_{6}^{2}+s_{3}\left(2 s_{2} s_{8}-3 s_{5} s_{6}\right)\right)\right) \\
& +\frac{1}{36}\left(b_{1}^{2} b_{2}^{2} a_{3}^{2}+b_{1}^{2} b_{3}^{2} a_{2}^{2}+b_{2}^{2} b_{3}^{2} a_{1}^{2}\right)\left(3\left(3 s_{5}^{2}-8 s_{1} s_{8}\right) s_{3}^{2}+\left(4 s_{2}^{2} s_{8}-3 s_{6}\left(2 s_{2} s_{5}+s_{1} s_{6}\right)\right) s_{3}+2 s_{2}^{2} s_{6}^{2}\right)
\end{aligned}
$$


$+\frac{1}{72}\left(b_{1} a_{2} a_{3}+b_{2} a_{1} a_{3}+b_{3} a_{1} a_{2}\right)\left(2 b_{1} b_{2} b_{3}\left(-6\left(s_{5}^{2}+2 s_{1} s_{8}\right) s_{3}^{2}+\left(2 s_{8} s_{2}^{2}+18 s_{5} s_{6} s_{2}-33 s_{1} s_{6}^{2}\right) s_{3}+s_{2}^{2} s_{6}^{2}\right)\right.$

$\left.-\left(s_{6}^{2}-4 s_{3} s_{8}\right)\left(s_{5}\left(s_{6}^{2}+2 s_{3} s_{8}\right)-3 s_{2} s_{6} s_{8}\right)\right)+\frac{1}{27}\left(b_{1}^{3} b_{2}^{3} a_{3}^{3}+b_{1}^{3} b_{3}^{3} a_{2}^{3}+b_{2}^{3} b_{3}^{3} a_{1}^{3}\right) s_{2}\left(9 s_{1} s_{3}-2 s_{2}^{2}\right)$

$+\frac{1}{72}\left(b_{1}^{2} b_{2} a_{2} a_{3}^{2}+b_{1}^{2} b_{3} a_{2}^{2} a_{3}+a_{1} a_{2}^{2} b_{1} b_{3}^{2}+a_{1}^{2} a_{2} b_{2} b_{3}^{2}+a_{1}^{2} a_{3} b_{2}^{2} b_{3}+a_{1} a_{3}^{2} b_{1} b_{2}^{2}\right)\left(4 b_{1} b_{2} b_{3} s_{2}\left(2 s_{2}^{2}-9 s_{1} s_{3}\right)\right.$

$\left.+s_{6}\left(s_{6}\left(2 s_{2} s_{5}+3 s_{1} s_{6}\right)-12 s_{3} s_{5}^{2}\right)+4\left(s_{2} s_{3} s_{5}-3\left(s_{2}^{2}-5 s_{1} s_{3}\right) s_{6}\right) s_{8}\right)$

$+\frac{1}{72} a_{1} a_{2} a_{3}\left(16 b_{1}^{2} b_{2}^{2} b_{3}^{2} s_{2}\left(9 s_{1} s_{3}-2 s_{2}^{2}\right)+6 b_{1} b_{2} b_{3}\left(s_{6}\left(6 s_{3} s_{5}^{2}+s_{6}\left(9 s_{1} s_{6}-8 s_{2} s_{5}\right)\right)\right.\right.$

$$
\left.\left.+2\left(3\left(s_{2}^{2}+2 s_{1} s_{3}\right) s_{6}-8 s_{2} s_{3} s_{5}\right) s_{8}\right)+3 s_{8}\left(s_{5} s_{6}-2 s_{2} s_{8}\right)\left(s_{6}^{2}-4 s_{3} s_{8}\right)\right)
$$

$+\frac{1}{18}\left(a_{2}^{3} a_{3} b_{1}^{3} b_{3}^{2}+a_{3}^{3} a_{2} b_{1}^{3} b_{2}^{2}+a_{3}^{3} a_{1} b_{1}^{2} b_{2}^{3}+a_{1}^{3} a_{3} b_{2}^{3} b_{3}^{2}+a_{1}^{3} a_{2} b_{2}^{2} b_{3}^{3}+a_{2}^{3} a_{1} b_{1}^{2} b_{3}^{3}\right)\left(\left(2 s_{2}^{2}-3 s_{1} s_{3}\right) s_{5}-3 s_{1} s_{2} s_{6}\right)$

$+\frac{1}{36}\left(b_{1}^{2} a_{2}^{2} a_{3}^{2}+b_{2}^{2} a_{1}^{2} a_{3}^{2}+b_{3}^{2} a_{1}^{2} a_{2}^{2}\right)\left(8 b_{1} b_{2} b_{3}\left(-2 s_{5} s_{2}^{2}+3 s_{1} s_{6} s_{2}+3 s_{1} s_{3} s_{5}\right)+2\left(s_{6}^{2}+2 s_{3} s_{8}\right) s_{5}^{2}-6 s_{2} s_{6} s_{8} s_{5}\right.$

$$
\left.-3 s_{8}\left(s_{1}\left(s_{6}^{2}+8 s_{3} s_{8}\right)-3 s_{2}^{2} s_{8}\right)\right)
$$

$+\frac{1}{36}\left(a_{1}^{2} a_{2} a_{3} b_{2} b_{3}+a_{2}^{2} a_{1} a_{3} b_{1} b_{3}+a_{3}^{2} a_{1} a_{2} b_{1} b_{2}\right)\left(4 b_{1} b_{2} b_{3}\left(\left(2 s_{2}^{2}-3 s_{1} s_{3}\right) s_{5}-3 s_{1} s_{2} s_{6}\right)\right.$

$\left.+\left(s_{6}^{2}+2 s_{3} s_{8}\right) s_{5}^{2}+18 s_{2} s_{6} s_{8} s_{5}-6\left(s_{2}^{2}+2 s_{1} s_{3}\right) s_{8}^{2}-33 s_{1} s_{6}^{2} s_{8}\right)$

$+\frac{1}{18}\left(b_{2} b_{3}^{3} a_{1}^{3} a_{2}^{2}+b_{1} b_{3}^{3} a_{2}^{3} a_{1}^{2}+b_{2}^{3} b_{3} a_{1}^{3} a_{3}^{2}+b_{1} b_{2}^{3} a_{3}^{3} a_{1}^{2}+b_{1}^{3} b_{3} a_{2}^{3} a_{3}^{2}+b_{1}^{3} b_{2} a_{3}^{3} a_{2}^{2}\right)\left(s_{2}\left(2 s_{5}^{2}-3 s_{1} s_{8}\right)-3 s_{1} s_{5} s_{6}\right)$

$+\frac{2}{9}\left(b_{1}^{2} b_{2}^{2} a_{1} a_{2} a_{3}^{3}+b_{1}^{2} b_{3}^{2} a_{1} a_{3} a_{2}^{3}+b_{2}^{2} b_{3}^{2} a_{2} a_{3} a_{1}^{3}\right)\left(3 s_{1} s_{5} s_{6}+s_{2}\left(3 s_{1} s_{8}-2 s_{5}^{2}\right)\right)$

$+\frac{1}{18}\left(b_{1} a_{1} a_{2}^{2} a_{3}^{2}+b_{2} a_{2} a_{1}^{2} a_{3}^{2}+b_{3} a_{3} a_{1}^{2} a_{2}^{2}\right)\left(2 b_{1} b_{2} b_{3}\left(s_{2}\left(2 s_{5}^{2}-3 s_{1} s_{8}\right)-3 s_{1} s_{5} s_{6}\right)\right.$

$$
\left.-3 s_{8}\left(s_{6} s_{5}^{2}+\left(s_{2} s_{5}-6 s_{1} s_{6}\right) s_{8}\right)\right)
$$

$+\frac{1}{4}\left(b_{1}^{4} b_{2}^{2} a_{2}^{2} a_{3}^{4}+b_{1}^{4} b_{3}^{2} a_{2}^{4} a_{3}^{2}+b_{2}^{2} b_{3}^{4} a_{1}^{4} a_{2}^{2}+b_{1}^{2} b_{3}^{4} a_{2}^{4} a_{1}^{2}+b_{2}^{4} b_{3}^{2} a_{1}^{4} a_{3}^{2}+b_{1}^{2} b_{2}^{4} a_{3}^{4} a_{1}^{2}\right) s_{1}^{2}$

$-\frac{1}{2}\left(b_{1}^{3} b_{2}^{3} a_{1} a_{2} a_{3}^{4}+b_{2}^{3} b_{3}^{3} a_{2} a_{3} a_{1}^{4}+b_{1}^{3} b_{3}^{3} a_{1} a_{3} a_{2}^{4}\right) s_{1}^{2}$

$-\frac{1}{54}\left(a_{1}^{3} a_{2}^{3} b_{3}^{3}+a_{2}^{3} a_{3}^{3} b_{1}^{3}+a_{1}^{3} a_{3}^{3} b_{2}^{3}\right)\left(9 s_{1}\left(3 b_{1} b_{2} b_{3} s_{1}-2 s_{5} s_{8}\right)+4 s_{5}^{3}\right)$

$+\frac{1}{18}\left(b_{1}^{2} b_{2} a_{1} a_{2}^{2} a_{3}^{3}+b_{1} b_{2}^{2} a_{2} a_{1}^{2} a_{3}^{3}+b_{1}^{2} b_{3} a_{1} a_{3}^{2} a_{2}^{3}+b_{3} b_{1}^{2} a_{1} a_{3}^{2} a_{2}^{3}+b_{2}^{2} b_{3} a_{2} a_{3}^{2} a_{1}^{3}\right.$

$$
\left.+b_{2} b_{3}^{2} a_{3} a_{2}^{2} a_{1}^{3}\right)\left(9 b_{1} b_{2} b_{3} s_{1}^{2}+2 s_{5}^{3}-9 s_{1} s_{8} s_{5}\right)
$$

$+\left(a_{1} a_{2} a_{3}\right)^{2}\left(-\frac{3}{2} b_{1}^{2} b_{2}^{2} b_{3}^{2} s_{1}^{2}+\frac{2}{9} b_{1} b_{2} b_{3} s_{5}\left(9 s_{1} s_{8}-2 s_{5}^{2}\right)+\frac{1}{4} s_{8}^{2}\left(s_{5}^{2}-4 s_{1} s_{8}\right)\right)$.

We can use Weierstrass coordinates of the rational points of the elliptic curve in $d P_{2}$ in [9] to obtain the Weierstrass coordinates of the rational points (3.5). This yields

$$
\begin{aligned}
z_{Q}= & a_{1} b_{2}-a_{2} b_{1} \\
x_{Q}= & b_{1}^{2} b_{2}^{2} s_{3}^{2}-b_{1} b_{2}\left(a_{2} b_{1}+a_{1} b_{2}\right) s_{3} s_{6}+\frac{1}{12}\left(a_{2}^{2} b_{1}^{2}+a_{1}^{2} b_{2}^{2}\right)\left(s_{6}^{2}+8 s_{3} s_{8}\right)+\frac{1}{6} a_{1} a_{2} b_{1} b_{2}\left(5 s_{6}^{2}+4 s_{3} s_{8}\right) \\
& +\frac{1}{3}\left(a_{2} b_{1}-a_{1} b_{2}\right)^{2}\left(\left(2 a_{3} b_{1} b_{2}-a_{2} b_{1} b_{3}-a_{1} b_{2} b_{3}\right) s_{2}+\left(2 a_{1} a_{2} b_{3}-a_{1} a_{3} b_{2}-a_{2} a_{3} b_{1}\right) s_{5}\right) \\
& -a_{1} a_{2}\left(a_{2} b_{1}+a_{1} b_{2}\right) s_{6} s_{8}+a_{1}^{2} a_{2}^{2} s_{8}^{2} \\
y_{Q}= & -b_{1}^{3} b_{2}^{3} s_{3}^{3}+\frac{1}{2}\left(a_{2} b_{1}-a_{1} b_{2}\right)^{4}\left(a_{3} b_{1}-a_{1} b_{3}\right)\left(a_{2} b_{3}-a_{3} b_{2}\right) s_{1}-\frac{1}{2} b_{2}^{4} b_{3} s_{3} s_{5} a_{1}^{4}-\frac{1}{2} a_{3} b_{2}^{4} s_{2} s_{8} a_{1}^{4} \\
& +\frac{1}{2} a_{2} b_{2}^{3} b_{3}\left(s_{5} s_{6}+s_{2} s_{8}\right) a_{1}^{4}+\frac{1}{2} a_{2} a_{3} b_{2}^{3} s_{5} s_{8} a_{1}^{4}-a_{2}^{2} b_{2}^{2} b_{3} s_{5} s_{8} a_{1}^{4}+\frac{1}{2} a_{3} b_{1} b_{2}^{4}\left(s_{3} s_{5}+s_{2} s_{6}\right) a_{1}^{3} \\
& +a_{2} a_{3} b_{1} b_{2}^{3}\left(s_{2} s_{8}-s_{5} s_{6}\right) a_{1}^{3}+\frac{1}{2} b_{2}^{3} s_{3}\left(b_{1} b_{2} b_{3} s_{2}+s_{6} s_{8}\right) a_{1}^{3}-\frac{1}{2} a_{2}^{2} a_{3} b_{1} b_{2}^{2} s_{5} s_{8} a_{1}^{3} \\
& +a_{2}^{3}\left(-s_{8}^{3}+2 b_{1} b_{2} b_{3} s_{5} s_{8}\right) a_{1}^{3}-\frac{1}{2} a_{2}^{2} b_{2}\left(b_{1} b_{2} b_{3}\left(s_{5} s_{6}+s_{2} s_{8}\right)-3 s_{6} s_{8}^{2}\right) a_{1}^{3} \\
& +\frac{1}{2} a_{2} b_{2}^{2}\left(2 b_{1} b_{2} b_{3}\left(s_{3} s_{5}-s_{2} s_{6}\right)-s_{8}\left(s_{6}^{2}+2 s_{3} s_{8}\right)\right) a_{1}^{3}-a_{3} b_{1}^{2} b_{2}^{4} s_{2} s_{3} a_{1}^{2}-\frac{1}{2} a_{2} a_{3} b_{1}^{2} b_{2}^{3}\left(s_{3} s_{5}+s_{2} s_{6}\right) a_{1}^{2}
\end{aligned}
$$


$-\frac{1}{2} b_{1} b_{2}^{3} s_{3}\left(s_{6}^{2}+2 s_{3} s_{8}\right) a_{1}^{2}+\frac{1}{2} a_{2} b_{1} b_{2}^{2}\left(s_{6}^{3}+5 s_{3} s_{8} s_{6}-b_{1} b_{2} b_{3} s_{2} s_{3}\right) a_{1}^{2}+a_{2}^{2} a_{3} b_{1}^{2} b_{2}^{2}\left(2 s_{5} s_{6}-s_{2} s_{8}\right) a_{1}^{2}$

$+\frac{1}{2} a_{2}^{3} a_{3} b_{1}^{2} b_{2}\left(2 b_{1} b_{2} b_{3} s_{1}-s_{5} s_{8}\right) a_{1}^{2}+a_{2}^{4} b_{1}^{2} b_{3}\left(-s_{5} s_{8}\right) a_{1}^{2}-\frac{1}{2} a_{2}^{3} b_{1}\left(b_{1} b_{2} b_{3}\left(s_{5} s_{6}+s_{2} s_{8}\right)-3 s_{6} s_{8}^{2}\right) a_{1}^{2}$

$-a_{2}^{2} b_{1} b_{2}\left(b_{1} b_{2} b_{3}\left(s_{3} s_{5}-2 s_{2} s_{6}\right)+s_{8}\left(2 s_{6}^{2}+s_{3} s_{8}\right)\right) a_{1}^{2}+2 a_{2} a_{3} b_{1}^{3} b_{2}^{3} s_{2} s_{3} a_{1}+\frac{3}{2} b_{1}^{2} b_{2}^{3} s_{3}^{2} s_{6} a_{1}$

$-\frac{1}{2} a_{2}^{2} a_{3} b_{1}^{3} b_{2}^{2}\left(s_{3} s_{5}+s_{2} s_{6}\right) a_{1}+a_{2}^{3} a_{3} b_{1}^{3} b_{2}\left(s_{2} s_{8}-s_{5} s_{6}\right) a_{1}+\frac{1}{2} a_{2}^{4} b_{1}^{3} b_{3}\left(s_{5} s_{6}+s_{2} s_{8}\right) a_{1}$

$-a_{2} b_{1}^{2} b_{2}^{2} s_{3}\left(2 s_{6}^{2}+s_{3} s_{8}\right) a_{1}+\frac{1}{2} a_{2}^{4} a_{3} b_{1}^{3}\left(s_{5} s_{8}\right) a_{1}+\frac{1}{2} a_{2}^{2} b_{1}^{2} b_{2}\left(s_{6}^{3}+5 s_{3} s_{8} s_{6}-b_{1} b_{2} b_{3} s_{2} s_{3}\right) a_{1}$

$+\frac{1}{2} a_{2}^{3} b_{1}^{2}\left(2 b_{1} b_{2} b_{3}\left(s_{3} s_{5}-s_{2} s_{6}\right)-s_{8}\left(s_{6}^{2}+2 s_{3} s_{8}\right)\right) a_{1}-\frac{1}{2} a_{2}^{4} a_{3}^{2} b_{1}^{5} b_{2} s_{1}-a_{2}^{2} a_{3} b_{1}^{4} b_{2}^{2} s_{2} s_{3}$

$-\frac{1}{2} a_{2}^{4} b_{1}^{4} b_{3} s_{3} s_{5}+\frac{3}{2} a_{2} b_{1}^{3} b_{2}^{2} s_{3}^{2} s_{6}+\frac{1}{2} a_{2}^{3} a_{3} b_{1}^{4} b_{2}\left(s_{3} s_{5}+s_{2} s_{6}\right)-\frac{1}{2} a_{2}^{4} a_{3} b_{1}^{4} s_{2} s_{8}-\frac{1}{2} a_{2}^{2} b_{1}^{3} b_{2} s_{3}\left(s_{6}^{2}+2 s_{3} s_{8}\right)$

$+\frac{1}{2} a_{2}^{3} b_{1}^{3} s_{3}\left(b_{1} b_{2} b_{3} s_{2}+s_{6} s_{8}\right)$

and, using the symmetry $\left(a_{2}, b_{2}\right) \leftrightarrow\left(a_{3}, b_{3}\right)$ of $\mathcal{E}$,

$$
\begin{aligned}
& z_{R}=a_{1} b_{3}-a_{3} b_{1}, \\
& x_{R}=b_{1}^{2} b_{3}^{2} s_{3}^{2}-b_{1} b_{3}\left(a_{3} b_{1}+a_{1} b_{3}\right) s_{3} s_{6}+\frac{1}{12}\left(a_{3}^{2} b_{1}^{2}+a_{1}^{2} b_{3}^{2}\right)\left(s_{6}^{2}+8 s_{3} s_{8}\right)+\frac{1}{6} a_{1} a_{3} b_{1} b_{3}\left(5 s_{6}^{2}+4 s_{3} s_{8}\right) \\
& +\frac{1}{3}\left(a_{3} b_{1}-a_{1} b_{3}\right)^{2}\left(\left(2 a_{2} b_{1} b_{3}-a_{3} b_{1} b_{2}-a_{1} b_{2} b_{3}\right) s_{2}+\left(2 a_{1} a_{3} b_{2}-a_{1} a_{2} b_{3}-a_{2} a_{3} b_{1}\right) s_{5}\right) \\
& -a_{1} a_{3}\left(a_{3} b_{1}+a_{1} b_{3}\right) s_{6} s_{8}+a_{1}^{2} a_{3}^{2} s_{8}^{2}, \\
& y_{Q}=-b_{1}^{3} b_{3}^{3} s_{3}^{3}+\frac{1}{2}\left(a_{3} b_{1}-a_{1} b_{3}\right)^{4}\left(a_{2} b_{1}-a_{1} b_{2}\right)\left(a_{3} b_{2}-a_{2} b_{3}\right) s_{1}-\frac{1}{2} b_{3}^{4} b_{2} s_{3} s_{5} a_{1}^{4}-\frac{1}{2} a_{2} b_{3}^{4} s_{2} s_{8} a_{1}^{4} \\
& +\frac{1}{2} a_{3} b_{3}^{3} b_{2}\left(s_{5} s_{6}+s_{2} s_{8}\right) a_{1}^{4}+\frac{1}{2} a_{2} a_{3} b_{3}^{3} s_{5} s_{8} a_{1}^{4}-a_{3}^{2} b_{3}^{2} b_{2} s_{5} s_{8} a_{1}^{4}+\frac{1}{2} a_{2} b_{1} b_{3}^{4}\left(s_{3} s_{5}+s_{2} s_{6}\right) a_{1}^{3} \\
& +a_{2} a_{3} b_{1} b_{3}^{3}\left(s_{2} s_{8}-s_{5} s_{6}\right) a_{1}^{3}+\frac{1}{2} b_{3}^{3} s_{3}\left(b_{1} b_{2} b_{3} s_{2}+s_{6} s_{8}\right) a_{1}^{3}-\frac{1}{2} a_{3}^{2} a_{2} b_{1} b_{3}^{2} s_{5} s_{8} a_{1}^{3} \\
& +a_{3}^{3}\left(-s_{8}^{3}+2 b_{1} b_{2} b_{3} s_{5} s_{8}\right) a_{1}^{3}-\frac{1}{2} a_{3}^{2} b_{3}\left(b_{1} b_{2} b_{3}\left(s_{5} s_{6}+s_{2} s_{8}\right)-3 s_{6} s_{8}^{2}\right) a_{1}^{3} \\
& +\frac{1}{2} a_{3} b_{3}^{2}\left(2 b_{1} b_{2} b_{3}\left(s_{3} s_{5}-s_{2} s_{6}\right)-s_{8}\left(s_{6}^{2}+2 s_{3} s_{8}\right)\right) a_{1}^{3}-a_{2} b_{1}^{2} b_{3}^{4} s_{2} s_{3} a_{1}^{2}-\frac{1}{2} a_{2} a_{3} b_{1}^{2} b_{3}^{3}\left(s_{3} s_{5}+s_{2} s_{6}\right) a_{1}^{2} \\
& -\frac{1}{2} b_{1} b_{3}^{3} s_{3}\left(s_{6}^{2}+2 s_{3} s_{8}\right) a_{1}^{2}+\frac{1}{2} a_{2} b_{1} b_{3}^{2}\left(s_{6}^{3}+5 s_{3} s_{8} s_{6}-b_{1} b_{2} b_{3} s_{2} s_{3}\right) a_{1}^{2}+a_{3}^{2} a_{2} b_{1}^{2} b_{3}^{2}\left(2 s_{5} s_{6}-s_{2} s_{8}\right) a_{1}^{2} \\
& +\frac{1}{2} a_{3}^{3} a_{3} b_{1}^{2} b_{3}\left(2 b_{1} b_{2} b_{3} s_{1}-s_{5} s_{8}\right) a_{1}^{2}+a_{3}^{4} b_{1}^{2} b_{2}\left(-s_{5} s_{8}\right) a_{1}^{2}-\frac{1}{2} a_{3}^{3} b_{1}\left(b_{1} b_{2} b_{3}\left(s_{5} s_{6}+s_{2} s_{8}\right)-3 s_{6} s_{8}^{2}\right) a_{1}^{2} \\
& -a_{3}^{2} b_{1} b_{3}\left(b_{1} b_{2} b_{3}\left(s_{3} s_{5}-2 s_{2} s_{6}\right)+s_{8}\left(2 s_{6}^{2}+s_{3} s_{8}\right)\right) a_{1}^{2}+2 a_{2} a_{3} b_{1}^{3} b_{3}^{3} s_{2} s_{3} a_{1}+\frac{3}{2} b_{1}^{2} b_{3}^{3} s_{3}^{2} s_{6} a_{1} \\
& -\frac{1}{2} a_{3}^{2} a_{2} b_{1}^{3} b_{3}^{2}\left(s_{3} s_{5}+s_{2} s_{6}\right) a_{1}+a_{3}^{3} a_{2} b_{1}^{3} b_{3}\left(s_{2} s_{8}-s_{5} s_{6}\right) a_{1}+\frac{1}{2} a_{3}^{4} b_{1}^{3} b_{2}\left(s_{5} s_{6}+s_{2} s_{8}\right) a_{1} \\
& -a_{3} b_{1}^{2} b_{2}^{2} s_{3}\left(2 s_{6}^{2}+s_{3} s_{8}\right) a_{1}+\frac{1}{2} a_{3}^{4} a_{2} b_{1}^{3}\left(s_{5} s_{8}\right) a_{1}+\frac{1}{2} a_{3}^{2} b_{1}^{2} b_{3}\left(s_{6}^{3}+5 s_{3} s_{8} s_{6}-b_{1} b_{2} b_{3} s_{2} s_{3}\right) a_{1} \\
& +\frac{1}{2} a_{3}^{3} b_{1}^{2}\left(2 b_{1} b_{2} b_{3}\left(s_{3} s_{5}-s_{2} s_{6}\right)-s_{8}\left(s_{6}^{2}+2 s_{3} s_{8}\right)\right) a_{1}-\frac{1}{2} a_{3}^{4} a_{2}^{2} b_{1}^{5} b_{2} s_{1}-a_{3}^{2} a_{2} b_{1}^{4} b_{2}^{2} s_{2} s_{3} \\
& -\frac{1}{2} a_{3}^{4} b_{1}^{4} b_{3} s_{3} s_{5}+\frac{3}{2} a_{3} b_{1}^{3} b_{3}^{2} s_{3}^{2} s_{6}+\frac{1}{2} a_{3}^{3} a_{2} b_{1}^{4} b_{3}\left(s_{3} s_{5}+s_{2} s_{6}\right)-\frac{1}{2} a_{3}^{4} a_{3} b_{1}^{4} s_{2} s_{8}-\frac{1}{2} a_{3}^{2} b_{1}^{3} b_{3} s_{3}\left(s_{6}^{2}+2 s_{3} s_{8}\right) \\
& +\frac{1}{2} a_{3}^{3} b_{1}^{3} s_{3}\left(b_{1} b_{2} b_{3} s_{2}+s_{6} s_{8}\right) \text {. }
\end{aligned}
$$

\section{B Weierstrass models with $I_{2}$ and $I_{3}$ singularities}

In this appendix, we summarize and study the general Weierstrass models of Calabi-Yau elliptic fibrations with $I_{2}$ and $I_{3}$ singularities (mostly the split case) at codimension one in the base $B_{n}$. These are the simplest singularities used to engineer F-theory models with 
gauge algebras $\mathrm{su}(2)$ and $\mathrm{su}(3)$. Here, we consider two models with rank two gauge algebra, namely $\mathrm{su}(2) \oplus \mathrm{su}(2)$ and $\mathrm{su}(3)$, and one model with an $\mathrm{su}(2) \oplus \mathrm{su}(2) \oplus \mathrm{su}(3)$ gauge algebra. The corresponding Weierstrass models serve as reference points for comparison with the Weierstrass models obtained in the main text by unHiggsing the Calabi-Yau manifolds $X_{n+1}$ with rank two Mordell-Weil group.

As we are interested in the most generic Weierstrass forms, i.e. those with the most complex structure moduli, we only consider $I_{n}$ singularities. Other realizations of rank two gauge algebras, such as Kodaira fibers of type $I I I$ and $I V$, can be obtained form these models via additional tunings as analyzed in Tate's algorithm, so that e.g. $f$ and $g$ vanish to higher order.

\section{B.1 The $\mathrm{SU}(2) \times \mathrm{SU}(2)$ Weierstrass model}

An F-theory model with two $\mathrm{su}(2)$ gauge algebras on two distinct divisors $\sigma=0, \tau=0$ in $B_{n}$, which we simply denote $\sigma$ and $\tau$ by abuse of notation, is specified by a Weierstrass model with two $I_{2}$ singularities. It can be constructed using Tate's algorithm yielding

$$
y^{2}=x^{3}+\left(-\frac{1}{3} \tilde{a}_{2}^{2}+\tilde{a}_{4} \sigma \tau\right) x z^{4}+\left(\frac{2}{27} \tilde{a}_{2}^{3}-\frac{1}{3} \tilde{a}_{2} \tilde{a}_{4} \sigma \tau+\tilde{a}_{6} \sigma^{2} \tau^{2}\right) z^{6},
$$

where the coefficients $\tilde{a}_{i}$ are sections of the line bundles

$$
\tilde{a}_{2} \in \mathcal{O}\left(-2 K_{B}\right), \quad \tilde{a}_{4} \in \mathcal{O}\left(-4 K_{B}-[\sigma]-[\tau]\right), \quad \tilde{a}_{6} \in \mathcal{O}\left(-6 K_{B}-2[\sigma]-2[\tau]\right)
$$

on $B_{n}$ by the requirement that $f \in \mathcal{O}\left(-4 K_{B}\right)$ and $g \in \mathcal{O}\left(-6 K_{B}\right)$. These coefficients are expressed by the leading Tate coefficients $\mathfrak{a}_{i}^{(k)}, i=1,2,3,4,6,{ }^{16}$ in a Tate form as

$$
\tilde{a}_{2}=\frac{1}{4}\left(4 \mathfrak{a}_{2}^{(0)}+\left(\mathfrak{a}_{1}^{(0)}\right)^{2}\right), \quad \tilde{a}_{4}=\mathfrak{a}_{4}^{(1)}+\frac{1}{2} \mathfrak{a}_{1}^{(0)} \mathfrak{a}_{3}^{(1)}, \quad \tilde{a}_{6}=\mathfrak{a}_{6}^{(2)}+\frac{1}{4}\left(\mathfrak{a}_{3}^{(1)}\right)^{2} .
$$

Any model with two $I_{2}$ singularities on smooth divisors can be shown to assume this form [42].

We are interested in models where both $\mathrm{SU}(2)$ 's carry adjoints. In $6 \mathrm{D}$, the number of adjoints is computed by the topological genus $g_{1}, g_{2}$ of the curves $\sigma, \tau$ which agrees with the arithmetic genus for smooth curves. The latter is computed as

$$
g_{i}=1+\frac{1}{2}[\Sigma] \cdot\left([\Sigma]+K_{B}\right), \quad i=1,2,
$$

with $\Sigma=\sigma, \tau$. Thus, demanding $g_{i} \geq 1$ implies

$$
[\sigma]=-K_{B}+Y, \quad[\tau]=-K_{B}+Z,
$$

for effective classes $Y, Z$. Thus, effectiveness of the sections in (B.2) implies upper bounds on both $Y$ and $Z$,

$$
Y+Z \leq-K_{B}
$$

for the model to be generic.

\footnotetext{
${ }^{16}$ We expand the Tate coefficients as $\mathfrak{a}_{i}=(\sigma \tau)^{k} \mathfrak{a}_{i}^{(k)}$ where $k$ is according to Tate's algorithm $k=0,0,1,1,2$ for an $I_{2}$ singularity.
} 
For a concrete base $B_{2}=\mathbb{P}^{2}$ for which $\mathcal{O}\left(-K_{B}\right)=\mathcal{O}_{\mathbb{P}^{2}}(3)$ this condition yields the following list of allowed values for the degree of the divisors $\sigma$ and $\tau$, together with the corresponding degrees of the $\tilde{a}_{i}$ :

\begin{tabular}{|c|c||c|c|c|}
\hline$[\sigma]$ & {$[\tau]$} & $\tilde{a}_{2}$ & $\tilde{a}_{4}$ & $\tilde{a}_{6}$ \\
\hline 6 & 3 & 6 & 3 & 0 \\
5 & 4 & 6 & 3 & 0 \\
5 & 3 & 6 & 4 & 2 \\
4 & 5 & 6 & 3 & 0 \\
4 & 4 & 6 & 4 & 2 \\
4 & 3 & 6 & 5 & 4 \\
3 & 6 & 6 & 3 & 0 \\
3 & 5 & 6 & 4 & 2 \\
3 & 4 & 6 & 5 & 4 \\
3 & 3 & 6 & 6 & 6 \\
\hline
\end{tabular}

The generic matter spectrum of the theory can be extracted directly from its Weierstrass model (B.1). By analyzing the orders of vanishing of $f, g$ and the discriminant

$$
\Delta=4 f^{3}+27 g^{2}=\sigma^{2} \tau^{2}\left(4 \tilde{a}_{2}^{3} \tilde{a}_{6}-\tilde{a}_{2}^{2} \tilde{a}_{4}^{2}+\sigma \tau\left(4 \tilde{a}_{4}^{3}-18 \tilde{a}_{2} \tilde{a}_{4} \tilde{a}_{6}\right)+27 \tilde{a}_{6}^{2} \sigma^{2} \tau^{2}\right),
$$

we find the following singular fibers and corresponding local (non-adjoint) matter representations:

\begin{tabular}{|c|c|c|}
\hline Representation & Multiplicity & Fiber \\
\hline$(\mathbf{2}, \mathbf{1})$ & $-2[\sigma] \cdot\left(4\left[K_{B}\right]+[\sigma]+[\tau]\right)$ & $I_{3}$ \\
\hline$(\mathbf{1}, \mathbf{2})$ & $-2[\tau] \cdot\left(4\left[K_{B}\right]+[\sigma]+[\tau]\right)$ & $I_{3}$ \\
\hline$(\mathbf{2}, \mathbf{2})$ & {$[\sigma] \cdot[\tau]$} & $I_{4}$ \\
\hline
\end{tabular}

We note that there are additional singular fibers of Kodaira type $I I I$ at the codimension two loci $\tilde{a}_{2}=0$ and $\sigma=0$ or $\tau=0$, respectively. As the number of fiber components does not increase at these loci, there is no matter supported at these loci.

We readily check using the data in (B.4), (B.9) and the anomaly coefficients $[\sigma],[\tau]$ for the two $\mathrm{SU}(2)$ groups that all $6 \mathrm{D}$ anomalies are canceled.

\section{B.2 The $I_{3}$ Weierstrass model}

The canonical ansatz for an F-theory model with su(3) gauge algebra is a Weierstrass model with an $I_{3}$-singularity at codimension one. It is well-know that one has to distinguish between the split, $I_{3}^{s}$, and non-split, $I_{3}^{n s}$, case. Given the Weierstrass form of an elliptic fibration with $I_{3}$-singularity at $t=0$ in the base $B_{n}$, the split condition is expressed as the condition that the following monodromy cover is reducible, i.e. has two distinct rational roots [60]:

$$
\Psi^{2}+\left.\frac{9 g}{2 f}\right|_{t=0}=0
$$


This is the case, if and only if $\left.\frac{9 g}{2 f}\right|_{t=0}$ is a perfect square. As shown in section 5.7 it is sufficient if this condition holds locally around $t=0$ requiring, however, a novel structure of the Weierstrass model.

The Weierstrass form in the non-split case is discussed in [65]. In this case the gauge algebra is generically $\mathrm{sp}(1)=\mathrm{su}(2)$ due to the monodromy action on the fiber around the branch points of the monodromy cover (5.37). Thus, we disregard the non-split case in the following.

In the split case the gauge algebra is $\mathrm{su}(3)$ and the Weierstrass model assumes, if $\left.\frac{9 g}{2 f}\right|_{t=0}$ is globally a square, the standard form that follows from Tate's algorithm [65]:

$$
y^{2}=x^{3}+\left(-\frac{1}{48} \tilde{a}_{1}^{4}+\frac{1}{2} \tilde{a}_{1} \tilde{a}_{3} t+\tilde{a}_{4} t^{2}\right) x z^{4}+\left(\frac{1}{864} \tilde{a}_{1}^{6}-\frac{1}{24} \tilde{a}_{3} \tilde{a}_{1}^{3} t+\frac{1}{12}\left(3 \tilde{a}_{3}^{2}-\tilde{a}_{1}^{2} \tilde{a}_{4}\right) t^{2}+\tilde{a}_{6} t^{3}\right) z^{6} .
$$

This is the form that is appropriate when $t$ is a smooth divisor, which supports adjoint but not symmetric representations of SU(3). Denoting the divisor $t=0$ supporting the su(3) by $t$ by abuse of notation, we find that the coefficients $\tilde{a}_{i}$ have to be sections of the following line bundles on $B_{n}$ :

$$
\tilde{a}_{1} \in \mathcal{O}\left(-K_{B}\right), \quad \tilde{a}_{3} \in \mathcal{O}\left(-3 K_{B}-[t]\right), \quad \tilde{a}_{4} \in \mathcal{O}\left(-4 K_{B}-2[t]\right), \quad \tilde{a}_{6} \in \mathcal{O}\left(-6 K_{B}-3[t]\right) .
$$

In terms of the leading Tate coefficients $\mathfrak{a}_{i}^{(k)}$ in a Tate model, ${ }^{17}$ they can be expressed as $\tilde{a}_{1}=\mathfrak{a}_{1}^{(0)}, \quad \tilde{a}_{3}=\frac{1}{3}\left(3 \mathfrak{a}_{3}^{(1)}-\mathfrak{a}_{1}^{(0)} \mathfrak{a}_{2}^{(1)}\right), \quad \tilde{a}_{4}=\mathfrak{a}_{4}^{(2)}-\frac{1}{3}\left(\mathfrak{a}_{2}^{(1)}\right)^{2}, \quad \tilde{a}_{6}=\mathfrak{a}_{6}^{(3)}-\frac{1}{3} \mathfrak{a}_{2}^{(1)} \mathfrak{a}_{4}^{(2)}+\frac{2}{27}\left(\mathfrak{a}_{2}^{(1)}\right)^{3}$.

In $6 \mathrm{D}$, the genus of the curve $t$ counts the number of matter fields in the adjoint representation of the $\mathrm{su}(3)$. As before, we have at least one adjoint if

$$
[t]=-K_{B}+Z
$$

for an effective divisor $Z$. Effectiveness of all coefficients in (B.12) imposes an upper bound on $Z$ of the form

$$
Z \leq-K_{B}
$$

so that the model (B.11) is generic.

For the concrete base $B=\mathbb{P}^{2}$ this condition is solved for the following choices of the degree of $[t]$, implying corresponding classes for the coefficients $\tilde{a}_{i}$ :

\begin{tabular}{|c||c|c|c|c|}
\hline$[t]$ & $\tilde{a}_{1}$ & $\tilde{a}_{3}$ & $\tilde{a}_{4}$ & $\tilde{a}_{6}$ \\
\hline 6 & 3 & 3 & 0 & 0 \\
5 & 3 & 4 & 2 & 3 \\
4 & 3 & 5 & 4 & 6 \\
3 & 3 & 6 & 6 & 9 \\
\hline
\end{tabular}

\footnotetext{
${ }^{17}$ Again we expand the Tate coefficients as $\mathfrak{a}_{i}=(t)^{k} \mathfrak{a}_{i}^{(k)}$ where $k$ is according to Tate's algorithm $k=$ $0,1,1,2,3$ for an $I_{3}^{\mathrm{s}}$-singularity.
} 
As before, we can extract the generic matter spectrum of the theory directly from its Weierstrass model (B.11). By analyzing the orders of vanishing of $f, g$ and the discriminant

$$
\Delta=4 f^{3}+27 g^{2}=t^{3}\left(\frac{1}{16} \tilde{a}_{1}^{3}\left(\tilde{a}_{1}^{3} \tilde{a}_{6}-\tilde{a}_{3}^{3}-\tilde{a}_{1}^{2} \tilde{a}_{3} \tilde{a}_{4}\right)+\mathcal{O}(t)\right)
$$

we find the following singular fibers and corresponding local matter representations:

\begin{tabular}{|c|c|c|}
\hline Representation & Multiplicity & Fiber \\
\hline $\mathbf{3}$ & $-3[t] \cdot\left(3 K_{B}+[t]\right)$ & $I_{4}$ \\
\hline
\end{tabular}

There are additional singular fibers of Kodaira type $I V$ at the codimension two loci $t=$ $\tilde{a}_{1}=0$. As the number of fiber components does not increase, there is no matter supported at these loci.

We readily check using the data in (B.18) and the anomaly coefficient $[t]$ that all $6 \mathrm{D}$ anomalies are canceled.

\section{B.3 The $\mathrm{SU}(2) \times \mathrm{SU}(2) \times \mathrm{SU}(3)$ Weierstrass model}

Finally, we combine the previous two subsections to construct the Weierstrass model of an elliptic fibration with two $I_{2}$ and one $I_{3}^{s}$ singularity. The Weierstrass form we obtain agrees precisely with (5.25) of the unHiggsed specialized model $X_{n+1}$.

All we have to do is to combine the forms (B.1) and (B.11), where the appropriate way to specialize coefficients is determined by Tate's algorithm. For example, we can start with the Weierstrass form (B.1) in the parametrization (B.3) for two $I_{2}$ singularities and impose the orders of vanishing $(0,1,1,2,3)$ of the Tate coefficients $\mathfrak{a}_{i}$ on the $\mathrm{SU}(3)$ divisor $t=0$ for an $I_{3}^{s}$ singularity [58]. We expand the Tate coefficients as $\mathfrak{a}_{i}=\mathfrak{a}_{i}^{(k, l)} t^{k}(\sigma \tau)^{l}$. Suppressing the superscripts on the $\mathfrak{a}_{i}^{(k, l)}$ to unclutter our notation we thus obtain the Weierstrass form appropriate for smooth $\sigma, \tau, t$

$$
\begin{aligned}
y^{2}= & x^{3}+\left(-\frac{1}{48}\left(4 \mathfrak{a}_{2} t+\mathfrak{a}_{1}^{2}\right)^{2}+\left(\mathfrak{a}_{4} t+\frac{1}{2} \mathfrak{a}_{1} \mathfrak{a}_{3}\right) \sigma \tau t\right) x z^{4} \\
& +\left(\frac{1}{864}\left(4 \mathfrak{a}_{2} t+\mathfrak{a}_{1}^{2}\right)^{3}-\frac{1}{12}\left(4 \mathfrak{a}_{2} t+\mathfrak{a}_{1}^{2}\right)\left(\mathfrak{a}_{4} t+\frac{1}{2} \mathfrak{a}_{1} \mathfrak{a}_{3}\right) \sigma \tau t+\left(\mathfrak{a}_{6} t+\frac{1}{4} \mathfrak{a}_{3}^{2}\right) \sigma^{2} \tau^{2} t^{2}\right) z^{6}
\end{aligned}
$$

where the leading Tate coefficients $\mathfrak{a}_{i}$ are sections of the line bundles

$$
\begin{array}{ll}
\mathfrak{a}_{1} \in \mathcal{O}\left(-K_{B}\right), & \mathfrak{a}_{2} \in \mathcal{O}\left(-2 K_{B}-[t]\right), \\
\mathfrak{a}_{3} \in \mathcal{O}\left(-3 K_{B}-[\sigma]-[\tau]-[t]\right), & \mathfrak{a}_{4} \in \mathcal{O}\left(-4 K_{B}-[\sigma]-[\tau]-2[t]\right), \\
\mathfrak{a}_{6} \in \mathcal{O}\left(-6 K_{B}-2[\sigma]-2[\tau]-3[t]\right) . &
\end{array}
$$

Since we have analyzed the case of $\mathrm{SU}(2) \times \mathrm{SU}(2)$ or $\mathrm{SU}(3)$ gauge groups already in the previous two subsections, we focus here only on the cases where $[t]$ is a non-trivial class and not both $[\sigma]$ and $[\tau]$ are trivial simultaneously. (We thus include not only models with gauge group $\mathrm{SU}(2) \times \mathrm{SU}(2) \times \mathrm{SU}(3)$, but also with $\mathrm{SU}(2) \times \mathrm{SU}(3))$. For the concrete base 
$B_{2}=\mathbb{P}^{2}$ for which $\mathcal{O}\left(-K_{B}\right)=\mathcal{O}_{\mathbb{P}^{2}}(3)$ the effectiveness conditions implied by (B.20) yield the following list of allowed values for the degrees of the divisors $[\sigma],[\tau]$ and $[t]$ :

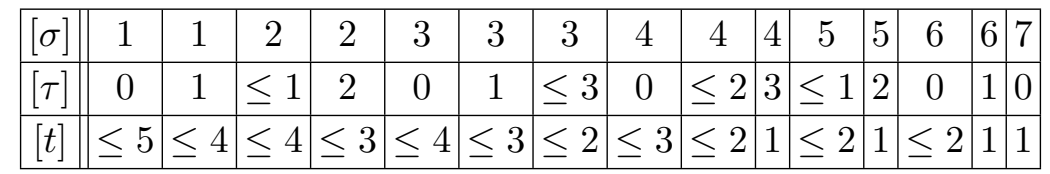

Here we indicate by $\leq k$ that all integers less or equal to $k$ are allowed. In addition, we obtain valid models by exchanging the degrees of $[\sigma]$ and $[\tau]$.

The generic local matter spectrum of the theory can be extracted directly from its Weierstrass model (5.22). By analyzing the orders of vanishing of $f, g$ and the discriminant, we find the following singular fibers and corresponding matter representations:

\begin{tabular}{|c|c|}
\hline Representation & Multiplicity \\
\hline$(\mathbf{2}, \mathbf{2}, \mathbf{1})$ & {$[\sigma] \cdot[\tau]$} \\
\hline$(\mathbf{2}, \mathbf{1}, \mathbf{3})$ & {$[\sigma] \cdot[t]$} \\
\hline$(\mathbf{1}, \mathbf{2}, \mathbf{3})$ & {$[\tau] \cdot[t]$} \\
\hline$(\mathbf{2}, \mathbf{1}, \mathbf{1})$ & {$[\sigma] \cdot\left(-8 K_{B}-2[\sigma]-2[\tau]-3[t]\right)$} \\
\hline$(\mathbf{1}, \mathbf{2}, \mathbf{1})$ & {$[\tau] \cdot\left(-8 K_{B}-2[\sigma]-2[\tau]-3[t]\right)$} \\
\hline$(\mathbf{1}, \mathbf{1}, \mathbf{3})$ & {$[t] \cdot\left(-9 K_{B}-2[\sigma]-2[\tau]-3[t]\right)$} \\
\hline
\end{tabular}

We note that there are additional singular fibers of Kodaira type $I I I$ and $I V$ at the codimension two loci $\sigma=\left(\mathfrak{a}_{1}^{2}+4 \mathfrak{a}_{2} t\right)=0$ or $\tau=\left(\mathfrak{a}_{1}^{2}+4 \mathfrak{a}_{2} t\right)=0$ and $\mathfrak{a}_{1}=t=0$, respectively, that do not is support additional matter.

We readily check using the data in (B.4), (B.22) and the anomaly coefficients that all $6 \mathrm{D}$ anomalies are canceled.

\section{Map of $X_{n+1}$ to the quartic in $\mathrm{Bl}_{1} \mathbb{P}^{2}(1,1,2)$}

In this appendix, we argue that the unHiggsing of the F-theory model defined by $X_{n+1}$ can be interpreted as an unHiggsing of the two U(1)'s to two SU(2)'s on reducible divisors $X=A C$ and $Y=B C$. To this end, we use the natural presentation of [4] for a model with one $\mathrm{U}(1)$ as quartic in $\mathrm{Bl}_{1} \mathbb{P}^{2}(1,1,2)$, which will be non-generic with an additional non-toric rational section in the case of $X_{n+1}$. Most notably, due to this non-generic form the singularity on the common component $C$ enhances just to $I_{3}$, corresponding to an $\mathrm{SU}(3)$ gauge group. This is in contrast to the expected enhancement of two $I_{2}$ singularities to an $I_{4}$.

It was argued in [4] that every model with at least one rational section can be brought into the form of the quartic in $\mathrm{Bl}_{1} \mathbb{P}^{2}(1,1,2)$. In particular, this implies that there exists a birational map of the model (3.4) to the quartic. In the following, as an illustration we construct this birational map for the specialized model (3.8).

We recall from [4] that the quartic elliptic curve in $\mathrm{Bl}_{1} \mathrm{P}^{2}(1,1,2)$ with homogeneous coordinates $[U: V: W]$ (we set the coordinate of the exceptional divisor to one) reads

$$
W\left(W+b V^{2}\right)=\mathrm{U}\left(e_{0} U^{3}+e_{1} U^{2} V+e_{2} U V^{2}+e_{3} V^{3}\right) .
$$

It has a rational point $\tilde{Q}$ at $[U: V: W]=[0: 1:-b]$ and its zero point is at $[0: 1: 0]$. 
There are two possible presentations of the specialized model as the quartic (C.1), corresponding to the two possible choices which rational point in (3.8) is mapped to the rational point $\tilde{Q}$ in $\operatorname{Bl}_{1} \mathbb{P}^{2}(1,1,2)$. Here, we choose to map $Q$ in (3.8) to $\tilde{Q}$, noting that the quartic for the choice of $R \mapsto \tilde{Q}$ is obtained by exchanging $\left(a_{2}, b_{2}\right) \leftrightarrow\left(a_{3}, b_{3}\right)$.

One way to find the quartic is to construct sections in the bundles $\mathcal{O}(k(P+Q))$ for $k=1,2,3,4$. (This requires moving the point $R$ out of the plane $u=0$, which can be achieved by a variable transformation on (3.8).) Here, we construct the map indirectly by matching Weierstrass models. The Weierstrass model for the specialized model is given in appendix $\mathrm{A}$ for $a_{1}=1, b_{1}=0$. It has to agree with the Weierstrass form (2.3) upon appropriate identifications of the coefficients $b$ and $e_{i}, i=0,1,2,3$, with the coefficients in (3.8). Clearly, the system of equations resulting from this is under-determined. We have to specify in addition that the rational point $Q$ maps to the rational point $\tilde{Q}$ in (C.1). We recall that the Weierstrasss coordinates of $\tilde{Q} \operatorname{read}[4]$

$$
[x: y: z]=\left[e_{3}^{2}-\frac{2}{3} b^{2} e_{2}:-e_{3}^{3}+b^{2} e_{2} e_{3}-\frac{1}{2} b^{4} e_{1}: b\right] .
$$

Comparing to the Weierstrass coordinates (A.3) for $Q$ with $a_{1}=1$ and $b_{1}=0$ and demanding equality of the Weierstrass models, we obtain

$$
\begin{aligned}
b & =b_{2} \\
e_{0} & =\frac{1}{4}\left(b_{3}^{2}\left(s_{2}^{2}-4 s_{1} s_{3}\right)-2 a_{3} b_{3}\left(s_{2} s_{5}-2 s_{1} s_{6}\right)+a_{3}^{2}\left(s_{5}^{2}-4 s_{1} s_{8}\right)\right), \\
e_{1} & =\frac{1}{2} b_{3}\left(2 s_{3} s_{5}-s_{2} s_{6}+2 a_{2} b_{3} s_{1}\right)+a_{3}\left(s_{2} s_{8}-b_{2} b_{3} s_{1}-\frac{1}{2} s_{5} s_{6}\right), \\
e_{2} & =\frac{1}{4}\left(s_{6}^{2}-4 s_{3} s_{8}+2 b_{2}\left(a_{3} s_{5}+b_{3} s_{2}\right)-4 a_{2} b_{3} s_{5}\right), \\
e_{3} & =a_{2} s_{8}-\frac{1}{2} b_{2} s_{6} .
\end{aligned}
$$

The coordinates of the rational point $R$ in the quartic (C.1) are given by

$$
\begin{aligned}
{[U: V: W]=[} & b_{3}\left(a_{2} b_{3}-a_{3} b_{2}\right): a_{3}^{2} s_{8}-a_{3} b_{3} s_{6}+b_{3}^{2} s_{3}:-\frac{1}{2} b_{3}\left(b_{3}^{3}\left(a_{2}^{2} b_{3} s_{2}-a_{2} s_{3} s_{6}+2 b_{2} s_{3}^{2}\right)\right. \\
& +a_{3}^{2} b_{3}\left(b_{2}\left(s_{6}^{2}+2 s_{3} s_{8}+2 a_{2} b_{3} s_{5}\right)-3 a_{2} s_{6} s_{8}+b_{3} b_{2}^{2} s_{2}\right)-a_{3} b_{3}^{2}\left(a_{2}^{2} b_{3} s_{5}+3 b_{2} s_{3} s_{6}\right. \\
& \left.\left.\left.\quad-a_{2}\left(s_{6}^{2}+2 s_{3} s_{8}-2 b_{2} b_{3} s_{2}\right)\right)-a_{3}^{3}\left(b_{3} b_{2}^{2} s_{5}+b_{2} s_{6} s_{8}-2 a_{2} s_{8}^{2}\right)\right)\right] .
\end{aligned}
$$

We note that it is a non-toric rational point.

The coefficient $e_{3}$ is the locus of $\mathrm{SU}(2)$ enhancement if the section $\mathrm{U}(1)$ corresponding to the section $\hat{s}_{\tilde{Q}}$ is unHigssed. This unHiggsing is triggered by $b_{2} \rightarrow 0$ in which case we obtain $e_{3}=a_{2} s_{8}$ according to (C.3). Thus after unHiggsing we obtain two $\mathrm{SU}(2)$ 's on the two components $a_{2}=0$ and $s_{8}=0$. In contrast, if we set $b_{3} \rightarrow 0$ we unHiggs the U(1) associated to the non-toric section $\hat{s}_{R}$ as its coordinates in (C.4) then coincide with those of the zero point $[0: 1: 0]$. Furthermore, we observe from (C.3) that $e_{0} \sim a_{3}^{2}, e_{1} \sim a_{3}$, inducing an $I_{2}$ singularity at $a_{3}=0$. In addition, we obtain an $I_{2}$ at $s_{8}=0$ as can be seen 
from the discriminant. Thus we can interpret the tuning $b_{3} \rightarrow 0$ again as an unHiggsing to an $\mathrm{SU}(2)$ on a reducible divisor $e_{3}^{\prime}=a_{3} s_{8}$. In fact, $e_{3}^{\prime}$ is the coefficient in the second (C.1) we obtain by mapping $R \mapsto \tilde{Q}$.

We emphasize that in the simultaneous tuning $b_{2}, b_{3} \rightarrow 0$ the $I_{2}$ singularity on $s_{8}$ only enhances to an $I_{3}$. Thus, we confirm the picture of an $\mathrm{SU}(2)$ on two divisor $X=A C$, $Y=B C$ with $A=a_{2}, B=a_{3}$ and $C=s_{8}$ in the unHiggsed theory, as claimed.

Open Access. This article is distributed under the terms of the Creative Commons Attribution License (CC-BY 4.0), which permits any use, distribution and reproduction in any medium, provided the original author(s) and source are credited.

\section{References}

[1] C. Vafa, Evidence for F-theory, Nucl. Phys. B 469 (1996) 403 [hep-th/9602022] [INSPIRE].

[2] D.R. Morrison and C. Vafa, Compactifications of F-theory on Calabi-Yau threefolds. 1, Nucl. Phys. B 473 (1996) 74 [hep-th/9602114] [INSPIRE].

[3] D.R. Morrison and C. Vafa, Compactifications of F-theory on Calabi-Yau threefolds. 2, Nucl. Phys. B 476 (1996) 437 [hep-th/9603161] [INSPIRE].

[4] D.R. Morrison and D.S. Park, F-Theory and the Mordell-Weil Group of Elliptically-Fibered Calabi-Yau Threefolds, JHEP 10 (2012) 128 [arXiv:1208.2695] [INSPIRE].

[5] G. Aldazabal, A. Font, L.E. Ibáñez and A.M. Uranga, New branches of string compactifications and their F-theory duals, Nucl. Phys. B 492 (1997) 119 [hep-th/9607121] [INSPIRE].

[6] A. Klemm, P. Mayr and C. Vafa, BPS states of exceptional noncritical strings, hep-th/9607139 [INSPIRE].

[7] A. Klemm, M. Kreuzer, E. Riegler and E. Scheidegger, Topological string amplitudes, complete intersection Calabi-Yau spaces and threshold corrections, JHEP 05 (2005) 023 [hep-th/0410018] [INSPIRE].

[8] J. Borchmann, C. Mayrhofer, E. Palti and T. Weigand, Elliptic fibrations for $\mathrm{SU}(5) \times \mathrm{U}(1) \times \mathrm{U}(1)$ F-theory vacua, Phys. Rev. D 88 (2013) 046005 [arXiv: 1303.5054] [INSPIRE].

[9] M. Cvetič, D. Klevers and H. Piragua, F-Theory Compactifications with Multiple U(1)-Factors: Constructing Elliptic Fibrations with Rational sections, JHEP 06 (2013) 067 [arXiv: 1303.6970] [INSPIRE].

[10] M. Cvetič, A. Grassi, D. Klevers and H. Piragua, Chiral Four-Dimensional F-theory Compactifications With SU(5) and Multiple U(1)-Factors, JHEP 04 (2014) 010 [arXiv: 1306.3987] [INSPIRE].

[11] J. Borchmann, C. Mayrhofer, E. Palti and T. Weigand, SU(5) Tops with Multiple U(1)s in F-theory, Nucl. Phys. B 882 (2014) 1 [arXiv:1307.2902] [INSPIRE].

[12] M. Cvetič, D. Klevers and H. Piragua, F-Theory Compactifications with Multiple U(1)-Factors: Addendum, JHEP 12 (2013) 056 [arXiv:1307.6425] [INSPIRE]. 
[13] M. Cvetič, D. Klevers, H. Piragua and P. Song, Elliptic fibrations with rank three Mordell-Weil group: F-theory with $\mathrm{U}(1) \times \mathrm{U}(1) \times \mathrm{U}(1)$ gauge symmetry, JHEP 03 (2014) 021 [arXiv: 1310.0463] [INSPIRE].

[14] D.R. Morrison and W. Taylor, Sections, multisections and U(1) fields in F-theory, arXiv: 1404.1527 [INSPIRE].

[15] R. Donagi and M. Wijnholt, Model Building with F-theory, Adv. Theor. Math. Phys. 15 (2011) 1237 [arXiv: 0802.2969] [INSPIRE].

[16] C. Beasley, J.J. Heckman and C. Vafa, GUTs and Exceptional Branes in F-theory, I, JHEP 01 (2009) 058 [arXiv: 0802.3391] [INSPIRE].

[17] R. Donagi and M. Wijnholt, Breaking GUT Groups in F-theory, Adv. Theor. Math. Phys. 15 (2011) 1523 [arXiv: 0808.2223] [inSPIRE].

[18] J. Marsano, N. Saulina and S. Schäfer-Nameki, F-theory Compactifications for Supersymmetric GUTs, JHEP 08 (2009) 030 [arXiv:0904.3932] [INSPIRE].

[19] R. Blumenhagen, T.W. Grimm, B. Jurke and T. Weigand, Global F-theory GUTs, Nucl. Phys. B 829 (2010) 325 [arXiv: 0908.1784] [INSPIRE].

[20] T.W. Grimm, S. Krause and T. Weigand, F-Theory GUT Vacua on Compact Calabi-Yau Fourfolds, JHEP 07 (2010) 037 [arXiv: 0912.3524] [INSPIRE].

[21] J. Marsano, N. Saulina and S. Schäfer-Nameki, Compact F-theory GUTs with U(1) (PQ), JHEP 04 (2010) 095 [arXiv: 0912.0272] [INSPIRE].

[22] T.W. Grimm and T. Weigand, On Abelian Gauge Symmetries and Proton Decay in Global F-theory GUTs, Phys. Rev. D 82 (2010) 086009 [arXiv:1006.0226] [INSPIRE].

[23] E. Dudas and E. Palti, On hypercharge flux and exotics in F-theory GUTs, JHEP 09 (2010) 013 [arXiv: 1007.1297] [INSPIRE].

[24] M.J. Dolan, J. Marsano, N. Saulina and S. Schäfer-Nameki, F-theory GUTs with U(1) Symmetries: Generalities and Survey, Phys. Rev. D 84 (2011) 066008 [arXiv:1102.0290] [INSPIRE].

[25] S. Krause, C. Mayrhofer and T. Weigand, $G_{4}$ flux, chiral matter and singularity resolution in F-theory compactifications, Nucl. Phys. B 858 (2012) 1 [arXiv:1109.3454] [INSPIRE].

[26] S. Krause, C. Mayrhofer and T. Weigand, Gauge Fluxes in F-theory and Type IIB Orientifolds, JHEP 08 (2012) 119 [arXiv: 1202.3138] [INSPIRE].

[27] C. Mayrhofer, E. Palti and T. Weigand, U(1) symmetries in F-theory GUTs with multiple sections, JHEP 03 (2013) 098 [arXiv:1211.6742] [INSPIRE].

[28] V. Braun, T.W. Grimm and J. Keitel, Geometric Engineering in Toric F-theory and GUTs with U(1) Gauge Factors, JHEP 12 (2013) 069 [arXiv: 1306.0577] [INSPIRE].

[29] S. Krippendorf, D.K. Mayorga Pena, P.-K. Oehlmann and F. Ruehle, Rational F-theory GUTs without exotics, JHEP 07 (2014) 013 [arXiv:1401.5084] [INSPIRE].

[30] V. Braun, T.W. Grimm and J. Keitel, Complete Intersection Fibers in F-theory, JHEP 03 (2015) 125 [arXiv:1411.2615] [INSPIRE].

[31] L. Lin and T. Weigand, Towards the Standard Model in F-theory, Fortsch. Phys. 63 (2015) 55 [arXiv: 1406.6071] [INSPIRE]. 
[32] D. Klevers, D.K. Mayorga Pena, P.-K. Oehlmann, H. Piragua and J. Reuter, F-Theory on all Toric Hypersurface Fibrations and its Higgs Branches, JHEP 01 (2015) 142 [arXiv: 1408.4808] [INSPIRE].

[33] M. Cvetič, D. Klevers, D.K.M. Peña, P.-K. Oehlmann and J. Reuter, Three-Family Particle Physics Models from Global F-theory Compactifications, JHEP 08 (2015) 087 [arXiv: 1503.02068] [INSPIRE].

[34] M.B. Green, J.H. Schwarz and P.C. West, Anomaly Free Chiral Theories in Six-Dimensions, Nucl. Phys. B 254 (1985) 327 [INSPIRE].

[35] A. Sagnotti, A note on the Green-Schwarz mechanism in open string theories, Phys. Lett. B 294 (1992) 196 [hep-th/9210127] [INSPIRE].

[36] J. Erler, Anomaly cancellation in six-dimensions, J. Math. Phys. 35 (1994) 1819 [hep-th/9304104] [INSPIRE].

[37] D.S. Park and W. Taylor, Constraints on 6D Supergravity Theories with Abelian Gauge Symmetry, JHEP 01 (2012) 141 [arXiv:1110.5916] [INSPIRE].

[38] S. Krippendorf, S. Schäfer-Nameki and J.-M. Wong, Froggatt-Nielsen meets Mordell-Weil: A Phenomenological Survey of Global F-theory GUTs with U(1)s, JHEP 11 (2015) 008 [arXiv: 1507.05961] [INSPIRE].

[39] M.B. Green, J.H. Schwarz and E. Witten, Superstring theory: volume 2, Loop amplitudes, anomalies and phenomenology. Cambridge University Press, (2012).

[40] V. Kumar, D.R. Morrison and W. Taylor, Global aspects of the space of $6 D N=1$ supergravities, JHEP 11 (2010) 118 [arXiv:1008.1062] [INSPIRE].

[41] V. Kumar, D.S. Park and W. Taylor, 6D supergravity without tensor multiplets, JHEP 04 (2011) 080 [arXiv: 1011.0726] [INSPIRE].

[42] D.R. Morrison and W. Taylor, Matter and singularities, JHEP 01 (2012) 022 [arXiv: 1106.3563] [INSPIRE].

[43] V. Sadov, Generalized Green-Schwarz mechanism in F-theory, Phys. Lett. B 388 (1996) 45 [hep-th/9606008] [INSPIRE].

[44] P. Deligne, Courbes elliptiques: formulaire d'apres j. tate, in Modular functions of one variable, IV (Proceedings of the International Summer School, University of Antwerp, RUCA, July 17 - August 3, 1972), Lect. Notes Math. 476 (1975) 53.

[45] D.R. Morrison and W. Taylor, Classifying bases for $6 D$ F-theory models, Central Eur. J. Phys. 10 (2012) 1072 [arXiv:1201.1943] [InSPIRE].

[46] D.R. Morrison and W. Taylor, Non-Higgsable clusters for 4D F-theory models, JHEP 05 (2015) 080 [arXiv:1412.6112] [INSPIRE].

[47] J. Halverson and W. Taylor, $\mathbb{P}^{1}$-bundle bases and the prevalence of non-Higgsable structure in 4D F-theory models, JHEP 09 (2015) 086 [arXiv: 1506.03204] [INSPIRE].

[48] M. Esole and S.-T. Yau, Small resolutions of SU(5)-models in F-theory, Adv. Theor. Math. Phys. 17 (2013) 1195 [arXiv:1107.0733] [INSPIRE].

[49] J. Marsano and S. Schäfer-Nameki, Yukawas, G-flux and Spectral Covers from Resolved Calabi-Yau's, JHEP 11 (2011) 098 [arXiv:1108.1794] [INSPIRE].

[50] L.B. Anderson, I. García-Etxebarria, T.W. Grimm and J. Keitel, Physics of F-theory compactifications without section, JHEP 12 (2014) 156 [arXiv:1406.5180] [INSPIRE]. 
[51] P. Griffiths and J. Harris, Principles of algebraic geometry, John Wiley \& Sons, (2014).

[52] M. Cvetič, R. Donagi, D. Klevers, H. Piragua and M. Poretschkin, F-theory vacua with $\mathbb{Z}_{3}$ gauge symmetry, Nucl. Phys. B 898 (2015) 736 [arXiv:1502.06953] [INSPIRE].

[53] M. Esole, S.-H. Shao and S.-T. Yau, Singularities and Gauge Theory Phases II, arXiv: 1407.1867 [INSPIRE].

[54] A.P. Braun and S. Schäfer-Nameki, Box Graphs and Resolutions I, arXiv:1407.3520 [INSPIRE].

[55] D.S. Park, Anomaly Equations and Intersection Theory, JHEP 01 (2012) 093 [arXiv:1111.2351] [INSPIRE].

[56] C. Lawrie, S. Schäfer-Nameki and J.-M. Wong, F-theory and All Things Rational: Surveying U(1) Symmetries with Rational sections, JHEP 09 (2015) 144 [arXiv: 1504.05593] [INSPIRE].

[57] T. Shioda, On the Mordell-Weil lattices, Comment. Math. Univ. St. Paul 39 (1990) 211.

[58] J. Tate, Algorithm for determining the type of a singular fiber in an elliptic pencil, in Modular functions of one variable, IV (Proceedings of the International Summer School, University of Antwerp, RUCA, July 17 - August 3, 1972), Lect. Notes Math. 476 (1975) 33.

[59] W. Taylor, TASI Lectures on Supergravity and String Vacua in Various Dimensions, arXiv:1104.2051 [INSPIRE].

[60] A. Grassi and D.R. Morrison, Anomalies and the Euler characteristic of elliptic Calabi-Yau threefolds, Commun. Num. Theor. Phys. 6 (2012) 51 [arXiv:1109.0042] [InSPIRE].

[61] M. Bershadsky, K.A. Intriligator, S. Kachru, D.R. Morrison, V. Sadov and C. Vafa, Geometric singularities and enhanced gauge symmetries, Nucl. Phys. B 481 (1996) 215 [hep-th/9605200] [INSPIRE].

[62] V. Kumar and W. Taylor, String Universality in Six Dimensions, Adv. Theor. Math. Phys. 15 (2011) 325 [arXiv:0906 .0987] [INSPIRE].

[63] V. Kumar, D.R. Morrison and W. Taylor, Mapping $6 D N=1$ supergravities to F-theory, JHEP 02 (2010) 099 [arXiv: 0911.3393] [INSPIRE].

[64] N. Seiberg and W. Taylor, Charge Lattices and Consistency of 6D Supergravity, JHEP 06 (2011) 001 [arXiv:1103.0019] [INSPIRE].

[65] S. Katz, D.R. Morrison, S. Schäfer-Nameki and J. Sully, Tate's algorithm and F-theory, JHEP 08 (2011) 094 [arXiv: 1106.3854] [INSPIRE]. 